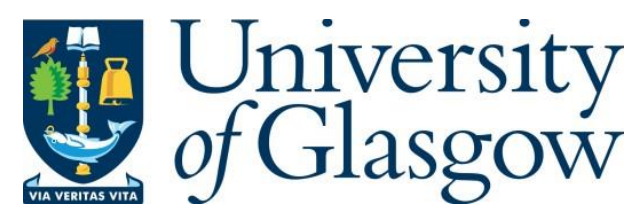

Bernreuther, W., Galler, P., Mellein, C., Si, Z.-G. and Uwer, P. (2016) Production of heavy Higgs bosons and decay into top quarks at the LHC. Physical Review D, 93(3), 034032.

There may be differences between this version and the published version. You are advised to consult the publisher's version if you wish to cite from it.

http://eprints.gla.ac.uk/154629/

Deposited on: 4 January 2018

Enlighten - Research publications by members of the University of Glasgow http://eprints.gla.ac.uk 
HU-EP-15/52

TTK-15-33

\title{
Production of heavy Higgs bosons and decay into top quarks at the LHC
}

\author{
W. Bernreuther ${ }^{a}, 1$, P. Galler ${ }^{b}$, C. Mellein $a \sqrt[3]{3}$ Z.-G. Si ${ }^{c} \sqrt[4]{ }$ and P. Uwer ${ }^{b} \sqrt[5]{5}$ \\ ${ }^{a}$ Institut für Theoretische Teilchenphysik und Kosmologie, RWTH Aachen University, \\ 52056 Aachen, Germany \\ ${ }^{b}$ Institut für Physik, Humboldt-Universität zu Berlin, 12489 Berlin, Germany \\ ${ }^{c}$ School of Physics, Shandong University, Jinan, Shandong 250100, China
}

\begin{abstract}
We investigate the production of heavy, neutral Higgs boson resonances and their decays to top-quark top-antiquark $(t \bar{t})$ pairs at the Large Hadron Collider (LHC) at next-to-leading order (NLO) in the strong coupling of quantum chromodynamics (QCD). The NLO corrections to heavy Higgs boson production and the Higgs-QCD interference are calculated in the large $m_{t}$ limit with an effective Kfactor rescaling. The nonresonant $t \bar{t}$ background is taken into account at NLO QCD including weakinteraction corrections. In order to consistently determine the total decay widths of the heavy Higgs bosons, we consider for definiteness the type-II two-Higgs-doublet extension of the standard model and choose three parameter scenarios that entail two heavy neutral Higgs bosons with masses above the $t \bar{t}$ threshold and unsuppressed Yukawa couplings to top quarks. For these three scenarios we compute, for the LHC operating at $13 \mathrm{TeV}$, the $t \bar{t}$ cross section and the distributions of the $t \bar{t}$ invariant mass, of the transverse top-quark momentum and rapidity, and of the cosine of the Collins-Soper angle with and without the two heavy Higgs resonances. For selected $M_{t \bar{t}}$ bins we estimate the significances for detecting a heavy Higgs signal in the $t \bar{t}$ dileptonic and lepton plus jets decay channels.
\end{abstract}

PACS number(s): 12.38.Bx, 12.60.Fr, 14.65.Ha, 14.80.Ec

Keywords: hadron collider physics, Higgs boson, top quark, QCD corrections, new physics

\footnotetext{
${ }^{1}$ breuther@physik.rwth-aachen.de

${ }^{2}$ galler@physik.hu-berlin.de

${ }^{3}$ mellein@physik.rwth-aachen.de

${ }^{4}$ zgsi@sdu.edu.cn

${ }^{5}$ uwer@physik.hu-berlin.de
} 


\section{Introduction}

The investigations of the production and decays of the $125 \mathrm{GeV}$ spin-zero resonance [1, 2] at the Large Hadron Collider (LHC) by the CMS [3] and ATLAS experiments [4] show that the properties of this particle are consistent with those of the Higgs boson of the standard model (SM). These results strongly support the Higgs mechanism of electroweak symmetry breaking, but do not exclude the possibility that additional Higgs bosons with masses below or around $1 \mathrm{TeV}$ exist. There are several well-known motivations for considering the existence of an extended Higgs sector (for reviews see, for instance, Refs. [5, 6]), and major experimental efforts at the LHC are being devoted to the search for additional spin-zero bosons. To date, these efforts include investigations which were aimed at tracing additional neutral Higgs bosons with significant couplings to the electroweak gauge bosons [7-11] or enhanced couplings (in comparison to respective SM Higgs couplings) to $b$ quarks [12, 13] and charged leptons [14,15]. These searches-negative so far-have an impact on parts of the parameter spaces of models with an extended Higgs sector but are, needless to say, by far not exhaustive.

One possibility, which is notoriously difficult to explore experimentally, is that one or several neutral Higgs bosons with masses above the top-antitop quark $(t \bar{t})$ production threshold exist, with significant Yukawa couplings to top quarks but suppressed couplings to the weak gauge bosons and to $d$-type quarks and charged leptons. Very likely, the resonant production of such a Higgs boson $\phi$ and its subsequent decay into $t \bar{t}$ at the LHC, $p p \rightarrow \phi \rightarrow t \bar{t} X$, does not show up as a resonance bump in the $t \bar{t}$ invariant mass distribution but as a distinctive peak-dip structure. This structure is caused by the interference of the signal and the nonresonant $t \bar{t}$ background amplitudes if the narrow-width approximation does not apply to $\phi$, which is very likely the case. This was first shown in Refs. [16, 17] for a scalar and pseudoscalar $\phi$, respectively, and in Refs. [18-20] also for a charge parity $(C P)$ mixture. In Refs. [18-20] effects of a heavy Higgs boson on top-quark spin observables were also investigated. More recently, the study of this $\phi$ decay channel was taken up again in Refs. [21-26]. All these investigations were performed at leading-order (LO) QCD.

First experimental attempts to search for heavy spin-zero states $\phi$ in the $t \bar{t}$ decay channel at the LHC were made by the CMS [27] and ATLAS [28] experiments. These investigations are difficult, in particular because the experimental resolution of the $t \bar{t}$ invariant mass is, in general, larger than the expected width of $\phi$, which hampers a resolution of the resonance region. On the theoretical side, a more precise description of the reaction $p p \rightarrow \phi \rightarrow t \bar{t} X$ is necessary. In this paper we investigate the resonant production of heavy neutral Higgs bosons $\phi$ and their decay into $t \bar{t}$ including an approximate calculation of the next-to-leading order QCD corrections. That is, the NLO Higgs production amplitudes are computed in the limit of $m_{t} \rightarrow \infty$. The interferences of the signal and nonresonant $t \bar{t}$ background amplitudes are also determined at NLO QCD. The nonresonant $t \bar{t}$ background is computed at NLO QCD and the mixed QCD-weak interaction corrections are included. We analyze how heavy Higgs bosons affect the $t \bar{t}$ cross section and several distributions. In particular, we determine the shape of the $t \bar{t}$ invariant mass distribution in the vicinity of a heavy Higgs resonance, which is of primary interest. We have analyzed the reaction at hand for arbitrary configurations of the $t$ and $\bar{t}$ spins, 
but we defer the investigation of heavy Higgs boson effects on $t \bar{t}$ spin correlations and on the $t$ and $\bar{t}$ polarization to a future publication.

The NLO QCD analysis of $p p \rightarrow \phi \rightarrow t \bar{t} X$ presented here may be applied to a number of SM extensions. Yet, in applications one has to fix the number of heavy Higgs bosons which can be resonantly produced in the $t \bar{t}$ channel. Other important ingredients in this analysis are the total widths of the Higgs bosons $\phi$ with mass larger than twice the top-quark mass. These widths must be computed in a concrete model, in order to maintain the unitarity of the $S$ matrix. For definiteness we have chosen to analyze the process at hand within the type-II two-Higgsdoublet extension (2HDM) of the standard model. This model, which may be realized with or without Higgs sector $C P$ violation, is among the simplest extensions of the SM. While interesting per se, it may also be considered a prototype of an extended Higgs/spin-zero sector. (For a recent review see, e.g., Ref. [6].) The spectrum of physical spin-zero particles of 2HDMs consists of three neutral Higgs bosons $\phi_{1}, \phi_{2}, \phi_{3}$ and a charged Higgs boson and its antiparticle, $H^{ \pm}$. We identify $\phi_{1}$ with the observed $125 \mathrm{GeV}$ resonance and assume that the masses $\phi_{2}$ and $\phi_{3}$ are larger than twice the top-quark mass. The charged Higgs boson is assumed to be heavy enough as to play no role in our analysis. The LHC results on the $125 \mathrm{GeV}$ Higgs resonance imply that the parameters of $2 \mathrm{HDMs}$ are constrained by the requirement of $\phi_{1}$ being SM-like. In addition we are interested in parameter scenarios where the top-quark Yukawa couplings of the heavy Higgs bosons $\phi_{2}, \phi_{3}$ are somewhat enhanced or at least as large as the SM top-quark Yukawa coupling - without simultaneous enhancement of the Yukawa couplings of $d$-type quarks and charged leptons - and where the couplings of $\phi_{2}, \phi_{3}$ to the weak gauge bosons are severely suppressed. This is possible in the type-II 2HDM. We define three such parameter scenarios which are in accord with experimental constraints-two scenarios with $C P$ conservation and one with $C P$ violation in the tree-level Higgs potential. For each of these parameter scenarios, we compute the Higgs boson contributions to the $t \bar{t}$ cross section and to several top-quark distributions, in particular the $t \bar{t}$ invariant mass distribution, for the LHC at $13 \mathrm{TeV}$. In the context of our type-II 2HDM parameter scenarios the NLO analysis is organized as follows: the nonresonant contribution of $\phi_{1}$ is part of the mixed QCD-weak interaction corrections to the nonresonant $t \bar{t}$ background. As to the amplitudes of $a b \rightarrow \phi_{j} \rightarrow t \bar{t} X$ ( $j=2,3$ ), where $a, b$ denote partons, their interference with the nonresonant $t \bar{t}$ amplitudes is taken into account. In the case of neutral Higgs-sector $C P$ violation, the $\phi_{2}$ and $\phi_{3}$ production amplitudes interfere also among each other but, as shown below, this contribution is very small for our parameter scenarios and can be neglected. The evaluation of our three parameter scenarios exemplifies the significance of the QCD corrections, but the effect of the two heavy Higgs bosons on the top-quark distributions is, overall, rather small. Nevertheless, statistically significant signals are possible. We propose to analyze existing and future LHC $t \bar{t}$ data by scanning the distribution of the $t \bar{t}$ invariant mass, $M_{t \bar{t}}$, with a sliding $M_{t \bar{t}}$ window of width $\sim 80 \mathrm{GeV}$.

The paper is organized as follows. In Sec. 2 we briefly recapitulate the main features of the type-II $2 \mathrm{HDM}$ with and without Higgs-sector $C P$ violation that are relevant for our analysis. In Sec. 3 we define three parameter scenarios which we use in the following: two sets associated with a $C P$-conserving and one set related to a $C P$-violating Higgs potential. We discuss that 
our scenarios are compatible with existing experimental constraints on the parameter space of the type-II 2HDM. Section 4 contains the setup of our calculations. For illustrative purposes we discuss first the resonant production of heavy Higgs bosons and their decay to $t \bar{t}$ and the interference with the nonresonant background at LO QCD. Then we outline how we compute distributions for this reaction including the Higgs-QCD interference at NLO QCD and we describe our K-factor approximation adapted from Ref. [29]. The nonresonant $t \bar{t}$ background is incorporated at NLO QCD including the weak-interaction corrections. Section 5 contains the results for $t \bar{t}$ production at the LHC (13 TeV) for the three parameter scenarios introduced in Sec. 3. First we compute the inclusive $t \bar{t}$ cross section and the $M_{t \bar{t}}$ distribution without and with the two heavy Higgs resonances. We estimate, for appropriately chosen $M_{t \bar{t}}$ intervals and for our three parameter scenarios, the significance with which a heavy Higgs signal could be detected in the $t \bar{t}$ dileptonic and lepton plus jets decay channels. Furthermore, we give the distributions of the rapidity and transverse momentum of the top quark and the distributions of the cosine of the Collins-Soper angle [30] for selected $M_{t \bar{t}}$ windows. Moreover we show that the negative experimental searches for heavy spin-zero resonances in the $t \bar{t}$ channel [27,28] at the LHC ( $8 \mathrm{TeV})$ do not exclude our parameter scenarios. We conclude in Sec.6. Appendix A contains a discussion of the complex mass scheme that we apply for the description of the heavy Higgs boson resonances. The cancellation of real and virtual nonfactorizable QCD corrections to $g g \rightarrow \phi_{j} \rightarrow t \bar{t}$ in the soft gluon approximation (SGA) is outlined in Appendix B.

\section{The type-II two-Higgs-doublet model}

As mentioned above, we choose the type-II two-Higgs-doublet model as an example of a SM extension which allows one to describe, in a consistent field-theoretic and phenomenologically viable way, both the $125 \mathrm{GeV}$ Higgs resonance and additional neutral heavy Higgs bosons. In this section we shortly describe the salient features of several variants of this model which are relevant for our analysis.

In 2 HDMs the SM field content is extended by an additional Higgs doublet. Both Higgs doublets $\Phi_{1}$ and $\Phi_{2}$ transform under $\mathrm{SU}(3)_{c} \times \mathrm{SU}(2)_{L} \times \mathrm{U}(1)_{Y}$ as $(1,2,1 / 2)$.

The type-II model is defined by its Yukawa coupling structure: the doublet $\Phi_{1}$ is coupled to right-chiral down-type quarks and charged leptons, while $\Phi_{2}$ is coupled to right-chiral uptype quarks only. The type-II model belongs to those $2 \mathrm{HDMs}$ where, by construction, flavorchanging neutral currents are absent at tree level. The most general Hermitian, gauge invariant and renormalizable $2 \mathrm{HDM}$ potential reads (see, for instance, [6]):

$$
\begin{aligned}
V\left(\Phi_{1}, \Phi_{2}\right)= & -\mu_{1}^{2}\left|\Phi_{1}\right|^{2}-\mu_{2}^{2}\left|\Phi_{2}\right|^{2}-\left(\mu_{3}^{2} \Phi_{1}^{\dagger} \Phi_{2}+\text { h.c. }\right) \\
& +\frac{\lambda_{1}}{2}\left|\Phi_{1}\right|^{4}+\frac{\lambda_{2}}{2}\left|\Phi_{2}\right|^{4}+\lambda_{3}\left|\Phi_{1}\right|^{2}\left|\Phi_{2}\right|^{2}+\lambda_{4}\left|\Phi_{1}^{\dagger} \Phi_{2}\right|^{2} \\
& +\left[\frac{\lambda_{5}}{2}\left(\Phi_{1}^{\dagger} \Phi_{2}\right)^{2}+\lambda_{6}\left(\Phi_{1}^{\dagger} \Phi_{1}\right)\left(\Phi_{1}^{\dagger} \Phi_{2}\right)+\lambda_{7}\left(\Phi_{2}^{\dagger} \Phi_{2}\right)\left(\Phi_{1}^{\dagger} \Phi_{2}\right)+\text { h.c. }\right],
\end{aligned}
$$

with real $\mu_{1}^{2}, \mu_{2}^{2}, \lambda_{1,2,3,4}$ and complex $\mu_{3}^{2}, \lambda_{5,6,7}$, adding up to 14 independent real parameters. 
Three of the 14 parameters can be eliminated by a change of basis, $\Phi_{i}^{\prime}=U_{i j} \Phi_{j}$, where $U$ is a unitary $2 \times 2$ matrix.

After electroweak gauge symmetry breaking, using the unitary gauge the two-Higgs doublets take the form

$$
\begin{aligned}
& \Phi_{1}=\left(-H^{+} \sin \beta, \frac{1}{\sqrt{2}}\left(v_{1}+\varphi_{1}-i A \sin \beta\right)\right)^{T}, \\
& \Phi_{2}=e^{i \xi}\left(H^{+} \cos \beta, \frac{1}{\sqrt{2}}\left(v_{2}+\varphi_{2}+i A \cos \beta\right)\right)^{T},
\end{aligned}
$$

where $\tan \beta=v_{2} / v_{1}$ is the ratio of the vacuum expectation values of the two-Higgs-doublet fields with $v=\sqrt{v_{1}^{2}+v_{2}^{2}}=246 \mathrm{GeV}$. By a field redefinition the phase $\xi$ can be put to zero without loss of generality. The field $H^{+}$describes the physical charged Higgs boson of the model, while $\varphi_{1,2}$ and $A$ are the physical neutral $C P$-even and $C P$-odd field degrees of freedom. The Higgs potential (1) leads to mixing among these neutral fields. We denote the neutral Higgs fields and the associated Higgs particles of definite mass by $\phi_{j}, j=1,2,3$. They are related to $\varphi_{1,2}$ and $A$ by an orthogonal transformation, $\left(\phi_{1}, \phi_{2}, \phi_{3}\right)^{T}=R\left(\varphi_{1}, \varphi_{2}, A\right)^{T}$. We parametrize the orthogonal matrix $R$ as follows:

$$
R=\left(\begin{array}{ccc}
c_{1} c_{2} & c_{2} s_{1} & s_{2} \\
-c_{1} s_{2} s_{3}-c_{3} s_{1} & c_{1} c_{3}-s_{1} s_{2} s_{3} & c_{2} s_{3} \\
-c_{1} c_{3} s_{2}+s_{1} s_{3} & -c_{1} s_{3}-c_{3} s_{1} s_{2} & c_{2} c_{3}
\end{array}\right)
$$

where $c_{i}$ and $s_{i}(i=1,2,3)$ are the cosines and sines of the mixing angles $\alpha_{i}$. Due to the symmetries of $R[31]$ one can restrict the range of the angles to $-\pi / 2 \leq \alpha_{i}<\pi / 2$.

The Higgs potential in its general form (1) violates the $C P$ symmetry and, as a consequence, the $C P$-even and $C P$-odd Higgs fields mix and the mass eigenstates $\phi_{j}(j=1,2,3)$ do not have a definite $C P$. In this general $C P$-violating version of the type-II $2 \mathrm{HDM}$, the Higgs potential has, as mentioned above, 11 independent parameters.1. For phenomenological studies it is useful to choose as independent parameters a different set, in terms of which the parameters of the potential can be expressed. This set includes the following nine parameters which we call phenomenological parameters:

$$
m_{1}, m_{2}, m_{3}, m_{+}, \alpha_{1}, \alpha_{2}, \alpha_{3}, \tan \beta, v,
$$

where $m_{j}(j=1,2,3)$ are the masses of the three neutral Higgs bosons and $m_{+}$is the mass of $H^{+}$. The value of $v$ is of course fixed by experiment.

In order to motivate the type-II 2HDM construction by a symmetry argument, one often imposes a $Z_{2}$ symmetry onto the model which may be softly broken by the Higgs potential. This approximate symmetry implies that the hard $Z_{2}$ symmetry-breaking terms in (1) are absent, $\lambda_{6}=\lambda_{7}=0$. This variant of the model still allows for neutral Higgs sector $C P$ violation [33], i.e., for the mixing of $\varphi_{1,2}$ and $A$, if $\operatorname{Im}\left(\lambda_{5} / \mu_{3}^{4}\right) \neq 0$. One can show [34] that in this case the

\footnotetext{
${ }^{1}$ For a general discussion, see Ref. [32].
} 
number of independent phenomenological parameters listed in (4) is reduced by one. In the model with approximate $Z_{2}$ symmetry where $\operatorname{Im} \lambda_{5} \neq 0$, one can, for instance, express the mass $m_{3}$ of the neutral Higgs boson $\phi_{3}$ in terms of $m_{1}, m_{2}, \tan \beta$, and the Higgs mixing angles $\alpha_{i}(i=1,2,3)$. In our applications below, where we consider also the case of Higgs sector $C P$ violation, we however refer to the more general case where (4) is an independent parameter set, in order to avoid a theoretical bias.

The type-II $2 \mathrm{HDM}$ where the Higgs potential conserves $C P$ is a special case and is also of great phenomenological interest. In this case only $\varphi_{1}$ and $\varphi_{2}$ mix. This is described by the mixing angle $\alpha_{1}$, while $\alpha_{2}=\alpha_{3}=0$. The matrix $R$ is now block diagonal with $R_{13}=R_{23}=$ $R_{31}=R_{32}=0$ and $R_{33}=1$. The two $C P$-even and the $C P$-odd neutral mass eigenstates are often conventionally denoted by $\phi_{1}=h, \phi_{2}=H$, and $\phi_{3}=A$. The type-II 2HDM with $C P$ conserving Higgs potential may be motivated by assuming an approximate $Z_{2}$ symmetry. Then the potential has eight independent parameters, but they are reduced by one by the condition that the ground state of the model does not break $C P$ spontaneously. Thus, one may choose as independent parameters the following set:

$$
m_{1}, m_{2}, m_{3}, m_{+}, \alpha_{1}, \tan \beta, v .
$$

In the following we identify the $125 \mathrm{GeV}$ Higgs resonance with the Higgs boson $\phi_{1}$ and assume that the masses of the two other neutral states $\phi_{2,3}$ lie above the $t \bar{t}$ threshold. In addition, we assume that the mass of the charged Higgs boson $H^{+}$is of the order of the masses of $\phi_{2,3}$, so that the two-body decays $\phi_{2,3} \rightarrow W^{ \pm} H^{\mp}$ are kinematically excluded. Fits to LHC data [3, 4] imply that the couplings of the $125 \mathrm{GeV}$ Higgs boson to the third-generation fermions and gauge bosons are not too different from those of the SM Higgs boson. In the context of the $C P$-conserving $2 \mathrm{HDM}$ this means that the model is close to the so-called alignment limit ${ }^{2}$ $\beta=\alpha_{1}$. Yet, at present the uncertainties on the measured couplings of the $125 \mathrm{GeV}$ resonance are still rather large, $\sim 20 \%-30 \%$. In particular it is not yet experimentally shown that the $125 \mathrm{GeV}$ resonance is a pure $C P$-even state - a pseudoscalar admixture is still a possibility. Nevertheless, in the scenarios which we define in the next section and apply subsequently we shall choose parameters in or close to the alignment limit.

For our analysis below we need the interactions of the neutral Higgs bosons with fermions and weak gauge bosons, and their interactions among each other. We parametrize the couplings of the $\phi_{j}$ to the quarks and charged leptons $f=q, \ell$ and to weak gauge boson pairs in the type-II model with Higgs sector $C P$ violation as follows:

$$
\mathcal{L}_{1}=-\frac{m_{f}}{v}\left(a_{j f} \bar{f} f-b_{j f} \bar{f} i \gamma_{5} f\right) \phi_{j}+f_{j V V} \phi_{j}\left(\frac{2 m_{W}^{2}}{v} W_{\mu}^{-} W^{+\mu}+\frac{m_{Z}^{2}}{v} Z_{\mu} Z^{\mu}\right),
$$

where a sum over $f$ and $j=1,2,3$ is implicit. The reduced scalar and pseudoscalar Yukawa couplings $a_{j f}$ and $b_{j f}$ and the reduced couplings $f_{j V V}$ depend on the values of $\tan \beta$ and on

\footnotetext{
${ }^{2}$ Often the parametrization of the mixing matrix $R$ used in the literature is different from (3). For instance, in the parametrizations of [34,35], $\alpha=\alpha_{1}-\pi / 2$; i.e., the alignment limit is then given by $\beta-\alpha=\pi / 2$.
} 
the elements $R_{i j}$ of the Higgs mixing matrix (3) and are listed in Table 1 . The couplings $f_{j V V}$ satisfy the sum rule

$$
\sum_{j=1}^{3} f_{j V V}^{2}=1 .
$$

The interaction of neutral Higgs bosons $\phi_{j}, \phi_{l}(j \neq l)$ with the $Z$ boson is given by

$$
\mathcal{L}_{2}=-\frac{g_{W}}{2 \cos \theta_{W}} f_{Z j l} Z^{\mu} \phi_{j} \stackrel{\leftrightarrow}{\partial_{\mu}} \phi_{l}
$$

where $g_{W}$ is the $\mathrm{SU}(2)_{L}$ gauge coupling and $\theta_{W}$ denotes the weak mixing angle. For computing the rates of the decays $\phi_{j} \rightarrow Z \phi_{1}(j=2,3)$, we need the couplings $f_{Z j 1}$. They are given by

$$
f_{Z j 1}=\left(-\sin \beta R_{11}+\cos \beta R_{12}\right) R_{j 3}-\left(-\sin \beta R_{j 1}+\cos \beta R_{j 2}\right) R_{13} .
$$

Moreover, for computing the decays $\phi_{j} \rightarrow 2 \phi_{1}(j=2,3)$ the trilinear neutral Higgs couplings are required, $\mathcal{L}_{3}=g_{i j l} \phi_{i} \phi_{j} \phi_{l}$. In the $C P$-violating type-II $2 \mathrm{HDM}$ the couplings $g_{i j l}$ have a quite complicated form. They were derived in terms of the potential parameters in [35] and in terms of the phenomenological parameters in [36].

Table 1: Reduced couplings to quarks, leptons and gauge bosons of the neutral Higgs bosons $\phi_{j}$ of the type-II 2HDM in the parametrization (6). The labels $t, b$, and $\tau$ are place holders for $u$-type, $d$-type quarks, and charged leptons.

$$
\begin{array}{cccc|c}
a_{j t} & a_{j b}=a_{j \tau} & b_{j t} & b_{j b}=b_{j \tau} & f_{j V V} \\
\hline \hline R_{j 2} / \sin \beta & R_{j 1} / \cos \beta & R_{j 3} \cot \beta & R_{j 3} \tan \beta & \cos \beta R_{j 1}+\sin \beta R_{j 2}
\end{array}
$$

\section{Three type-II $2 \mathrm{HDM}$ scenarios}

In this section we define three phenomenologically viable parameter sets for the type-II 2HDM, two for the $C P$-conserving and one for the $C P$-violating variant, which we use in the next sections. We compute for these parameter sets the total widths of the heavy neutral Higgs bosons $\phi_{2}, \phi_{3}$, including QCD corrections. As already mentioned above, in all three cases we identify the $125 \mathrm{GeV}$ Higgs boson with $\phi_{1}$ and choose the parameters such that its couplings to fermions and gauge bosons are SM-like. For the parameter sets below, the total width $\Gamma_{1}$ of $\phi_{1}$ is of the order of $4 \mathrm{MeV}$. This width plays no role in the computations of Secs. 4-5. For the masses of the two other neutral Higgs bosons we assume $m_{2}, m_{3}>2 m_{t}$. The resonant production of $\phi_{2}, \phi_{3}$ has a chance of being experimentally detectable in the $t \bar{t}$ decay channel only if the Yukawa couplings are not suppressed in comparison with the SM top-Yukawa coupling. Therefore we choose $\tan \beta=0.7$ for all three scenarios below. Experimental constraints from 
$B$ physics require that for $\tan \beta<1$ the mass $m_{+}$of the charged Higgs boson is large. In order to satisfy these constraints [37-39] we assume that $m_{+}>720 \mathrm{GeV}$. This implies for the parameter scenarios below that the decays $\phi_{2,3} \rightarrow W^{ \pm} H^{\mp}$ cannot occur.

We determine the total widths of $\phi_{2}$ and $\phi_{3}$ by computing the sum of the largest two-body decay rates. The QCD corrections to the decays $\phi_{j} \rightarrow q \bar{q}$ and $\phi_{j} \rightarrow g g$ may be computed with the computer program HDECAY [40] or 2HDMC [41]. Both codes apply only to $C P$-conserving $2 \mathrm{HDM}$ but can be easily adapted to $C P$-violating scenarios. Since both programs use the renormalization scale $\mu=m_{\phi}$, which is not suited for our applications (see below), we use the expressions for the partial decay rates to quarks and to gluons including QCD corrections from Refs. [42,43] and Ref. [44], respectively. Expressions for the other decay rates (including the $C P$-violating case) can also be found in the literature, see e.g. Ref. [35]. Where possible we checked our results with 2HDMC. In these computations we use the top-quark mass $m_{t}$ in the on-shell scheme and put $m_{t}=173.34 \mathrm{GeV}$. Moreover, in order to be consistent with our computations of Secs. 4-5 we consider the following values and variations of the renormalization scale $\mu$ :

$$
\frac{\mu_{0}}{2} \leq \mu \leq 2 \mu_{0}, \quad \mu_{0}=\frac{m_{2}+m_{3}}{4} .
$$

Electroweak corrections to the decays $\phi_{j} \rightarrow f \bar{f}[45]$ and $\phi_{j} \rightarrow V V[46]$ are sizeable, especially for heavy Higgs bosons. Because we consider parameter scenarios where the $\phi_{j} V V$ couplings are suppressed for $j=2,3$, these corrections to $\phi_{j} \rightarrow V V$ will not affect the total widths $\Gamma_{2,3}$ very much. The electroweak corrections to $\phi_{j} \rightarrow t \bar{t}$ can be as large as the QCD corrections. Because we analyze in Sec. 4 resonant hadronic heavy Higgs boson production and decay to $t \bar{t}$ only to lowest order in the non-QCD couplings, we omit the contributions of the electroweak corrections to $\Gamma_{2,3}$.

\subsection{Scenario 1}

We consider the type-II model with a $C P$-conserving Higgs potential. We choose

$$
\tan \beta=0.7, \quad \alpha_{1}=\beta=0.611, \quad \alpha_{2}=\alpha_{3}=0,
$$

and choose the following Higgs boson masses, with $\phi_{2}$ and $\phi_{3}$ being nearly mass degenerate:

$$
m_{1}=125 \mathrm{GeV}, \quad m_{2}=550 \mathrm{GeV}, \quad m_{3}=510 \mathrm{GeV}, \quad m_{+}>720 \mathrm{GeV} .
$$

That is, $\phi_{1}$ and $\phi_{2}$ are chosen to be pure scalars while $\phi_{3}$ is a pseudoscalar Higgs boson, cf. Table 2. The reduced Yukawa couplings and couplings to the weak gauge bosons, which result from (11) and the formulas of Table 1, are given in Table 2. Recall that a pseudoscalar Higgs boson has no tree-level couplings to $W^{+} W^{-}$and $Z Z\left(f_{3 V V}=0\right)$. The choice $\alpha_{1}=\beta$ puts the model into the alignment limit. Therefore we have also $f_{2 V V}=0$.

We compute the largest two-body decay rates of the scalar $\phi_{2}$ and the pseudoscalar $\phi_{3}$ and determine their total widths by adding up these rates. The leading-order decay-rate formulas 
Table 2: Values of the reduced couplings to the third-generation fermions and gauge bosons of the neutral Higgs bosons $\phi_{j}$ for the parameter set (11) which is used in scenarios 1 and 2 .

\begin{tabular}{c|cccc|c} 
& $a_{j t}$ & $a_{j b}=a_{j \tau}$ & $b_{j t}$ & $b_{j b}=b_{j \tau}$ & $f_{j V V}$ \\
\hline \hline$\phi_{1}$ & 1 & 1 & 0 & 0 & 1 \\
$\phi_{2}$ & 1.429 & -0.700 & 0 & 0 & 0 \\
$\phi_{3}$ & 0 & 0 & 1.429 & 0.700 & 0 \\
\hline
\end{tabular}

Table 3: Dominant partial decay widths and the total widths of the two heavy, neutral Higgs bosons $\phi_{2}$ and $\phi_{3}$ in our $C P$-conserving $2 \mathrm{HDM}$ scenario 1, defined in (11)-(12). The super- and subscripts denote the shift due to the scale variations $(10)$.

\begin{tabular}{l|ll} 
& $\Gamma_{2}[\mathrm{GeV}]$ & $\Gamma_{3}[\mathrm{GeV}]$ \\
\hline$\phi_{i} \rightarrow t \bar{t}$ & $34.48_{-0.28}^{+0.33}$ & $49.15_{-0.32}^{+0.38}$ \\
$\phi_{i} \rightarrow g g$ & $0.08_{-0.01}^{+0.01}$ & $0.13_{-0.02}^{+0.02}$ \\
\hline Total & $34.56_{-0.28}^{+0.34}$ & $49.28_{-0.34}^{+0.40}$
\end{tabular}

for $\phi_{j} \rightarrow f \bar{f}, V V, Z \phi_{l}, 2 \phi_{l}, g g$, where $V=W, Z$, are well-known and are not reproduced here (see, e.g., [41]). The relevant tree-level couplings are given in Sec. 2. The trilinear neutral Higgs couplings are taken from [35, 36] and we use the QCD corrections to $\phi_{2,3} \rightarrow t \bar{t}, g g$ given in Ref. [42-44]. The results are listed in Table 3. The uncertainties result from varying the renormalization scale according to $(10)$. The partial decay widths of $\phi_{j} \rightarrow f \bar{f}(f \neq t), \phi_{j} \rightarrow \gamma \gamma$, and $\phi_{j} \rightarrow Z \gamma$ are a few $10^{-3} \mathrm{GeV}$ or smaller and are neglected in the total widths $\Gamma_{2}, \Gamma_{3}$. In particular, the decay rate to $b \bar{b}$ is small because the Yukawa couplings to down-type quarks are not enhanced (Table 2) due to our choice of $\tan \beta<1$. Moreover, to lowest order in the non-QCD couplings the partial decay rates for $\phi_{i} \rightarrow V V, \phi_{i} \rightarrow \phi_{1} Z$, and $\phi_{i} \rightarrow \phi_{1} \phi_{1}$ are zero.

\subsection{Scenario 2}

We choose again a $C P$-conserving neutral Higgs sector scenario with the same set of angles, Eq. (11), as in scenario 1. Here we assume that the pseudoscalar $\phi_{3}$ is significantly heavier than the scalar $\phi_{2}$. We choose

$$
m_{1}=125 \mathrm{GeV}, \quad m_{2}=550 \mathrm{GeV}, \quad m_{3}=700 \mathrm{GeV}, \quad m_{+}>720 \mathrm{GeV} .
$$

The values of the reduced couplings of $\phi_{2}, \phi_{3}$ to the third-generation fermions and gauge bosons given in Table 2 apply also here. The results for the most important two-body decay widths and the total widths of $\phi_{2}, \phi_{3}$ are given in Table 4. To lowest order in the non-QCD couplings the partial decay rates for $\phi_{i} \rightarrow V V, \phi_{i} \rightarrow \phi_{1} Z, \phi_{i} \rightarrow \phi_{1} \phi_{1}$, and $\phi_{3} \rightarrow \phi_{1} \phi_{2}$ are zero. 
Notice that the decay widths for $\phi_{2} \rightarrow V V, \phi_{2} \rightarrow \phi_{1} \phi_{1}$ and $\phi_{3} \rightarrow Z \phi_{1}$ vanish in scenarios 1 and 2, respectively, because we have chosen the exact alignment limit $\alpha_{1}=\beta$. The more one moves away from this limit, the larger the contributions of these modes will be to the total decay widths.

Table 4: Dominant partial decay widths and total widths of the two heavy, neutral Higgs bosons $\phi_{2}$ and $\phi_{3}$ in our $C P$-conserving 2HDM scenario 2, defined in (11) and (13). The super- and subscripts denote the shift due to the scale variations 10$]$.

\begin{tabular}{l|ll} 
& $\Gamma_{2}[\mathrm{GeV}]$ & $\Gamma_{3}[\mathrm{GeV}]$ \\
\hline$\phi_{i} \rightarrow t \bar{t}$ & $34.41_{-0.26}^{+0.32}$ & $71.97_{+0.13}^{-0.15}$ \\
$\phi_{i} \rightarrow \phi_{2} Z$ & 0 & 3.14 \\
$\phi_{i} \rightarrow g g$ & $0.08_{-0.01}^{+0.01}$ & $0.17_{-0.01}^{+0.01}$ \\
\hline Total & $34.49_{-0.27}^{+0.33}$ & $75.28_{+0.11}^{-0.14}$
\end{tabular}

\subsection{Scenario 3}

We consider a type-II model with a $C P$-violating Higgs potential (without approximate $Z_{2}$ symmetry) such that (4) forms a set of independent parameters. For $\tan \beta$ and the Higgs mixing angles we choose

$$
\tan \beta=0.7, \quad \alpha_{1}=\beta, \quad \alpha_{2}=\frac{\pi}{15}, \quad \alpha_{3}=\frac{\pi}{4} .
$$

We set the Higgs boson masses to the values

$$
m_{1}=125 \mathrm{GeV}, \quad m_{2}=500 \mathrm{GeV}, \quad m_{3}=800 \mathrm{GeV}, \quad m_{+}>720 \mathrm{GeV} .
$$

Using Table 1 and the parameters (14) we get the values of the reduced Yukawa couplings and couplings to the weak gauge bosons listed in Table 5. As this table shows, in this scenario the three neutral Higgs bosons $\phi_{1,2,3}$ are $C P$ mixtures.

Table 5: Values of the reduced couplings to the third-generation fermions and gauge bosons of the neutral Higgs bosons $\phi_{j}$ for the parameter set (14) of scenario 3.

\begin{tabular}{c|cccc|c} 
& $a_{j t}$ & $a_{j b}=a_{j \tau}$ & $b_{j t}$ & $b_{j b}=b_{j \tau}$ & $f_{j V V}$ \\
\hline \hline$\phi_{1}$ & 0.978 & 0.978 & 0.297 & 0.146 & 0.978 \\
$\phi_{2}$ & 0.863 & -0.642 & 0.988 & 0.484 & -0.147 \\
$\phi_{3}$ & -1.157 & 0.348 & 0.988 & 0.484 & -0.147 \\
\hline
\end{tabular}

The results for the most important two-body decay widths and the total widths of $\phi_{2}, \phi_{3}$ are given in Table 6. In this scenario without approximate $Z_{2}$ symmetry we have to specify also 
Table 6: Dominant partial decay widths and total widths of the two heavy, neutral Higgs bosons $\phi_{2}$ and $\phi_{3}$ in our $C P$-violating 2HDM scenario 3, defined in (14)-(15). The super- and subscripts denote the shift due to the scale variations (10).

\begin{tabular}{l|ll} 
& $\Gamma_{2}[\mathrm{GeV}]$ & $\Gamma_{3}[\mathrm{GeV}]$ \\
\hline$\phi_{i} \rightarrow t \bar{t}$ & $32.31_{-0.26}^{+0.31}$ & $85.05_{+0.25}^{-0.30}$ \\
$\phi_{i} \rightarrow V V$ & 1.12 & 5.11 \\
$\phi_{i} \rightarrow \phi_{1} Z$ & 0.65 & 3.24 \\
$\phi_{i} \rightarrow \phi_{2} Z$ & 0 & 31.28 \\
$\phi_{i} \rightarrow \phi_{1} \phi_{1}$ & 2.38 & 3.00 \\
$\phi_{i} \rightarrow \phi_{1} \phi_{2}$ & 0 & 0.31 \\
$\phi_{i} \rightarrow g g$ & $0.08_{-0.01}^{+0.01}$ & $0.17_{-0.01}^{+0.01}$ \\
\hline Total & $36.55_{-0.27}^{+0.32}$ & $128.16_{+0.24}^{-0.29}$
\end{tabular}

the values of the complex parameters $\lambda_{6}, \lambda_{7}$ in the $2 \mathrm{HDM}$ potential. These parameters only enter the calculation of the decay widths which involve trilinear Higgs couplings, i.e. only the decays $\phi_{i} \rightarrow \phi_{1} \phi_{1}$ and $\phi_{i} \rightarrow \phi_{1} \phi_{2}$ listed in Table 6. We choose $\operatorname{Re} \lambda_{6}=0, \operatorname{Im} \lambda_{6}=-3.677$ and $\lambda_{7}=0$.

\subsection{Experimental constraints}

The Higgs couplings and masses chosen in the three scenarios above are compatible with existing experimental constraints. As to the couplings of $\phi_{1}$, in scenarios 1 and 2 the couplings of $\phi_{1}$ are identical to those of the SM Higgs boson. In the $C P$-violating scenario $\phi_{1}$ has a pseudoscalar component. Nevertheless, also in this scenario the couplings of $\phi_{1}$ given in Table 5 lie within the respective coupling ranges of the $125 \mathrm{GeV}$ boson determined by the CMS [3] and ATLAS [4] experiments, which allow 20\%-30\% deviations from the respective SM Higgs couplings. Because in our scenarios the tree-level couplings of $\phi_{2}, \phi_{3}$ to $b$ quarks, $\tau$ leptons, weak gauge bosons, and to $\phi_{1}$ are very small or even zero, the negative searches, so far, at the LHC for nonstandard heavy resonances decaying to $W^{+} W^{-}[11], Z Z$ [7, 11], $Z \phi_{1}[8,10], \phi_{1} \phi_{1}[9,13], \tau^{-} \tau^{+}[14,15], b \bar{b}$ [12] do not exclude the above scenarios. We comment in Sec. 5] on the CMS [27] and ATLAS [28] searches in the $t \bar{t}$ decay channel.

The nonobservation of deviations from the SM predictions in B-physics data places limits on $\tan \beta$ and on the mass $m_{+}$of the charged Higgs boson. These parameters control the contribution of $H^{ \pm}$to loop-induced observables like the rare decays $B \rightarrow X_{s}+\gamma$ and neutral $B_{d}^{0}-\bar{B}_{d}^{0}$ mixing. In general the loop effects of charged Higgs bosons decrease with increasing mass $m_{+}$ and vice versa. Our choice $\tan \beta=0.7$ and $m_{+}>720 \mathrm{GeV}$ in our three $2 \mathrm{HDM}$ scenarios falls into the allowed parameter range which is determined by comparing experimental data with 2HDM predictions [37-39]. 
The allowed parameters of 2HDM are also restricted by the experimental values of the oblique electroweak parameters. The general expressions for the oblique parameters $S, T$, and $U$ in $2 \mathrm{HDM}$ and discussion of resulting constraints can be found in [6, 47.-49]. As we have chosen $\phi_{1}$ to be SM-like, $\phi_{2}, \phi_{3}$ and $H^{ \pm}$to be heavy, and the mixing angles such that the couplings of $\phi_{2}, \phi_{3}$ to the weak gauge bosons are suppressed, our scenarios are compatible with the constraints from electroweak precision measurements.

The experimental upper limits on the electric dipole moments of the neutron [50] and the electron [51] put limits on the angles associated with Higgs sector $C P$-violation in 2DHM. Our choice of Higgs mixing angles (14) in the $C P$-violating scenario 3 which implies the Higgs couplings given Table 5 and our choice of Higgs boson masses (15) lies within the allowed parameter range of $C P$-violating $2 \mathrm{HDM}$ without approximate $Z_{2}$ symmetry as derived, for instance, in [35].

\section{Theoretical predictions beyond the QCD Born approximation}

As mentioned in the introduction the aim of this article is to study, within the type-II twoHiggs-doublet model, the resonant production of heavy neutral Higgs bosons and their decay into $t \bar{t}$,

$$
p p \rightarrow \phi_{2,3} \rightarrow t \bar{t} X
$$

including the nonresonant $\mathrm{SM} t \bar{t}$ background

$$
p p \rightarrow t \bar{t} X
$$

and the interference of the resonant and nonresonant amplitudes. Specifically, we consider the three scenarios introduced in Sec. 3 and derive predictions for the cross section and distributions including all relevant NLO QCD corrections.

\subsection{Setup and leading-order results}

In order to set up our conventions and introduce some abbreviations we start with a short review of the leading-order results. For a detailed discussion we refer to Refs. [17, 19] and Ref. [24] where detector effects have been included in the simulation. As usual, the hadronic cross section is calculated from the partonic cross section through a convolution with the parton distribution functions (PDF),

$$
d \sigma_{p p \rightarrow t \bar{t} X}=\sum_{i, j} \int d x_{i} d x_{j} F_{i / p}\left(x_{i}, \mu_{f}\right) F_{j / p}\left(x_{j}, \mu_{f}\right) d \hat{\sigma}_{i j \rightarrow t \bar{t} X}\left(x_{i} P_{1}, x_{j} P_{2}, \mu_{f}, \mu_{r}\right),
$$

where the $F_{i / p}\left(x, \mu_{f}\right)$ denote the PDF, $\mu_{f}$ and $\mu_{r}$ are the factorization and renormalization scales, and $\hat{\sigma}_{i j \rightarrow t \bar{t} X}$ denote the partonic cross sections. The sum runs over all parton configurations contributing to top-quark pair production. Within QCD the dominant contributions are 
due to gluon fusion $(g g \rightarrow t \bar{t})$ and quark-antiquark annihilation $(q \bar{q} \rightarrow t \bar{t})$. The leading-order Feynman diagrams are shown in Fig. 1. The relative contributions of these two processes to
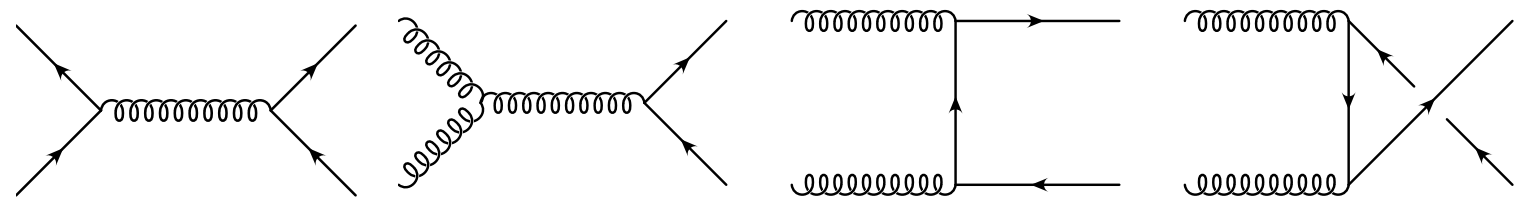

Figure 1: Dominant SM processes contributing to top-quark pair production.

(18) depend on the energy scale, with the contribution from gluon fusion dominating at low energies [52]. In Fig. 2 the additional contribution due to the $s$-channel exchange of a Higgs boson $\phi_{i}$ is shown. In principle, all quarks contribute in the fermion triangles. However in the scenarios considered here we have set $\tan \beta=0.7$. Thus the bottom Yukawa coupling is not enhanced (as would be the case in scenarios with $\tan \beta \gg 1$ ). That is why in these scenarios the bottom loop contribution is is more suppressed than in the SM with respect to the contribution from the top-quark loop. Contributions from quarks lighter than the $b$ quark are even smaller. Hence we can safely neglect all quark flavor contributions to the triangle which are lighter than the top quark. As we consider here an extended Higgs sector, we have to sum over the (neutral) Higgs bosons $\phi_{1}, \phi_{2}$, and $\phi_{3}$. We identify $\phi_{1}$ with the SM Higgs boson which con-
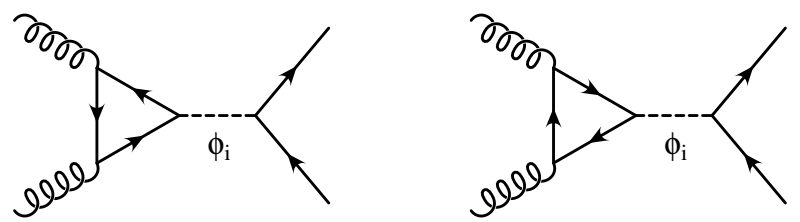

Figure 2: $s$-channel Higgs boson contribution to top-quark pair production.

tributes to the nonresonant background (17). Since we are mainly interested in the resonant production of the heavy Higgs bosons $\phi_{2}$ and $\phi_{3}$ we take at leading order only the contribution from the diagrams shown in Fig. 2 into account and neglect nonresonant Yukawa corrections due to $\phi_{2,3}$ exchange.

For $g g \rightarrow t \bar{t}$ the scattering amplitude may be written as

$$
\mathcal{A}=\mathcal{A}_{\mathrm{QCD}}^{(0)}+\sum_{i} \mathcal{A}_{\phi_{i}}^{(0)}
$$

and the partonic cross section can be calculated using

$$
d \sigma_{g g \rightarrow t \bar{t}}=\frac{1}{32 \pi} \frac{\beta_{t}}{S} \overline{|\mathcal{A}|^{2}} d \cos (\theta),
$$


where $s$ denotes the partonic center-of-mass energy squared, $\cos (\theta)$ is the cosine of the scattering angle in the parton center-of-mass system $(\mathrm{cms})$,

$$
\beta_{t}=\sqrt{1-\frac{4 m_{t}^{2}}{s}}
$$

is the velocity of the top quark in the partonic $\mathrm{cms}$, and $m_{t}$ denotes the top-quark mass renormalized in the on-shell scheme. We overline the squared matrix element in Eq. (20) to indicate that it is averaged/summed over the incoming/outgoing spin and color degrees of freedom. Squaring the amplitude for $g g \rightarrow t \bar{t}$ leads to

$$
\left|\mathcal{A}_{\mathrm{QCD}}^{(0)}\right|^{2}+2 \sum_{i} \operatorname{Re}\left(\mathcal{A}_{\mathrm{QCD}}^{(0)} \mathcal{A}_{\phi_{i}}^{(0)^{*}}\right)+2 \sum_{i>j} \operatorname{Re}\left(\mathcal{A}_{\phi_{i}}^{(0)} \mathcal{A}_{\phi_{j}}^{(0)^{*}}\right)+\sum_{i}\left|\mathcal{A}_{\phi_{i}}^{(0)}\right|^{2}
$$

For the scenarios $1-3$ discussed in Sec. 3 sizeable effects due to the extended Higgs sector can only be expected well above the threshold for top-quark pair production in the vicinity of the respective resonances $\phi_{2}, \phi_{3}$. (At these energies, the contribution of the SM-like Higgs boson $\phi_{1}$ is tiny and will, in fact, be incorporated in the following sections into the nonresonant background contributions.) The expression for the pure QCD matrix element squared $\left|\mathcal{A}_{\mathrm{QCD}}^{(0)}\right|^{2}$ in Eq. (22) can be found in Refs. [53, 54]. For the two heavy Higgs bosons the interference with the QCD amplitude reads

$$
2 \sum_{i=2,3} \overline{\operatorname{Re}\left(\mathcal{A}_{\mathrm{QCD}}^{(0)} \mathcal{A}_{\phi_{i}}^{(0)^{*}}\right)}=-\frac{16 \pi \alpha_{s}}{C_{A} C_{F}} \frac{m_{t}^{2}}{v} \frac{s}{1-\beta_{t}^{2} z^{2}} \sum_{i=2,3}\left[a_{i t} \beta_{t}^{2} \operatorname{Re}\left(P_{i}(s) \mathcal{F}_{i}^{S}\right)-2 b_{i t} \operatorname{Re}\left(P_{i}(s) \mathcal{F}_{i}^{P}\right)\right]
$$

with the propagator of the Higgs boson $\phi_{i}$ defined by

$$
P_{i}(s)=\frac{1}{s-m_{i}^{2}+i m_{i} \Gamma_{i}}
$$

and the $s$ dependent vertex factors given by

$$
\begin{aligned}
\mathcal{F}_{i}^{S} & =-\frac{\alpha_{s} a_{i t}}{8 \pi v} \tau[(1-\tau) f(\tau)-1] \\
\mathcal{F}_{i}^{P} & =\frac{\alpha_{s} b_{i t}}{16 \pi v} \tau f(\tau)
\end{aligned}
$$

where

$$
\tau=1-\beta_{t}^{2}=\frac{4 m_{t}^{2}}{s}
$$

The loop function $f$ is given by

$$
f(\tau)= \begin{cases}\frac{1}{4}\left[\ln \left(\frac{1+\sqrt{1-\tau}}{1-\sqrt{1-\tau}}\right)-i \pi\right]^{2} & \tau<1 \\ -\arcsin ^{2}\left(\sqrt{\frac{1}{\tau}}\right) & \tau \geq 1\end{cases}
$$


The $a_{i t}\left(b_{i t}\right)$ denote the reduced (pseudo-)scalar Yukawa couplings defined in Sec. 3, The $\Gamma_{i}$ are the constant widths of the $\phi_{i}$ as given in Sec. 3. $\alpha_{s} \equiv \alpha_{s}\left(\mu_{r}\right)$ is the coupling constant of the strong interaction evaluated at the renormalization scale $\mu_{r} . C_{A}$ and $C_{F}$ are the Casimir invariants of the $\mathrm{SU}(\mathrm{N})$ gauge group $(N=3$ for $\mathrm{QCD})$,

$$
C_{A}=N, \quad C_{F}=\frac{1}{2 N}\left(N^{2}-1\right),
$$

and $z$ is the cosine of the scattering angle of the top quark defined in the parton cms,

$$
z=\cos \left(\theta_{t}\right)
$$

The two remaining contributions in Eq. (22) read

$$
\begin{gathered}
\sum_{i=2,3} \overline{\left|\mathcal{A}_{\phi_{i}}^{(0)}\right|^{2}}=\frac{2}{C_{F}} \frac{m_{t}^{2}}{v^{2}} s^{3} \sum_{i=2,3}\left|P_{i}(s)\right|^{2}\left(\left|\mathcal{F}_{i}^{S}\right|^{2}+4\left|\mathcal{F}_{i}^{P}\right|^{2}\right)\left(a_{i t}^{2} \beta_{t}^{2}+b_{i t}^{2}\right), \\
\overline{2 \operatorname{Re}\left(\mathcal{A}_{\phi_{2}}^{(0)} \mathcal{A}_{\phi_{3}}^{(0)^{*}}\right)}=\frac{4}{C_{F}} \frac{m_{t}^{2}}{v^{2}} s^{3} \operatorname{Re}\left[P_{2}(s) P_{3}(s)^{*}\left(\mathcal{F}_{2}^{S} \mathcal{F}_{3}^{S^{*}}+4 \mathcal{F}_{2}^{P} \mathcal{F}_{3}^{P^{*}}\right)\right]\left(a_{2 t} a_{3 t} \beta_{t}^{2}+b_{2 t} b_{3 t}\right) .
\end{gathered}
$$

The interference term (32) is nonzero only in $C P$-violating scenarios, where $\phi_{2}, \phi_{3}$ have both scalar and pseudoscalar components. Although a detailed phenomenological discussion including higher order corrections will be presented in Sec. 5 it is useful to study the size of the different contributions (22) to the LO differential cross section (18). The cross section for top-quark pair production differential in the invariant mass $M_{t \bar{t}}$ of the top-quark pair is shown in Fig. 3 for scenario 3. As anticipated, sizeable effects due to the extended Higgs sector are only visible in the vicinity of the resonances. A distinctive peak-dip structure, which is due to the interference of the Higgs signal with the QCD background amplitude, is visible for the lighter of the two heavy Higgs resonances (which is $\phi_{2}$ in scenario 3). The heavier Higgs boson produces a similar peak-dip structure which is, however, less pronounced and hidden by the effect of the lighter resonance. As a consequence of the peak-dip structure the contribution of the extended Higgs sector to the total $t \bar{t}$ cross section is very small and below the currently attainable experimental accuracy. The effect can be enhanced by introducing a cut on $M_{t \bar{t}}$ and evaluating the cross sections in the regions $2 m_{t}<M_{t \bar{t}}<480 \mathrm{GeV}$ and $M_{t \bar{t}}>480$ $\mathrm{GeV}$. However, even in this case the effects are only at the level of a few percent. Qualitatively similar results are obtained for scenarios 1 and 2 .

Contrary to scenarios 1 and 2 the $\phi_{2}-\phi_{3}$ interference term is nonzero in scenario 3 . As shown in Fig. 3 this term is small compared to the other contributions; it is less than 0.4 per mill with respect to the QCD background in each $M_{t \bar{t}}$ bin. There are two reasons for this interference to be so small. First, in scenario 3 the Higgs boson mass separation $\Delta m=m_{3}-m_{2}>\Gamma_{2}+\Gamma_{3}$ leads to a suppression caused by the product of the two Higgs boson propagators in Eq. (32), while in the case $\Delta m=0$ there is no such suppression. This behavior is displayed in Fig. 4, where the red curve shows the relative contribution of the $\phi_{2}-\phi_{3}$ interference term to the LO hadronic $p p \rightarrow \phi_{2}, \phi_{3} \rightarrow t \bar{t}$ cross section without the QCD background as a function of the mass separation $\Delta m=m_{3}-m_{2}$. Second, even in the case $\Delta m=0$ the $\phi_{2}-\phi_{3}$ interference term is still small (about $4 \%$ compared to the Higgs-only cross section). This is the result of our choice 


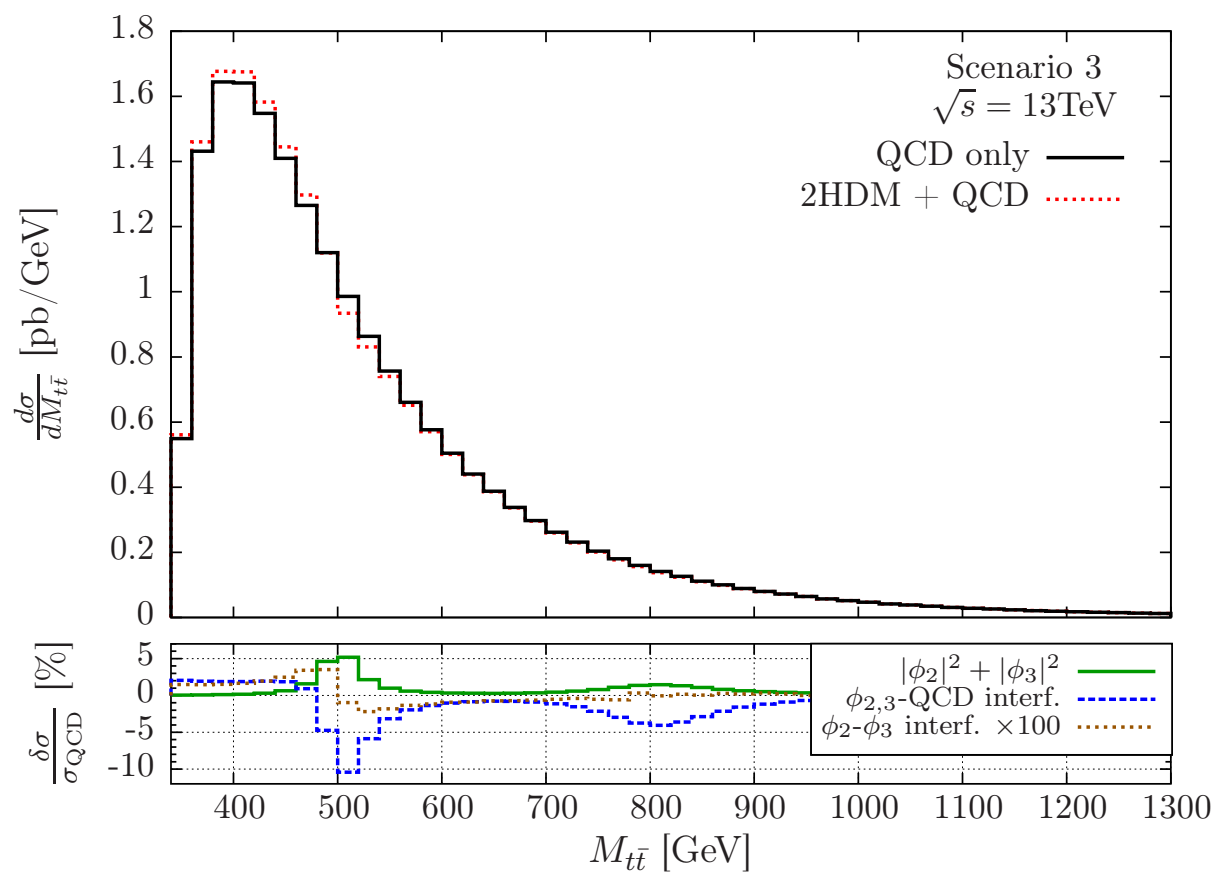

Figure 3: Impact of different contributions to the hadronic differential cross section $d \sigma / d M_{t \bar{t}}$ at leading order. Upper pane: The solid black curve shows the QCD background and the dotted red curve displays the sum of QCD background, 2HDM contribution and the interference term. Lower pane: Relative contributions with respect to the QCD background. The solid green curve shows the sum of the contributions from $\phi_{2}$ and $\phi_{3}$, the dashed blue curve depicts the interference of the $\phi_{2}, \phi_{3}$ production amplitudes with the QCD background, and the dotted brown curve the $\phi_{2}-\phi_{3}$ interference (multiplied with a factor 100 for better visibility). 
of parameters for scenario 3, which leads to Yukawa couplings with opposite signs. This is in turn responsible for cancellations in Eq. (32) and renders the interference term small. The blue curve in Fig. 4 shows in addition the behavior of this interference term as a function of the Higgs mass separation using an additional cut, namely $m_{2}-150 \mathrm{GeV} \leq M_{t \bar{t}} \leq m_{2}$. This cut enhances the $\phi_{2}-\phi_{3}$ interference because it prevents the partial cancellation due to the peakdip structure which is also present in this interference term. We apply similar cuts in the NLO analysis presented below. Even with this cut the interference contribution Eq. (32) is small compared to the total Higgs boson contributions to the $t \bar{t}$ cross section and can be safely neglected in phenomenological applications.

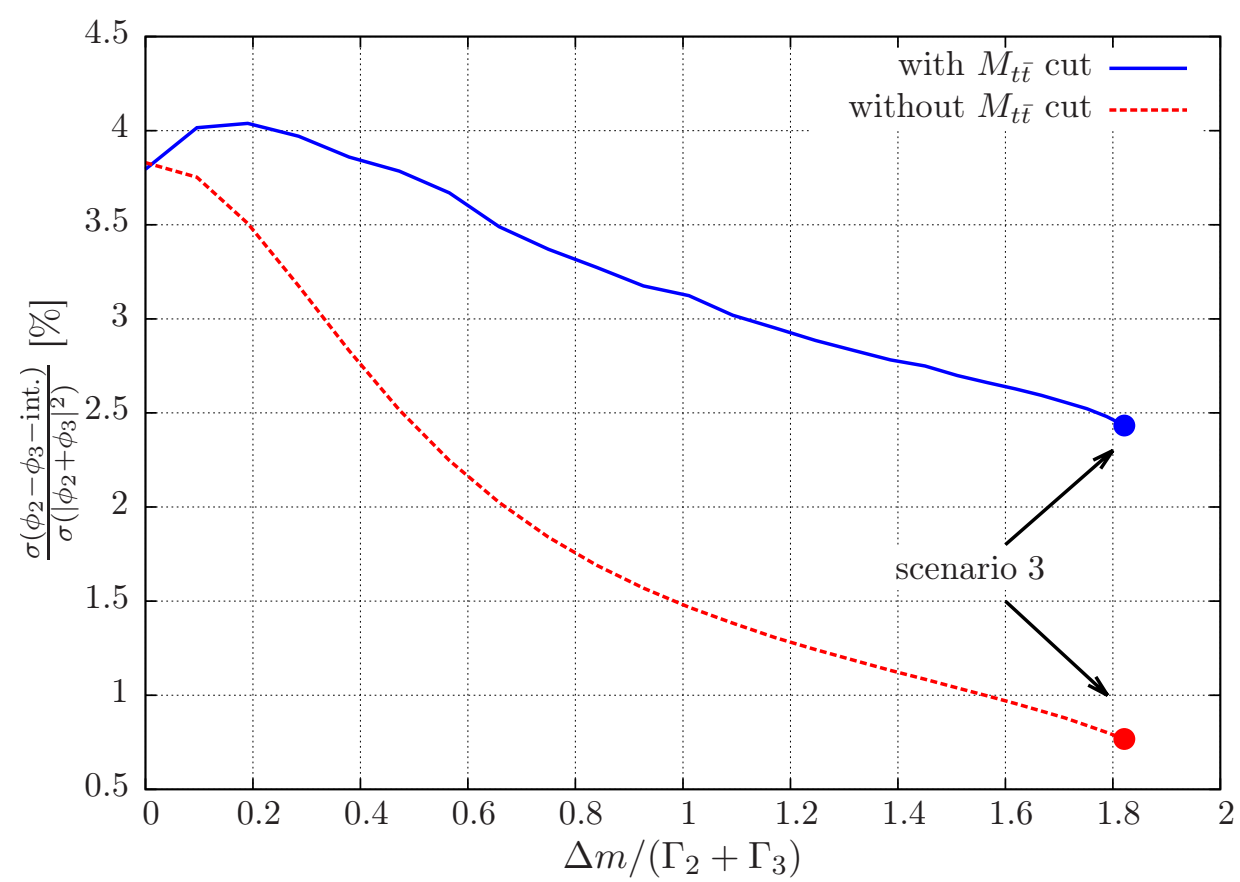

Figure 4: Relative contribution of the $\phi_{2}-\phi_{3}$-interference term 32 with respect to the total hadronic $p p \rightarrow \phi_{2}, \phi_{3} \rightarrow t \bar{t}$ cross section without QCD background as a function of the mass separation $\Delta m=m_{3}-m_{2}$ normalized to $\Gamma_{2}+\Gamma_{3}$. The parameters used for this plot correspond to those of scenario 3 except for the masses and widths of $\phi_{2}$ and $\phi_{3}$ which have been varied accordingly. The end of each curve corresponds exactly to scenario 3 with $m_{2}=500 \mathrm{GeV}, m_{3}=800 \mathrm{GeV}$ and corresponding widths as given in Table 6 .

In order to find or put limits on heavy Higgs boson production and decay to $t \bar{t}$ the experimental analysis must focus on the resonance region. Since the mass(es) of the heavy resonance(s) is (are) not known beforehand, an analysis with a sliding $M_{t \bar{t}}$ window combined with a sideband analysis should be applied. From the theoretical point of view NLO corrections are required to provide reliable predictions in the vicinity of the resonances. The evaluation of the NLO corrections will be discussed in the next subsection. 
Besides we remark that, depending on the mass, couplings and width of the heavy Higgs resonance, a pure dip rather than a peak dip may show up in the $M_{t \bar{t}}$ spectrum, or even a complete washout of the resonance signal can occur. For a recent analysis, see Ref. [25]. For the parameter scenarios described in Sec. 3 the lighter of the two heavy Higgs resonances produces always a peak-dip structure in the $M_{t \bar{t}}$ distribution; see Sec. 5

\subsection{Next-to-leading order QCD corrections}

For the calculation of the NLO QCD corrections to the process in Eq. (16) we apply two approximations. First, as far as the Higgs coupling to the gluons is concerned, we work in the heavy top-quark limit. Second, we take advantage of the fact that the theoretical predictions are only required in the vicinity of the resonances because the experimental analysis will focus on this region. This allows us to work in the pole approximation, keeping only contributions that are resonant in the intermediate Higgs boson(s). This is consistent with our approach to work at leading order in the Yukawa couplings. The heavy Higgs resonances are described by a Breit-Wigner propagator with constant width. In our approach this amounts to using the so-called complex mass scheme [55-57], as outlined in Appendix A

In order to fix our conventions we briefly review the approximations in the following.

Effective theory approach: In the heavy top-quark limit the triangles shown in Fig. 2 are shrunk to an effective vertex $\sqrt{3}^{3}$ Relying on Lorentz covariance and gauge invariance, the effective vertex can be described within an effective field theory approach with the relevant contribution to the Lagrangian given by

$$
\mathcal{L}_{\mathrm{eff}}=\sum_{j=2}^{3}\left[f_{S j} G_{\mu \nu}^{a} G_{a}^{\mu \nu}+f_{P j} \epsilon_{\mu \nu \alpha \beta} G_{a}^{\mu v} G_{a}^{\alpha \beta}\right] \phi_{j},
$$

where $f_{S j}$ and $f_{P j}$ are the Wilson coefficients for the coupling of the $C P$-even and $C P$-odd component of the Higgs boson $\phi_{j}$ to gluons. The effective Lagrangian leads to the following Feynman rules $\left(g_{s}=\sqrt{4 \pi \alpha_{s}}\right)$

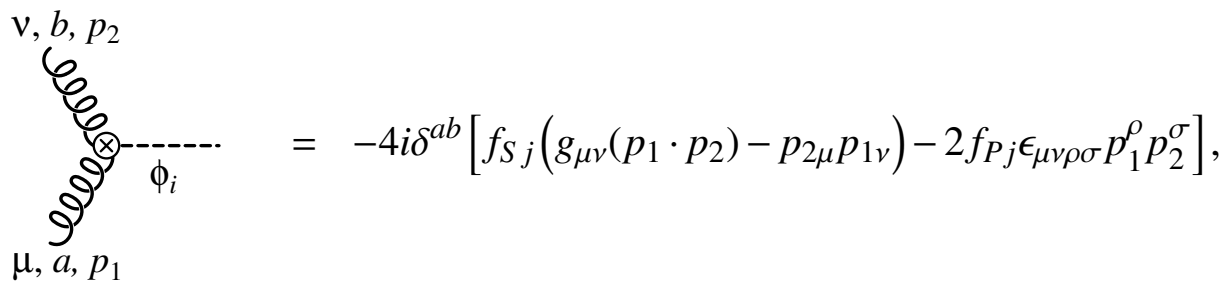

\footnotetext{
${ }^{3}$ The virtual NLO QCD corrections for scalar and pseudoscalar Higgs-boson production in gluon fusion were calculated for arbitrary quark masses in Ref. [58].
} 


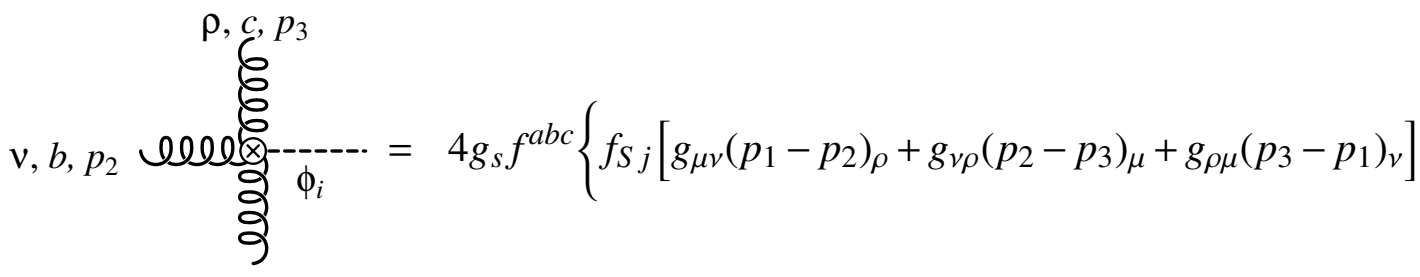

$$
\begin{aligned}
& \mu, a, p_{1} \\
& \left.-2 f_{P j}\left(p_{1}+p_{2}+p_{3}\right)^{\alpha} \epsilon_{\alpha \mu \nu \rho}\right\} .
\end{aligned}
$$

The momenta are taken to be incoming and we use the convention $\epsilon_{0123}=+1$. The Lagrangian $\mathcal{L}_{\text {eff }}$ contains also an effective $\operatorname{gggg} \phi_{j}$ coupling, which is however not needed at the order of perturbation theory in which we are working. The effective couplings $f_{S j}$ and $f_{P j}$ can be further expanded in powers of $\alpha_{s}$ :

$$
f_{S j}=\frac{\alpha_{S}}{\pi}\left(f_{S j}^{(0)}+\frac{\alpha_{S}}{\pi} f_{S j}^{(1)}+\ldots\right), \quad f_{P j}=\frac{\alpha_{S}}{\pi}\left(f_{P j}^{(0)}+\frac{\alpha_{S}}{\pi} f_{P j}^{(1)}+\ldots\right) .
$$

Comparing the results obtained in the full theory as presented in the previous subsection with the results in the effective theory approach, it is straightforward to obtain the well-known result:

$$
\begin{aligned}
\frac{\alpha_{s}}{\pi} f_{S j}^{(0)} & =\lim _{m_{t} \rightarrow \infty} \mathcal{F}_{j}^{S}=\frac{\alpha_{S}}{\pi} \frac{a_{j t}}{12 v}, \\
\frac{\alpha_{s}}{\pi} f_{P j}^{(0)} & =\lim _{m_{t} \rightarrow \infty} \mathcal{F}_{j}^{P}=-\frac{\alpha_{S}}{\pi} \frac{b_{j t}}{16 v} .
\end{aligned}
$$

Using the expressions from Ref. [59-62] adapted to our conventions we get for $f_{S j}^{(1)}$ and $f_{P j}^{(1)}$ :

$$
\begin{aligned}
f_{S j}^{(1)} & =\frac{(4 \pi)^{\epsilon}}{\Gamma(1-\epsilon)} \frac{a_{j t}}{12 v}\left(\frac{11}{4}-\frac{\beta_{0}}{\epsilon}\right)=\frac{(4 \pi)^{\epsilon}}{\Gamma(1-\epsilon)} f_{S j}^{(0)}\left(\frac{11}{4}-\frac{\beta_{0}}{\epsilon}\right), \\
f_{P j}^{(1)} & =\frac{(4 \pi)^{\epsilon}}{\Gamma(1-\epsilon)} \frac{b_{j t}}{16 v} \frac{\beta_{0}}{\epsilon}=-\frac{(4 \pi)^{\epsilon}}{\Gamma(1-\epsilon)} f_{P j}^{(0)} \frac{\beta_{0}}{\epsilon}
\end{aligned}
$$

where $\epsilon=(4-d) / 2$ is the dimensional regulator working in $d$ dimensions, and

$$
\beta_{0}=\frac{1}{2}\left(\frac{11}{6} C_{A}-\frac{2}{3} T_{R} N_{F}\right)
$$

denotes the leading coefficient of the QCD $\beta$-function with $N_{F}$ being the number of massless quark flavors and $T_{R}=1 / 2$. One may question the validity of the heavy top-quark limit given that we apply the effective theory approach above the threshold of top-quark pair production. Since the effective theory approach has been shown to provide also reliable estimates for energies well above its naive domain of validity-if the full leading-order result is kept and an appropriate reweighting is performed [29]-we believe that reliable estimates of the NLO corrections can be obtained within this approximation. We outline our reweighting method adapted from Ref. [29] at the end of this section. 
Pole contribution and soft gluon approximation: As sizeable effects of the extended Higgs sector can be expected only in the heavy Higgs boson resonance region it is sufficient to restrict the theoretical predictions to $M_{t \bar{t}}$ intervals around the Higgs boson masses. This can be achieved by the pole approximation where only the contributions enhanced by a resonant propagator are kept. For Higgs propagators appearing within loop diagrams the soft gluon

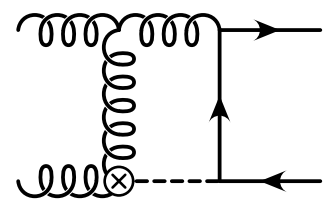

Figure 5: Diagram to be calculated in the soft gluon approximation.

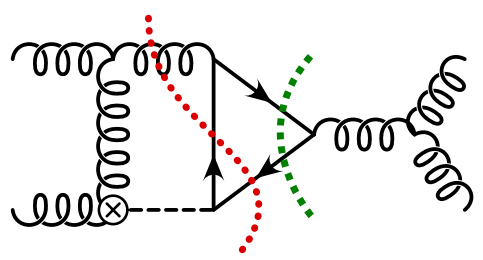

Figure 6: Virtual (green dashed) and real (red dotted) contributions which cancel in the soft gluon approximation.

approximation can be used to extract the leading pole contribution. Taking as an example the diagram shown in Fig. 5 it is clear that a resonant contribution at $M_{t \bar{t}} \approx m_{i}$ is only possible if the additional gluon in the loop is soft. This is the essence of the soft gluon approximation in which the integrand of the loop integral is approximated by the contribution from soft gluons. Note that the loop integration is not restricted within this approximation. For more details we refer to Refs. [63-66]. Evidently, the same approximation has to be applied also to the real corrections. In fact, the virtual corrections alone are infrared divergent because the soft gluon is coupled to external lines. Only the sum of the virtual and real corrections yields a finite result. In the soft gluon limit the amplitudes simplify significantly and it turns out that the appropriate real corrections completely cancel the corresponding virtual contributions once sufficiently inclusive observables are studied. An example of this cancellation is illustrated in Fig. 6. This was checked for the various contributions on a case by case study. More details about the example shown in Fig. 6 are given in Appendix B. The corrections of the type displayed in Figs. 5-6 are also called nonfactorizable corrections because the gluon radiation connects Higgs boson production and decay to $t \bar{t}$. We also stress that gauge invariance is only guaranteed if the soft gluon approximation is consistently applied.

After these introductory remarks we now briefly summarize the various terms that contribute, within the aforementioned approximations, to resonant $\phi_{j}$ production and interference with the nonresonant $t \bar{t}$ amplitudes at NLO QCD, i.e., at order $\alpha_{s}^{3}$. In Fig. 7 the factorizing QCD 

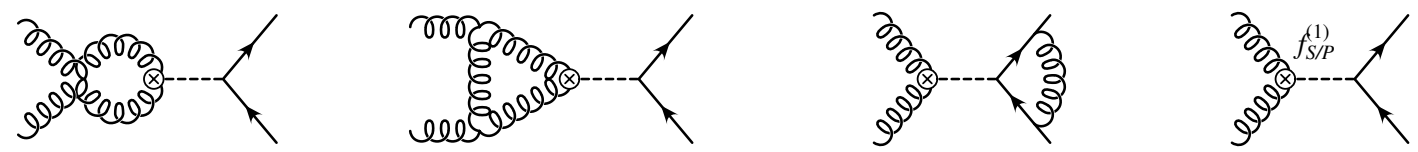

Figure 7: QCD corrections to top-quark pair production via resonant Higgs exchange.

corrections for top-quark pair production through Higgs exchange are shown and we do not display diagrams that vanish in dimensional regularization. Denoting the amplitude for this contribution with $\mathcal{A}_{\phi_{i}}^{(1)}$ and the QCD corrections to nonresonant top-quark pair production (without any Higgs contribution) with $\mathcal{A}_{\mathrm{QCD}}^{(1)}$, the virtual corrections to the squared matrix element read

$$
\sum_{i, j} 2 \operatorname{Re}\left(\mathcal{A}_{\phi_{i}}^{(1)} \mathcal{A}_{\phi_{j}}^{(0)^{*}}\right)+\sum_{i} 2 \operatorname{Re}\left(\mathcal{A}_{\phi_{i}}^{(1)} \mathcal{A}_{\mathrm{QCD}}^{(0)^{*}}\right)+\sum_{i} 2 \operatorname{Re}\left(\mathcal{A}_{\mathrm{QCD}}^{(1)} \mathcal{A}_{\phi_{i}}^{(0)^{*}}\right)
$$

We refrain from discussing the NLO QCD corrections to $t \bar{t}$ production here, because they have been known for a long time [53, 54, 67-70]. (In [69, 70] the full spin dependence of the top quarks is kept.) After renormalization the virtual corrections are ultraviolet (UV) finite. However, they still contain infrared (IR) singularities which are canceled only after the inclusion of the real corrections. Sample diagrams for $g g \rightarrow \phi_{i} \rightarrow t \bar{t} g$ are shown in Fig. 8. The square of this amplitude cancels the IR singularities in $2 \operatorname{Re}\left(\mathcal{A}_{\phi_{i}}^{(1)} \mathcal{A}_{\phi_{j}}^{(0)^{*}}\right)$. Because of the
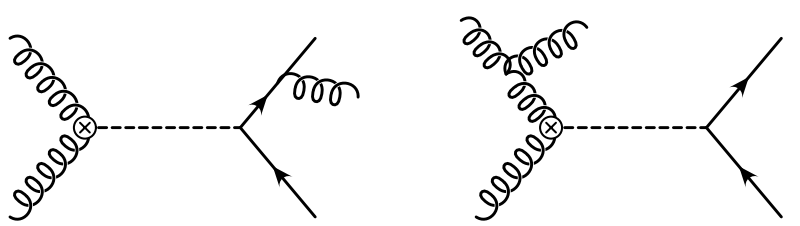

Figure 8: Sample diagrams for initial and final state real corrections to the process $g g \rightarrow \phi_{2,3} \rightarrow t \bar{t}$.

color structure there is no interference between initial and final state radiation in this case. The amplitude for $g g \rightarrow \phi_{i} \rightarrow t \bar{t} g$ interferes also with the QCD amplitude $g g \rightarrow t \bar{t} g$. This contribution cancels the IR singularities in $\sum_{i} 2 \operatorname{Re}\left(\mathcal{A}_{\phi_{i}}^{(1)} \mathcal{A}_{\mathrm{QCD}}^{(0)^{*}}\right)$ and $\sum_{i} 2 \operatorname{Re}\left(\mathcal{A}_{\mathrm{QCD}}^{(1)} \mathcal{A}_{\phi_{i}}^{(0)^{*}}\right)$. We stress that the contribution to be combined with $\sum_{i} 2 \operatorname{Re}\left(\mathcal{A}_{\phi_{i}}^{(1)} \mathcal{A}_{\mathrm{QCD}}^{(0)^{*}}\right)$ is evaluated using the soft gluon approximation.

At order $\alpha_{s}^{3}$ heavy Higgs bosons can also be produced by $q \bar{q}$ annihilation with an amplitude that is not suppressed by light quark Yukawa couplings. The respective Feynman diagram for $q \bar{q} \rightarrow t \bar{t} g$ is shown in Fig. 9 . Besides the square of this amplitude also the interference with the QCD amplitude for $q \bar{q} \rightarrow t \bar{t} g$ contributes. Both contributions are free of IR singularities.

In addition, resonant heavy Higgs boson production and decay to $t \bar{t}$ occurs also by (anti)quark gluon fusion, $q g \rightarrow t \bar{t} q$ and $g \bar{q} \rightarrow t \bar{t} \bar{q}$. The corresponding amplitudes are obtained from the 


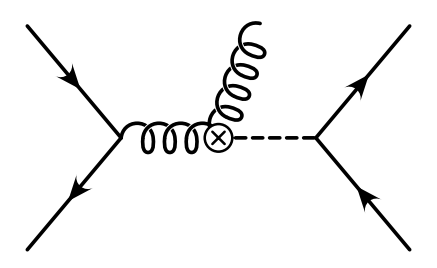

Figure 9: Resonant heavy Higgs boson production in $q \bar{q} \rightarrow t \bar{t} g$.

amplitude depicted in Fig. 9 by crossing. The square of the amplitude with the intermediate Higgs bosons and the interference with the respective QCD amplitude lead to double and single resonant terms. Contrary to the $q \bar{q}$ case both contributions suffer from initial state singularities.

Some technical aspects: The calculation of the NLO corrections described above is conceptually straightforward. Since the full results are rather lengthy we briefly outline the calculation and show explicit results for some illustrative examples only. As far as the virtual corrections are concerned we employ the Passarino-Veltman reduction [71] to reduce the oneloop tensor integrals to scalar integrals. Including the contribution from UV renormalization we obtain for example for the interference of the diagrams shown in Fig. 7 and the leadingorder Higgs boson exchange diagrams

$$
\begin{aligned}
\sum_{i, j} \overline{2 \operatorname{Re}\left(\mathcal{A}_{\phi_{i}}^{(1)} \mathcal{A}_{\phi_{j}}^{(0)^{*}}\right)} & =\frac{\alpha_{s}}{\pi}\left\{-\frac{(4 \pi)^{\epsilon}}{\Gamma(1-\epsilon)}\left\{\operatorname{Re}\left[\left(\frac{\mu^{2}}{-s-i 0}\right)^{\epsilon}\right] \frac{C_{A}}{\epsilon^{2}}+\frac{2 \beta_{0}}{\epsilon}\right\} \mathcal{B}\right. \\
& +\left(\frac{4 \pi \mu^{2}}{m_{t}^{2}}\right)^{\epsilon} \Gamma(1+\epsilon) C_{F} \frac{1}{\epsilon} \operatorname{Re}\left(a_{-1}\right) \mathcal{B} \\
& +\left.\frac{11}{2} \mathcal{B}\right|_{f_{P}=0}+\left.2 C_{A} \mathcal{B}\right|_{f_{S}=0} \\
& \left.+C_{F}\left[\left.\operatorname{Re}\left(a_{0}\right) \mathcal{B}\right|_{b_{t}=0}+\left.\operatorname{Re}\left(\bar{a}_{0}\right) \mathcal{B}\right|_{a_{t}=0}\right]+O(\epsilon)\right\},
\end{aligned}
$$

where $\mathcal{B}$ denotes the contribution from

$$
\sum_{i=2,3} \overline{\left|\mathcal{A}_{\phi_{i}}^{(0)}\right|^{2}}+2 \overline{\operatorname{Re}\left(\mathcal{A}_{\phi_{2}}^{(0)} \mathcal{A}_{\phi_{3}}^{(0)^{*}}\right)}
$$

evaluated in the heavy top-quark limit:

$$
\begin{aligned}
\mathcal{B} & =\frac{2}{C_{F}}\left(\frac{\alpha_{s}}{\pi}\right)^{2} \frac{m_{t}^{2}}{v^{2}} s^{3}\left\{\sum_{i=2,3}\left|P_{i}(s)\right|^{2}\left(\left|f_{S i}^{(0)}\right|^{2}+4\left|f_{P i}^{(0)}\right|^{2}\right)\left(a_{i t}^{2} \beta_{t}^{2}+b_{i t}^{2}\right)\right. \\
& \left.+2 \operatorname{Re}\left[P_{2}(s) P_{3}(s)^{*}\left(f_{S 2}^{(0)} f_{S 3}^{(0)^{*}}+4 f_{P 2}^{(0)} f_{P 3}^{(0)^{*}}\right)\right]\left(a_{2 t} a_{3 t} \beta_{t}^{2}+b_{2 t} b_{3 t}\right)\right\} .
\end{aligned}
$$


The coefficients $a_{-1}, a_{0}$ and $\bar{a}_{0}$ are related to the one-loop $\phi_{j} \rightarrow t \bar{t}$ form factor and can be taken for example from Ref. [72]. The poles in the dimensional regulator $\epsilon$ are due to IR singularities. Note that we keep the polarization of the incoming gluons in four dimensions. As a consequence $\mathcal{B}$ does not depend on $\epsilon$. The IR singularities are handled with the CataniSeymour dipole subtraction [73,74]. In this formalism local counterterms are added to the real corrections such that on the one hand the soft and collinear singularities are canceled pointwise in phase space and, on the other hand, the subtraction is simple enough to be integrated analytically over the $d$-dimensional phase space of the unobserved parton. The cross section reads schematically [73]:

$$
\begin{aligned}
\sigma^{\mathrm{NLO}} & =\sigma^{\mathrm{NLO}\{m+1\}}(p)+\sigma^{\mathrm{NLO}\{m\}}(p)+\int_{0}^{1} d x d \hat{\sigma}^{\mathrm{NLO}\{m\}}(x ; x p) \\
& =\int_{m+1}\left[\left(\sigma^{\mathrm{R}}(p)\right)_{\epsilon=0}-\left(\sum_{\text {dipoles }} d \sigma^{\mathrm{B}}(p) \otimes\left(d V_{\text {dipole }}+d V_{\text {dipole }}^{\prime}\right)\right)_{\epsilon=0}\right] \\
& +\int_{m}\left[d \sigma^{\mathrm{V}}(p)+d \sigma^{\mathrm{B}}(p) \otimes \mathbf{I}\right]_{\epsilon=0}+\int_{0}^{1} d x \int_{m}\left[d \sigma^{\mathrm{B}}(x p) \otimes(\mathbf{K}+\mathbf{P})(x)\right] .
\end{aligned}
$$

Here $\sigma^{\mathrm{B}}, \sigma^{\mathrm{V}}$, and $\sigma^{\mathrm{R}}$ denote the Born cross section and the contributions from the virtual and real corrections, respectively. Since the real corrections combined with the subtraction terms are finite, they can be evaluated numerically in four dimensions. The singularities are thus made manifest in the integrated subtraction terms which are added back [last line in Eq. (45)]. The subtraction term to be combined with the real corrections is constructed as a sum of individual dipoles:

$$
\sum_{\text {dipoles }} d \sigma^{\mathrm{B}}(p) \otimes\left(d V_{\text {dipole }}+d V_{\text {dipole }}^{\prime}\right) .
$$

The analytically integrated dipoles are collected in the operators $\mathbf{I}, \mathbf{K}$, and $\mathbf{P}$ which may introduce correlations in spin and color. Combining for the case at hand the virtual corrections with the contribution from the I operator an IR finite result is obtained:

$$
\begin{aligned}
d \sigma_{V}+d \sigma_{\mathbf{I}} & =\frac{1}{32 \pi} \frac{\alpha_{s}}{\pi} \frac{\beta_{t}}{s}\left\{\left[2 \beta_{0}+\frac{67}{18} C_{A}-\frac{10}{9} N_{F} T_{R}\right.\right. \\
& +C_{F}\left(3-\frac{\beta_{t}^{2}+6 \beta_{t}+1}{12 \beta_{t}} \pi^{2}-\frac{1+\beta_{t}^{2}}{4 \beta_{t}} \ln ^{2} y-\frac{1+\beta_{t}^{2}}{2 \beta_{t}} \ln \left(\frac{1+\beta_{t}^{2}}{2}\right) \ln (y)\right. \\
& \left.\left.+\left(\frac{1+\beta_{t}^{2}}{2 \beta_{t}} \ln (y)+\frac{3}{2}\right) \ln \left(\frac{1-\beta_{t}^{2}}{2\left(1+\beta_{t}^{2}\right)}\right)+\mathcal{V}_{q}^{(\mathrm{NS})}\right)+2 \beta_{0} \ln \left(\frac{\mu^{2}}{s}\right)\right] \mathcal{B} \\
& +\left.2 C_{A} \mathcal{B}\right|_{f_{S}=0}+\left.\frac{11}{2} \mathcal{B}\right|_{f_{S}=0} \\
& \left.+C_{F}\left[\left.\operatorname{Re}\left(a_{0}\right) \mathcal{B}\right|_{b_{t}=0}+\left.\operatorname{Re}\left(\bar{a}_{0}\right) \mathcal{B}\right|_{a_{t}=0}\right]\right\} d \cos (\theta)
\end{aligned}
$$


with

$$
\begin{aligned}
\mathcal{V}_{q}^{(\mathrm{NS})} & =\frac{3}{2} \ln \left(\frac{1}{2}\left(1+\beta_{t}^{2}\right)\right) \\
& +\frac{1+\beta_{t}^{2}}{2 \beta_{t}}\left(2 \ln (y) \ln \left(\frac{2\left(1+\beta_{t}^{2}\right)}{\left(1+\beta_{t}\right)^{2}}\right)+2 \operatorname{Li}_{2}\left(y^{2}\right)-2 \operatorname{Li}_{2}\left(\frac{2 \beta_{t}}{1+\beta_{t}}\right)-\frac{\pi^{2}}{6}\right) \\
& +\ln \left(1-\frac{1}{2} \sqrt{1-\beta_{t}^{2}}\right)-2 \ln \left(1-\sqrt{1-\beta_{t}^{2}}\right)-\frac{1-\beta_{t}^{2}}{1+\beta_{t}^{2}} \ln \left(\frac{\sqrt{1-\beta_{t}^{2}}}{2-\sqrt{1-\beta_{t}^{2}}}\right) \\
& -\frac{\sqrt{1-\beta_{t}^{2}}}{2-\sqrt{1-\beta_{t}^{2}}}+2 \frac{\left(1-\sqrt{1-\beta_{t}^{2}}-\beta_{t}^{2}\right)}{1+\beta_{t}^{2}}+\frac{\pi^{2}}{2}
\end{aligned}
$$

and

$$
y=\frac{1-\beta_{t}}{1+\beta_{t}} .
$$

The sum of the contributions from the integrated dipoles responsible for initial state singularities and the QCD factorization reads

$$
\begin{aligned}
d \sigma_{\mathbf{K P}} & =\int_{0}^{1} d x \int_{m}\left[d \sigma^{\mathrm{B}}(x p) \otimes(\mathbf{K}+\mathbf{P})(x)\right] \\
& =\frac{\alpha_{s}}{\pi} \int_{0}^{1} d x \int d \Pi_{2}\left\{\bar{K}^{g g}(x)-K_{\mathrm{F} . S .}^{g g}(x)+\widetilde{K}^{g g}(x)-P^{g g}(x) \ln \left(\frac{\mu_{F}^{2}}{x s}\right)\right\} \mathcal{B}(x s) .
\end{aligned}
$$

In the evaluation of $\mathcal{B}(x s)$ the partonic center-of-mass energy squared is set to $x s$. Since we factorize the initial state singularities in the $\overline{\mathrm{MS}}$ scheme the contribution $K_{\mathrm{F} . \mathrm{S}}^{g g}(x)$ encoding the scheme dependence for schemes different from the $\overline{\mathrm{MS}}$ scheme is zero. For the definition of the remaining contributions we refer to Ref. [73].

For the real corrections very short expressions can be derived for the squared Higgs boson contribution. In the reaction

$$
g\left(p_{1}\right)+g\left(p_{2}\right) \rightarrow t\left(k_{1}\right)+\bar{t}\left(k_{2}\right)+g(q)
$$

the $t \bar{t}$ pair is produced in a color singlet state. Therefore, the squared matrix element is given by an incoherent sum of two terms associated with gluon radiation from the initial and final state. We find

$$
\begin{aligned}
\overline{\left|\mathcal{A}\left(g g \stackrel{\phi_{2}, \phi_{3}}{\longrightarrow} t \bar{t} g\right)\right|_{\mathrm{ISR}}^{2}} & =\frac{16 \alpha_{s}^{3} C_{A}}{\pi C_{F}} \frac{m_{t}^{2}}{v^{2}} \frac{(s+t+u)^{4}+s^{4}+t^{4}+u^{4}}{s t u} \\
& \times\left\{\sum_{j=2,3}\left(\left(f_{S j}^{(0)}\right)^{2}+4\left(f_{P j}^{(0)}\right)^{2}\right)\left(-a_{j t}^{2} t_{12}+b_{j t}^{2} s_{12}\right)\left|P_{j}\left(s_{12}\right)\right|^{2}\right. \\
& \left.+2\left(f_{S 2}^{(0)} f_{S 3}^{(0)}+4 f_{P 2}^{(0)} f_{P 3}^{(0)}\right)\left(-a_{2 t} a_{3 t} t_{12}+b_{2 t} b_{3 t} s_{12}\right) \operatorname{Re}\left[P_{23}\left(s_{12}\right)\right]\right\}
\end{aligned}
$$


with

$$
s=\left(p_{1}+p_{2}\right)^{2}, \quad t=\left(p_{1}-q\right)^{2}, \quad u=\left(p_{2}-q\right)^{2}, \quad s_{12}=\left(k_{1}+k_{2}\right)^{2}, \quad t_{12}=\left(k_{1}-k_{2}\right)^{2},
$$

and

$$
P_{23}(s)=P_{2}(s) P_{3}(s)^{*} .
$$

The contribution from final state gluon radiation reads

$$
\begin{aligned}
\overline{\left|\mathcal{A}\left(g g \stackrel{\phi_{2}, \phi_{3}}{\longrightarrow} t \bar{t} g\right)\right|_{\mathrm{FSR}}^{2}} & =\frac{16 \alpha_{s}^{3} s^{2}}{\pi s_{13}^{\prime 2} s_{23}^{\prime 2}} \frac{m_{t}^{2}}{v^{2}}\left\{\sum_{j=2,3}\left[\left(f_{S j}^{(0)}\right)^{2}+4\left(f_{P j}^{(0)}\right)^{2}\right]\left[a_{j t}^{2} K_{a}+b_{j t}^{2} K_{b}\right]\left|P_{j}(s)\right|^{2}\right. \\
& \left.+2\left[f_{S 2}^{(0)} f_{S 3}^{(0)}+4 f_{P 2}^{(0)} f_{P 3}^{(0)}\right]\left[a_{2 t} a_{3 t} K_{a}+b_{2 t} b_{3 t} K_{b}\right] \operatorname{Re}\left[P_{23}(s)\right]\right\}
\end{aligned}
$$

with

$$
\begin{aligned}
& K_{a}=8 m_{t}^{4}\left(s-s_{12}\right)^{2}-2 m_{t}^{2}\left(s\left(s_{13}^{\prime}+s_{23}^{\prime}\right)^{2}+4 s_{12} s_{13}^{\prime} s_{23}^{\prime}\right)+s_{13}^{\prime} s_{23}^{\prime}\left(s^{2}+s_{12}^{2}\right), \\
& K_{b}=s_{13}^{\prime} s_{23}^{\prime}\left(s^{2}+s_{12}^{2}\right)-2 m_{t}^{2} s\left(s-s_{12}\right)^{2},
\end{aligned}
$$

and

$$
s_{13}^{\prime}=2 k_{1} \cdot q, \quad s_{23}^{\prime}=2 k_{2} \cdot q \text {. }
$$

For the quark initiated processes

$$
q\left(p_{1}\right)+\bar{q}\left(p_{2}\right) \rightarrow t\left(k_{1}\right)+\bar{t}\left(k_{2}\right)+g(q)
$$

and

$$
q\left(p_{1}\right)+g\left(p_{2}\right) \rightarrow t\left(k_{1}\right)+\bar{t}\left(k_{2}\right)+q(q)
$$

the squared amplitudes without the QCD interference are given by

$$
\begin{aligned}
\overline{\left|\mathcal{A}\left(q g \stackrel{\phi_{2}, \phi_{3}}{\longrightarrow} t \bar{t} q\right)\right|^{2}} & =\frac{16 \alpha_{s}^{3}}{\pi} \frac{m_{t}^{2}}{v^{2}} \frac{s^{2}+u^{2}}{-t}\left\{\sum_{j=2,3}\left[\left(f_{S j}^{(0)}\right)^{2}+4\left(f_{P j}^{(0)}\right)^{2}\right]\left(-a_{j t}^{2} t_{12}+b_{j t}^{2} s_{12}\right)\left|P_{j}\left(s_{12}\right)\right|^{2}\right. \\
& \left.+2\left[f_{S 2}^{(0)} f_{S 3}^{(0)}+4 f_{P 2}^{(0)} f_{P 3}^{(0)}\right]\left(-a_{2 t} a_{3 t} t_{12}+b_{2 t} b_{3 t} s_{12}\right) \operatorname{Re}\left[P_{23}\left(s_{12}\right)\right]\right\}
\end{aligned}
$$

and

$$
\begin{aligned}
\overline{\left|\mathcal{A}\left(q \bar{q} \stackrel{\phi_{2}, \phi_{3}}{\longrightarrow} t \bar{t} g\right)\right|^{2}} & =\frac{32 \alpha_{s}^{3} C_{F}}{\pi} \frac{m_{t}^{2}}{v^{2}} \frac{t^{2}+u^{2}}{s}\left\{\sum_{j=2,3}\left[\left(f_{S j}^{(0)}\right)^{2}+4\left(f_{P j}^{(0)}\right)^{2}\right]\left(-a_{j t}^{2} t_{12}+b_{j t}^{2} s_{12}\right)\left|P_{j}\left(s_{12}\right)\right|^{2}\right. \\
& \left.+2\left[f_{S 2}^{(0)} f_{S 3}^{(0)}+4 f_{P 2}^{(0)} f_{P 3}^{(0)}\right]\left(-a_{2 t} a_{3 t} t_{12}+b_{2 t} b_{3 t} s_{12}\right) \operatorname{Re}\left[P_{23}\left(s_{12}\right)\right]\right\} .
\end{aligned}
$$

The interferences of the signal amplitudes with the nonresonant QCD amplitudes produce rather lengthy expressions and we do not reproduce them here. As far as the NLO QCD and 
weak corrections to $t \bar{t}$ without contributions from $\phi_{2}$ and $\phi_{3}$ are concerned, we use the results of our previous work [69,70,75]. As mentioned above, the (small) contribution from the SM-like Higgs boson $\phi_{1}(125 \mathrm{GeV})$ is part of the order $\alpha_{s}^{2} \alpha$ mixed QCD-weak corrections.

As already emphasized at the beginning of this section we apply the large top-quark mass limit for the computation of the NLO QCD corrections to heavy Higgs production. Because we work above the top-quark pair production threshold we use this approximation somewhat beyond its range of validity. However, in Ref. [29] it was shown that the range of application can be extended to $m_{\phi}>2 m_{t}$ within rather small uncertainties $(\approx 10 \%)$, provided the higher order corrections are rescaled by an appropriate $\mathrm{K}$ factor. This procedure has been examined for the production of a heavy Higgs boson by gluon fusion in the SM, $p p \rightarrow \phi+X$. In Ref. [29] it was also shown that the main contribution to the QCD NLO corrections is due to soft and collinear gluon radiation which does not resolve the gluon-Higgs coupling. Because the QCD corrections to

$$
p p \rightarrow \phi_{2}, \phi_{3} \rightarrow t \bar{t}
$$

especially the factorizing ones, are very similar to those of Higgs production by gluon fusion in the SM, it is well motivated to assume the same behavior with respect to the effective gluonHiggs couplings that we use. The K-factor method applied to our calculation should therefore lead to reliable results. Compared to the case of inclusive SM Higgs production there is, however, a major difference: the interference with the QCD background. In the following we briefly describe the K-factor method adapted to the situation studied in this article. The total $t \bar{t}$ cross section is written as

$$
\sigma_{\mathrm{NLO}}=\sigma_{\mathrm{NLO}}^{\mathrm{QCDW}}+\sigma_{\mathrm{NLO}}^{\text {approx. }}
$$

where $\sigma_{\mathrm{NLO}}^{\mathrm{QCDW}}$ denotes the nonresonant $t \bar{t}$ cross section including the NLO QCD and weak corrections and $\sigma_{\mathrm{NLO}}^{\text {approx. }}$ is the contribution of the heavy Higgs bosons including the interference with the QCD background at NLO. We calculate it as follows 4 .

$$
\sigma_{\mathrm{NLO}}^{\text {approx. }}=\sum_{j=2,3}\left(\sigma_{\text {full }, j}^{(0)}+\sigma_{\text {full }, j, \mathrm{QCD}}^{(0)}+K_{j} \sigma_{\mathrm{eff}, j}^{(1)}+\sigma_{\mathrm{eff}, j, \mathrm{QCD}}^{(1)}\right)
$$

with

$$
K_{j}=\frac{\sigma_{\mathrm{full}, j}^{(0)}}{\sigma_{\mathrm{eff}, j}^{(0)}} .
$$

Here $\sigma_{\text {full }, j}^{(0)}$ denote the leading-order cross sections for

$$
p p \rightarrow \phi_{j} \rightarrow t \bar{t}
$$

and $\sigma_{\text {full, } j, \mathrm{QCD}}^{(0)}$ is the interference term of the $\phi_{j}$ production amplitude with the background at LO. The full top-quark mass dependence is kept in these two contributions. The terms $\sigma_{\mathrm{eff}, j}^{(1)}$ $\left(\sigma_{\mathrm{eff}, j}^{(0)}\right)$ and $\sigma_{\mathrm{eff}, j, \mathrm{QCD}}^{(1)}$ represent the NLO (LO) contributions to 66 and the interference with

\footnotetext{
${ }^{4}$ The formulas are presented here only for the inclusive cross section. However, they can also be applied to each individual bin of a distribution.
} 
the QCD background at NLO, respectively, in the effective theory. The K factor is not applied to the NLO interference term in (64). Using a K factor for this term analogous to Eq. 65),

$$
\tilde{K}_{j}=\frac{\sigma_{\text {full,j,QCD }}^{(0)}}{\sigma_{\mathrm{eff}, j, \mathrm{QCD}}^{(0)}},
$$

would lead to a singular behavior whenever $\sigma_{\mathrm{eff}, j, \mathrm{QCD}}^{(0)}$ vanishes. This can happen for differential cross sections, e.g. for the $M_{t \bar{t}}$ distribution.

\section{Results}

For the numerical evaluation of the (differential) cross section discussed in the previous section we use, apart from the 2HDM parameter scenarios introduced in Sec. 3, the following input values. The masses of the top quark (in the on-shell scheme) and of the SM-like Higgs boson $\phi_{1}$ are set to

$$
m_{t}=173.34 \mathrm{GeV}, \quad m_{1}=125 \mathrm{GeV} .
$$

We use the following values of the electromagnetic fine structure constant and the gauge boson masses:

$$
\alpha=\frac{1}{129}, \quad m_{W}=80.385 \mathrm{GeV}, \quad m_{Z}=91.1876 \mathrm{GeV} .
$$

We employ the PDF set CT10nlo [76] which provides also the value of the strong coupling at a scale $\mu$. To estimate the impact of uncalculated higher orders we set the renormalization scale $\mu_{r}$ equal to the factorization scale $\mu_{f}\left(\mu_{f}=\mu_{r} \equiv \mu\right)$ and vary $\mu$ by a factor 2 up and down. As the central scale we use

$$
\mu_{0}=\frac{m_{2}+m_{3}}{4} .
$$

This choice is motivated by the choice $\mu_{0}=m_{H} / 2$ in the SM case; see, for instance, [77].

In the following we present results for proton-proton collisions at a hadronic center-of-mass energy of $\sqrt{s}=13 \mathrm{TeV}$. The $t \bar{t}$ cross section differential in the top-quark pair invariant mass is displayed in Figs. $10-12$ for scenarios 1-3. The ratios in the lower panes of these figures show that the corrections with respect to the leading-order Higgs contribution are sizeable but the overall effect of resonant $\phi_{2}, \phi_{3}$ production is rather small: In the $M_{t \bar{t}}$ distribution it ranges from below $5 \%$ in the $C P$-violating case with significantly different $\phi_{2}, \phi_{3}$ masses (scenario 3 ) to about $6 \%$ in the nearly mass-degenerate $C P$-conserving case (scenario 1 ). The effects on the inclusive $t \bar{t}$ cross section are even smaller, as can be seen in Table 7 . As a general feature we observe that in all three scenarios the QCD corrections lead to a positive shift in the vicinity of the resonances with respect to the leading-order results. The potential cancellation due to the peak-dip structure, when the resonance region is integrated over, is thus reduced at nextto-leading order.

Because the validity of the predictions is restricted in the pole approximation to the resonant region, we apply $M_{t \bar{t}}$ cuts around the resonance which are indicated as hatched regions in the 
lower plots in Figs. $10-12$. In order to enhance the signal we divide the $M_{t \bar{t}}$ cuts into two regions. One region is associated with the peak

$$
m^{*}-150 \mathrm{GeV} \leq M_{t \bar{t}} \leq m^{*}
$$

and the other one with the dip

$$
m^{*} \leq M_{t \bar{t}} \leq m^{*}+150 \mathrm{GeV}
$$

where $m^{*}$ is defined as the smallest value of $M_{t \bar{t}}$ where the NLO ratio (red curve in Figs. 10 12) crosses 1 . These cuts enhance the signal-to-background ratio because they prevent the partial cancellation due to the peak-dip structure. As already mentioned in the introduction experimental analyses may use a sliding $M_{t \bar{t}}$ window.

Table 7: Inclusive $t \bar{t}$ production cross sections for $p p$ collisions at the hadronic center-of-mass energy of $\sqrt{s}=13 \mathrm{TeV}$ in different type-II 2HDM scenarios. $\sigma_{\text {QCDW }}$ denotes the cross section for $p p \rightarrow t \bar{t}$ including NLO QCD and weak corrections, but without contributions from $\phi_{2}, \phi_{3}$. Note that due to our choice of the renormalization and factorization scale $\mu=\mu_{0}=\left(m_{2}+m_{3}\right) / 4$ the cross section $\sigma_{\mathrm{QCDW}}$ depends on the scenario. $\sigma_{2 \mathrm{HDM}}$ denotes the cross section for $p p \rightarrow \phi_{2,3} \rightarrow t \bar{t}$ including the interference with the QCD background at NLO QCD. The superscripts (subscripts) correspond to $\mu=\mu_{0} / 2\left(\mu=2 \mu_{0}\right)$. The scale variation changes the ratio by less than \pm 0.001 and is not displayed in the table.

\begin{tabular}{l|lll} 
& Scenario 1 & Scenario 2 & Scenario 3 \\
\hline$\sigma_{\mathrm{QCDW}}[\mathrm{pb}]$ & $643.22_{-77.71}^{+81.23}$ & $624.25_{-76.19}^{+80.98}$ & $619.56_{-75.72}^{+81.05}$ \\
$\sigma_{2 \mathrm{HDM}}[\mathrm{pb}]$ & $13.59_{-1.64}^{+1.85}$ & $7.4_{-0.78}^{+0.77}$ & $7.21_{-0.77}^{+0.81}$ \\
\hline$\sigma_{2 \mathrm{HDM}} / \sigma_{\mathrm{QCDW}}$ & 0.021 & 0.012 & 0.012
\end{tabular}

We investigate the sensitivity of such an analysis (Fig. 13) by calculating the number of heavy Higgs signal and background events for the dileptonic $t \bar{t}$ final states $(l=e, \mu)$

$$
N_{s, b}^{l l}=\mathcal{L} \sigma_{s, b} \operatorname{Br}(t \bar{t} \rightarrow 2 l) \epsilon_{l l}
$$

and for the lepton plus jets final states $(l=e, \mu)$

$$
N_{s, b}^{l j}=\mathcal{L} \sigma_{s, b} \operatorname{Br}(t \bar{t} \rightarrow l+\text { jets }) \epsilon_{l j}
$$

assuming the branching ratios

$$
\operatorname{Br}(t \bar{t} \rightarrow 2 l)=\frac{4}{81}, \quad \operatorname{Br}(t \bar{t} \rightarrow l+\text { jets })=\frac{24}{81},
$$

an integrated luminosity of $\mathcal{L}=100 \mathrm{fb}^{-1}$, and selection efficiencies [78, 79] $\epsilon_{l l}=0.22$ and $\epsilon_{l j}=0.12$. We assume that the observed number of events $N_{\text {obs }}$ is given by $N_{\text {obs }}=N_{s}+N_{b}$, 
where $N_{b}$ is the number of background events without the heavy Higgs bosons and $N_{\text {obs }}$ is the total number of events including those from resonant $\phi_{2}, \phi_{3}$ production including the HiggsQCD interference. These events are calculated for $M_{t \bar{t}}$ windows of different width $(20,80$ and $140 \mathrm{GeV}$ ). We consider for the lower boundary of the $M_{t \bar{t}}$ windows the range 340-800 $\mathrm{GeV}$ in steps of $20 \mathrm{GeV}$. We determine from the number of signal and background events the significance $Z_{\mathrm{PL}}[80,81]$ based on the profile likelihood method for each $M_{t \bar{t}}$ window. In the calculation of $Z_{\mathrm{PL}}$ we include an uncertainty on the background of $1 \%$ (upper pane in Fig. 13) and $5 \%$ (lower pane in Fig. 13, respectively. Only the significance of the dileptonic channel $Z_{\mathrm{PL}}^{l l}$ is shown in Fig. 13 . The results for the significance of the lepton plus jets channel $Z_{\mathrm{PL}}^{l j}$ are very similar. Figure 13 shows that the significance increases if the $M_{t \bar{t}}$ window becomes narrower. In wider $M_{t \bar{t}}$ windows peak values for the signal-to-background ratio are averaged out whereas for smaller windows peaks in the signal-to-background ratio show up as peaks in the significance. Because the experimental resolution is limited we show in Fig. 13 also results for realistic $M_{t \bar{t}}$ window widths of $80 \mathrm{GeV}$ and $140 \mathrm{GeV}$. Comparing the upper and lower plot in Fig. 13 where we have assumed a $1 \%$ and 5\% background uncertainty, respectively, we observe that increasing the assumed uncertainty by a factor of 5 leads to a reduction of the significance by a factor of $1 / 5$. This is due to

$$
\lim _{N_{b} \rightarrow \infty} Z_{\mathrm{PL}}=\frac{\sqrt{2(\eta-\ln (1+\eta))}}{\varepsilon},
$$

where $\eta$ is the signal-to-background ratio and $\varepsilon$ is the relative background uncertainty. This emphasizes the importance of reducing background uncertainties.

In Table 8 we show numerical values of the significances $Z_{\mathrm{PL}}^{l l}$ and $Z_{\mathrm{PL}}^{l j}$ for all three scenarios using $M_{t \bar{t}}$ windows of $80 \mathrm{GeV}$ width. The values for $Z_{\mathrm{PL}, 1 \%}^{l l}$ and $Z_{\mathrm{PL}, 5 \%}^{l l}$ for scenario 1 can be directly compared to Fig. 13 . The lepton plus jets decay channel has considerably higher event numbers than the dileptonic channel because of the higher branching ratio. However, the significance in both channels is almost the same. This is because we fixed the relative background uncertainty and the signal-to-background ratio is independent of the channel. A further increase of the luminosity would thus only marginally increase the significance, given a constant relative uncertainty of the background [see also Eq. (75)]. We have also calculated the significances based on the $p$-value of the Poisson distribution from the number of observed events $N_{\text {obs }}$ and background events $N_{b}$ ignoring the uncertainty. The resulting values for these significances become unreasonably high and cannot be trusted.

In Fig. 14 we show for the $M_{t \bar{t}}$ distribution a comparison of $8 \mathrm{TeV}$ data from the CMS experiment [27] with theoretical predictions obtained within scenario 1 . One can see that the current experimental and theoretical accuracy is insufficient to establish or exclude scenario 1.

\footnotetext{
${ }^{5}$ According to Table 7 the theory uncertainty of the background calculated at NLO is about $13 \%$. The uncertainties of the NLO background cross sections within the $M_{t \bar{t}}$ windows used in Fig. 13 are roughly the same. These uncertainties would reduce the significance $Z_{\mathrm{PL}}$ to a value below 1 . However, we expect that the uncertainty of $13 \%$ can be reduced by using higher order QCD predictions of the background, in particular the next-to-next-to-leading order QCD corrections to $t \bar{t}$ production [82,83] and by using a sideband analysis to determine the background in the signal region.
} 
Table 8: Number of events $(N)$ and significance $(Z)$ for the dileptonic (superscript $l l$ ) and lepton plus jets (superscript $l j$ ) channels $(l=e, \mu)$ at $\sqrt{s}=13 \mathrm{TeV}$ assuming an integrated luminosity of $\mathcal{L}=100 \mathrm{fb}^{-1}$ at the LHC.

\begin{tabular}{l|l|l|l|l|l|l} 
& \multicolumn{2}{|c|}{ scenario 1 } & \multicolumn{2}{c|}{ scenario 2 } & \multicolumn{2}{c}{ scenario 3 } \\
\hline$M_{t \bar{t}}$ cut $(\mathrm{GeV})$ & $460-540$ & $540-620$ & $480-560$ & $560-640$ & $420-500$ & $500-580$ \\
\hline$N_{b}^{l l}$ & 153517 & 90050 & 130718 & 76566 & 190486 & 113261 \\
$N_{\mathrm{obs}}^{l l}$ & 160536 & 88250 & 135112 & 76357 & 196907 & 111712 \\
\hline$Z_{\mathrm{PL}, 1 \%}^{l l}$ & 4.4 & 1.9 & 3.2 & 0.3 & 3.3 & 1.3 \\
$Z_{\mathrm{PL}, 5 \%}^{l l}$ & 0.9 & 0.4 & 0.7 & 0.1 & 0.7 & 0.3 \\
\hline \hline$N_{b}^{l j}$ & 502422 & 294711 & 427805 & 250582 & 623410 & 370675 \\
$N_{\mathrm{obs}}^{l j}$ & 525393 & 288819 & 442186 & 249897 & 644423 & 365603 \\
\hline$Z_{\mathrm{PL}, 1 \%}^{l j}$ & 4.5 & 2 & 3.3 & 0.3 & 3.3 & 1.4 \\
$Z_{\mathrm{PL}, 5 \%}^{l j}$ & 0.9 & 0.4 & 0.7 & 0.1 & 0.7 & 0.3
\end{tabular}

Table 9: $M_{t \bar{t}}$ windows used in the computation of the $y_{t}$ and $\cos \theta_{C S}$ distributions.

\begin{tabular}{l|l|l} 
& Lower $M_{t \bar{t}}$ window & Upper $M_{t \bar{t}}$ window \\
\hline Scenario 1 & $390 \mathrm{GeV} \leq M_{t \bar{t}} \leq 540 \mathrm{GeV}$ & $540 \mathrm{GeV} \leq M_{t \bar{t}} \leq 690 \mathrm{GeV}$ \\
Scenario 2 & $410 \mathrm{GeV} \leq M_{t \bar{t}} \leq 560 \mathrm{GeV}$ & $560 \mathrm{GeV} \leq M_{t \bar{t}} \leq 710 \mathrm{GeV}$ \\
Scenario 3 & $350 \mathrm{GeV} \leq M_{t \bar{t}} \leq 500 \mathrm{GeV}$ & $500 \mathrm{GeV} \leq M_{t \bar{t}} \leq 650 \mathrm{GeV}$
\end{tabular}




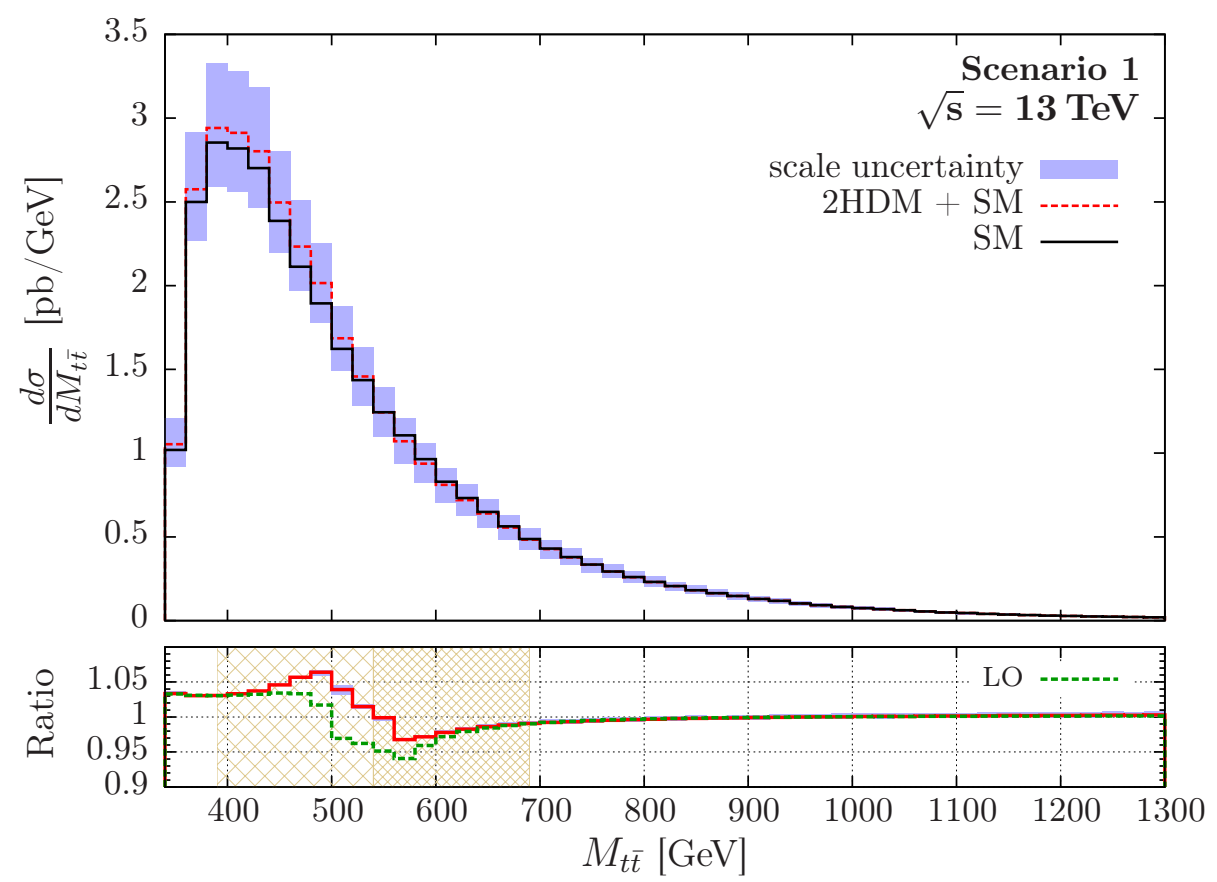

Figure 10: Invariant mass distribution $M_{t \bar{t}}$ of $t \bar{t}$ for scenario 1 at NLO. The upper pane shows the SM contribution (solid black) which includes NLO QCD and weak corrections. The dashed red line shows the sum of SM and 2HDM contributions at NLO QCD. The shaded blue area represents the scale uncertainty when varying $\mu_{R}=\mu_{F}=\mu_{0}$ by a factor of 2 or $1 / 2$, respectively, in the combined SM $+2 \mathrm{HDM}$ result. The lower pane shows the sum of SM and 2HDM contributions normalized to the SM contribution at NLO (solid red) and LO (dashed green). The scale uncertainty (shaded blue) of the ratio is very small and is invisible for most of the bins in this plot. The hatched regions in the lower pane indicate the range of the $M_{t \bar{t}}$ cuts.

Furthermore, the limits on the cross section for a spin-zero resonance stated in Ref. [27] were derived without taking interference effects between signal and background into account. There is a more recent ATLAS analysis on resonances in the $M_{t \bar{t}}$ distribution, Ref. [28], but it is also not sensitive enough to establish or exclude our scenarios. 


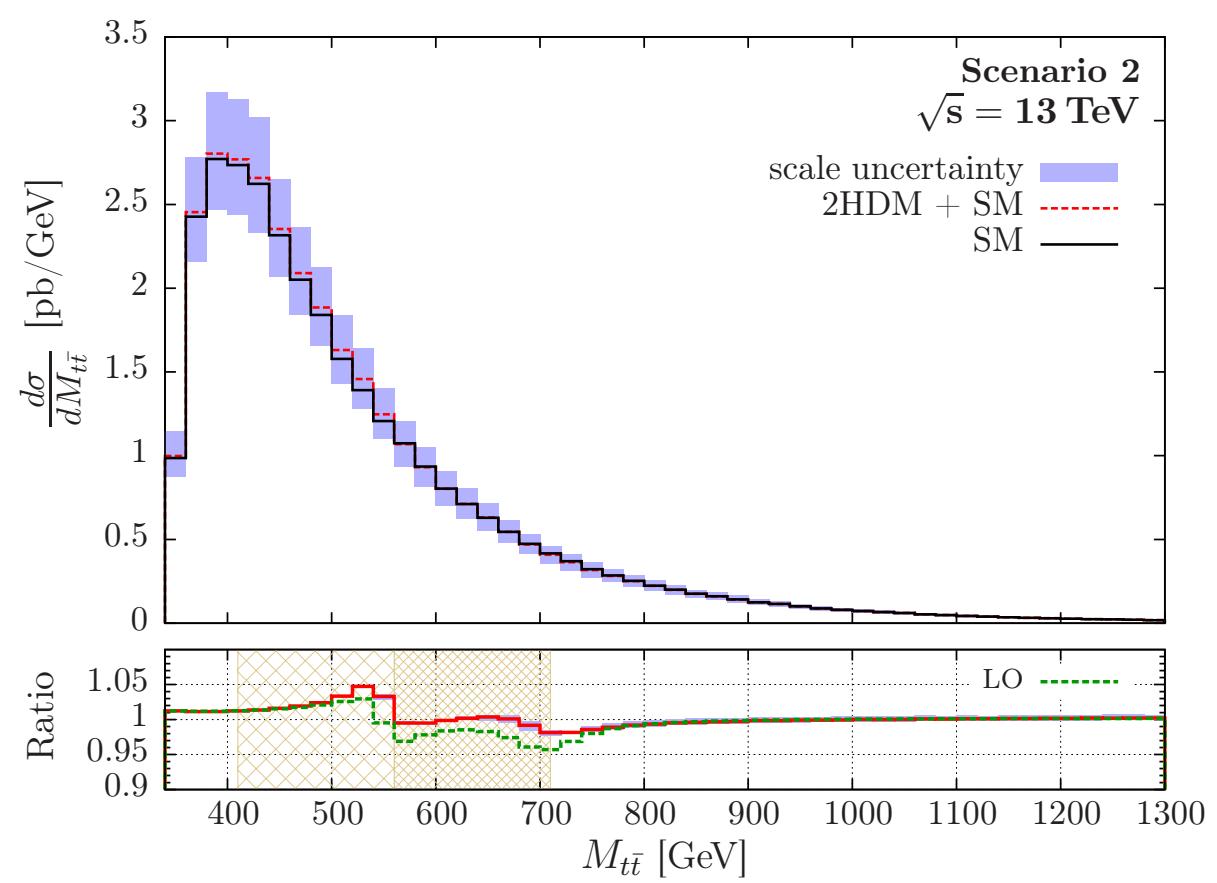

Figure 11: Same as Fig. 10, but for scenario 2.

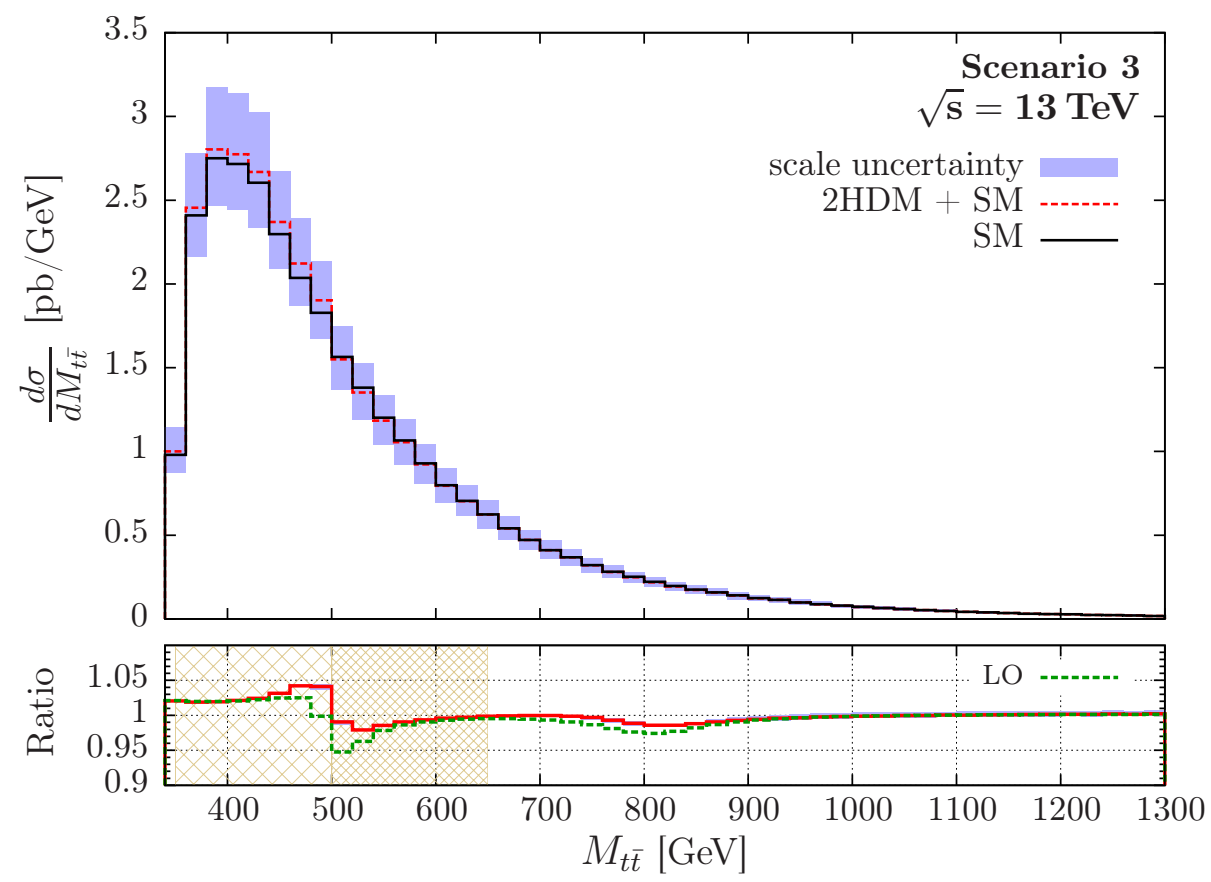

Figure 12: Same as Fig. 10, but for scenario 3. 

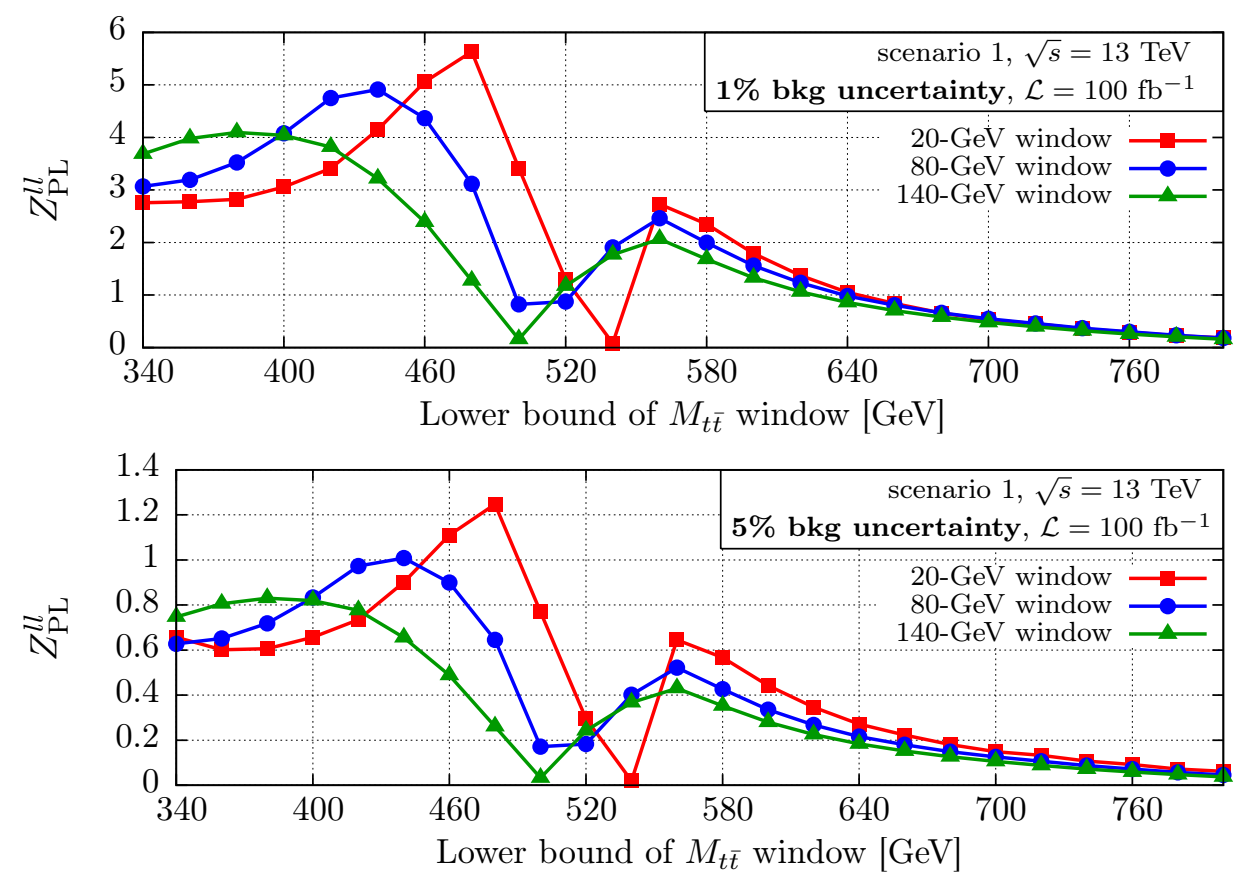

Figure 13: Significance $Z_{\mathrm{PL}}^{l l}$ for different cuts on $M_{t \bar{t}}$. The solid red (dashed blue, dotted green) curve shows the behavior for $M_{t \bar{t}}$ windows of $20 \mathrm{GeV}(80 \mathrm{GeV}, 140 \mathrm{GeV})$ width. On the abscissa the lower boundaries of the $M_{t \bar{t}}$ windows are given. The upper (lower) pane shows the results for $Z_{\mathrm{PL}}^{l l}$ where a $1 \%(5 \%)$ uncertainty on the background is assumed. 


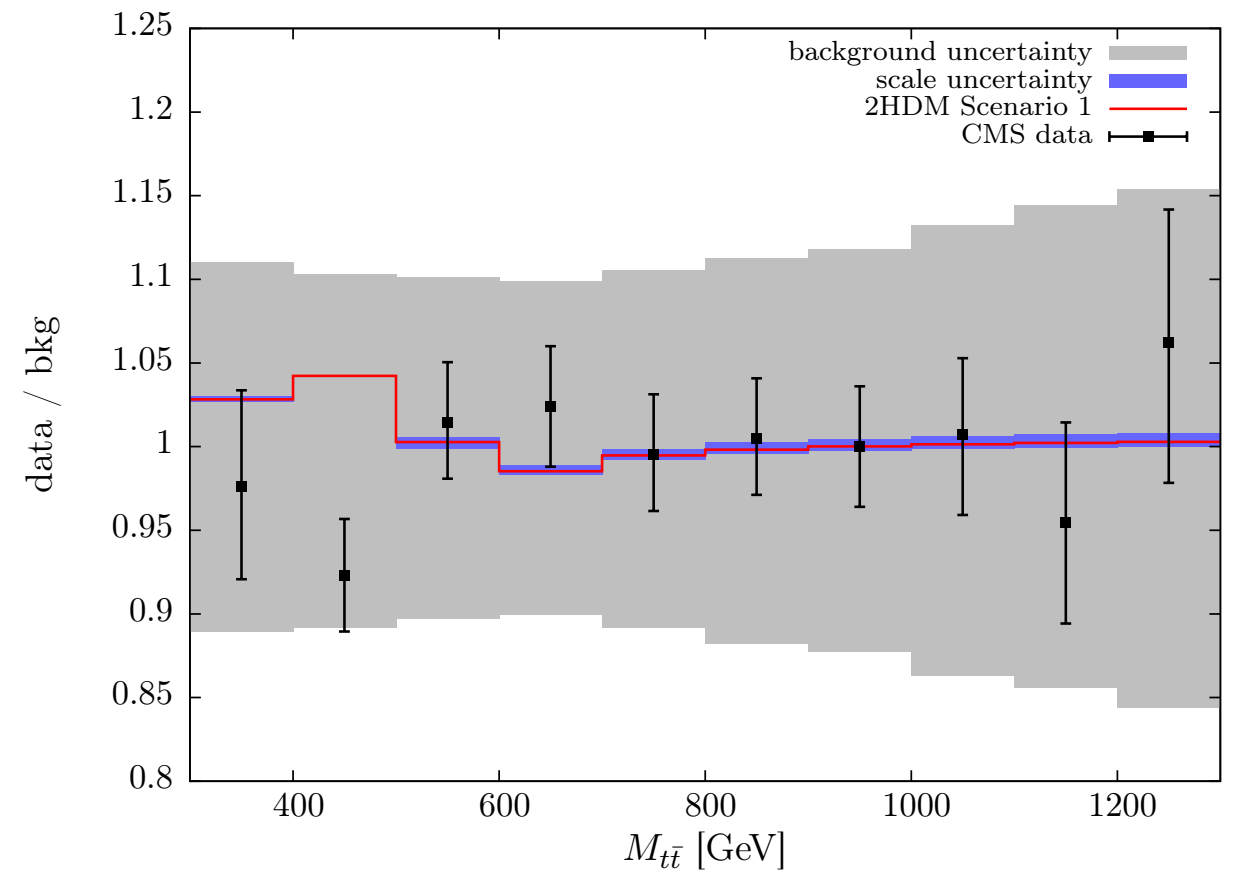

Figure 14: $M_{t \bar{t}}$ distribution for $p p \rightarrow t \bar{t} X$ at the LHC $(8 \mathrm{TeV})$. The black data points were extracted from Fig. 4(b) of Ref. [27] using the tool EasyNData [84]. For error bars not visible in the original plot the radius of the black circle representing the respective data point is used as the error bar length. The shaded grey area was also extracted from Fig. 4(b) of Ref. [27]. It displays the theoretical uncertainty of the background Monte Carlo prediction. The solid red curve in this plot shows our result for scenario 1 including NLO QCD corrections. The shaded blue area represents the scale uncertainty due to the variation of $\mu_{R}=\mu_{F}=\mu_{0}$ by a factor of 2 or $1 / 2$, respectively. 


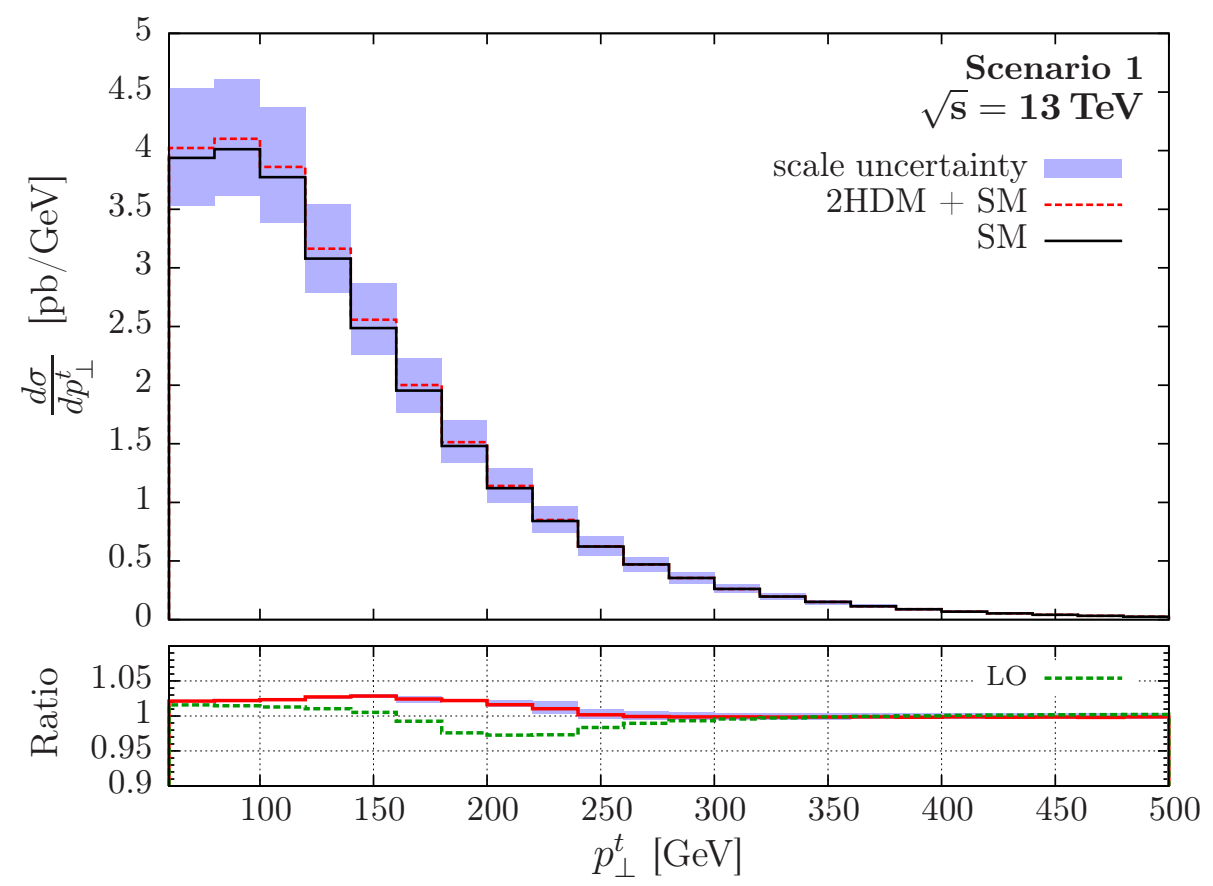

Figure 15: Transverse momentum distribution of the top quark $p_{\perp}^{t}$ in scenario 1 of the 2HDM at NLO. The upper pane shows the SM contribution (solid black) which includes NLO QCD and weak corrections. The dashed red line displays the sum of the SM contribution and 2HDM contribution at NLO QCD. The shaded blue area represents the scale uncertainty when varying $\mu_{R}=\mu_{F}=\mu_{0}$ by a factor of 2 or $1 / 2$, respectively, in the combined SM $+2 \mathrm{HDM}$ result. The plot in the lower pane shows the sum of SM and 2HDM contributions normalized to the SM contribution at NLO (solid red) and LO (dashed green). The scale uncertainty (shaded blue) of the ratio is very small and is invisible for most of the bins in this plot.

The distributions of the top-quark transverse momentum $p_{\perp}^{t}$ are displayed in Figs. $15-17$ for scenarios 1-3, respectively. These distributions are computed without $M_{t \bar{t}}$ cuts. A cut on the $M_{t \bar{t}}$ value in the $p_{\perp}^{t}$ distribution restricts the Born contribution and the virtual corrections to values $p_{\perp}^{t} \leq \sqrt{\left(M_{t \bar{t}}^{\text {cut }}\right)^{2} / 4-m_{t}}$. As a consequence the results are only leading order in the strong coupling constant for $p_{\perp}^{t}>\sqrt{\left(M_{t \bar{t}}^{\text {cut }}\right)^{2} / 4-m_{t}}$. As in the case of the $M_{t \bar{t}}$ distribution the QCD corrections to the $2 \mathrm{HDM}$ scenarios are mainly positive such that the ratios of the $p_{\perp}^{t}$ distributions in all three scenarios stay above one. However, the deviations from one are small. As a remnant of the resonance structure an enhancement around $p_{\perp}^{t} \approx m_{i} / 2$ can be observed.

In the following we present, for the LHC (13 TeV), a number of distributions for $t \bar{t}$ events obeying the $M_{t \bar{t}}$ cuts given in Table 9. The top-quark rapidity distributions in the laboratory frame, $y_{t}$, are displayed in Figs. $18-20$ for scenarios 1-3, respectively. As expected the $y_{t}$ distributions - and the rapidity distributions of the $\bar{t}$ quarks, which are not shown-are (within small statistical fluctuations) symmetric around $y_{t}=0$. The impact of the heavy Higgs bosons 


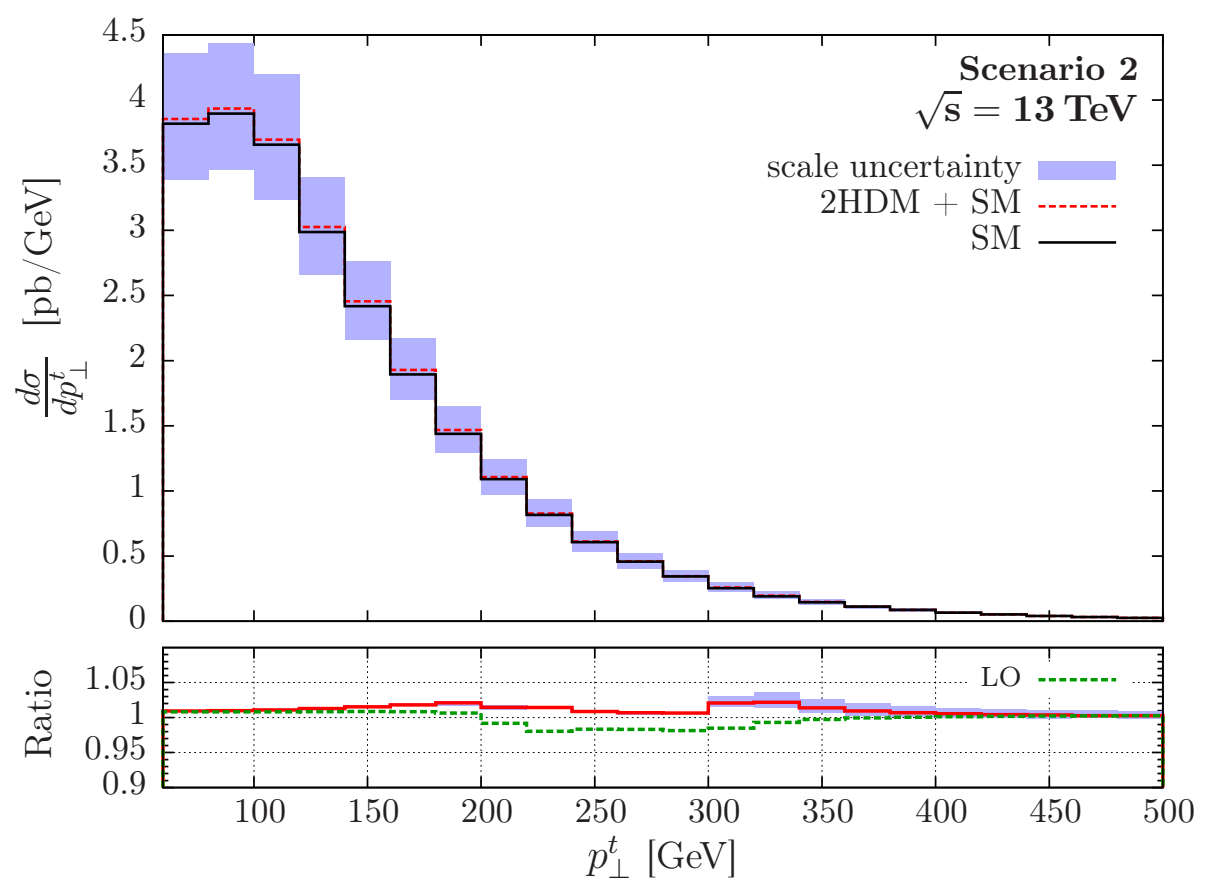

Figure 16: Same as Fig. 15, but for scenario 2.

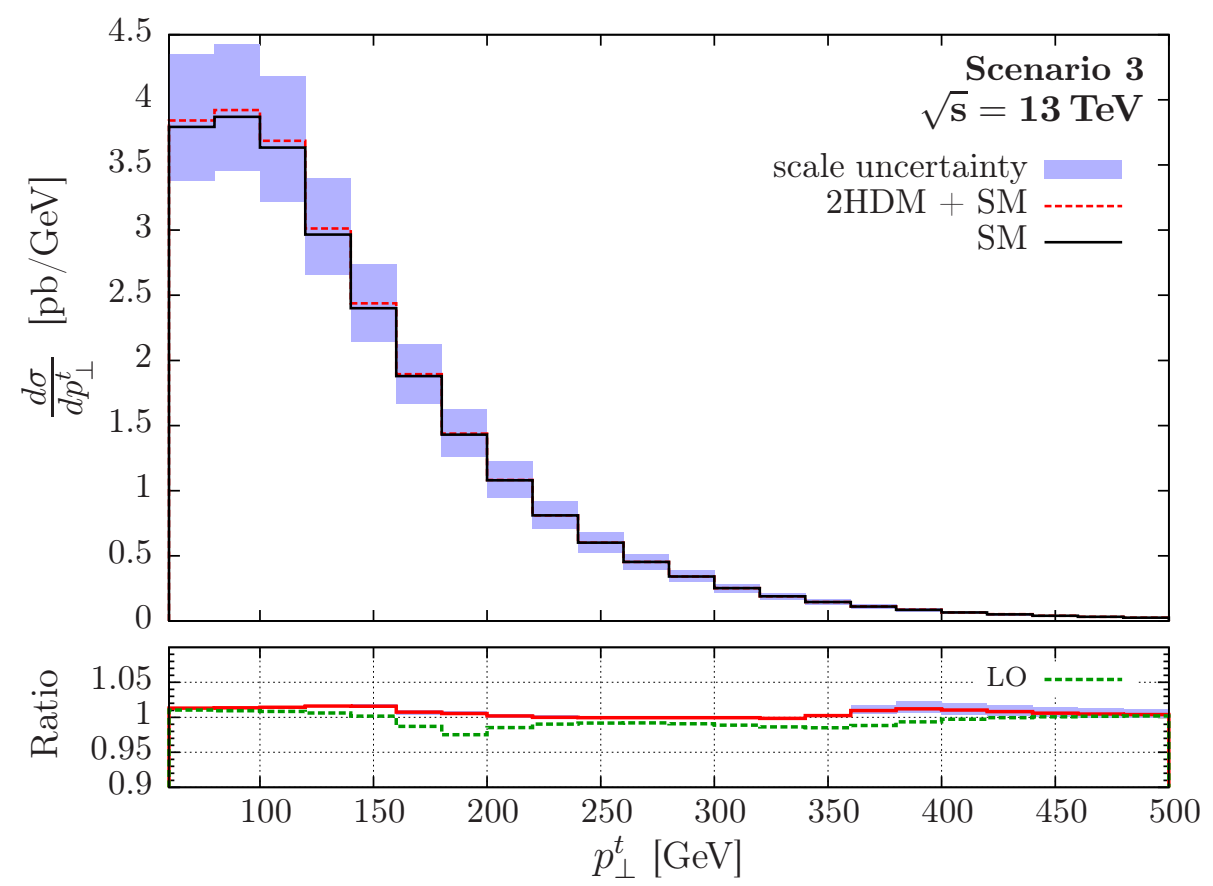

Figure 17: Same as Fig. 15, but for scenario 3. 
is rather small, it is maximally $\sim 5 \%$ in the central region for scenario 1 in the low $M_{t \bar{t}}$ window. Top-quark pair production by $q \bar{q}$ annihilation and (anti)quark gluon fusion at NLO QCD induces a small asymmetry in the distribution of the moduli difference $\Delta|y|=\left|y_{t}\right|-\left|y_{\bar{t}}\right|$. The inclusive $t \bar{t}$ charge asymmetry $A_{C}=[N(\Delta|y|>0)-N(\Delta|y|<0)] / N_{\text {tot }}$ at $13 \mathrm{TeV}$ (integrated over $\left.M_{t \bar{t}}\right)$ is $A_{C}=0.75(5) \times 10^{-2}$ at NLO QCD including electroweak corrections. The heavy Higgs boson contributions, which are symmetric with respect to the interchange of $t$ and $\bar{t}$, do not change this result.

The distributions of the cosine of the Collins-Soper angle [30] $\cos \theta_{C S}$ are shown in Figs. 21. 23 for scenarios $1-3$, respectively. We recall that $\theta_{C S}$ is similar to the angle between the top quark and the direction of one of the beams in the $t \bar{t}$ zero-momentum frame (ZMF) - in fact, at leading order they are identical-, but $\theta_{C S}$ is less affected by initial state radiation. Let us denote the momenta of the two proton beams in the $t \bar{t} \mathrm{ZMF}$ by $p_{1}$ and $p_{2}$. If the transverse momentum of the $t \bar{t}$ pair is nonzero the three-momenta $\mathbf{p}_{1}$ and $\mathbf{p}_{2}$ are not collinear, and $\theta_{C S}$ is defined to be the angle between the top-quark direction in the $t \bar{t} \mathrm{ZMF}$ and the bisecting line between $\mathbf{p}_{1}$ and $-\mathbf{p}_{2}$. For the production of a spin-zero resonance and its decay to $t \bar{t}$ the $\cos \theta_{C S}$ distribution is flat at LO. For the background at LO QCD, one gets for $q \bar{q} \rightarrow t \bar{t}$ a distribution proportional to $\hat{s}\left(1+\cos ^{2} \theta_{C S}\right)+4 m_{t}^{2}\left(1-\cos ^{2} \theta_{C S}\right)$. For $g g \rightarrow t \bar{t}$, where all partial waves are present, the $\cos \theta_{C S}$ distribution is more complicated, but is also minimal in the central region and maximal in the forward and backward region. The background distributions computed at NLO QCD including weak corrections, which are displayed in Figs. 21, 23, corroborate this behavior. Adding the contributions of the heavy Higgs bosons and the interference with the background changes the shape of the $\cos \theta_{C S}$ distributions only moderately. The effect is largest in the low $M_{t \bar{t}}$ window of scenario 1, where the distribution changes by $\sim 6 \%$ in the central region.

Finally, we have also calculated the above distributions for $14 \mathrm{TeV}$. We do not display them here because these results are similar to those for $13 \mathrm{TeV}$ and do not bear significant new insights. 


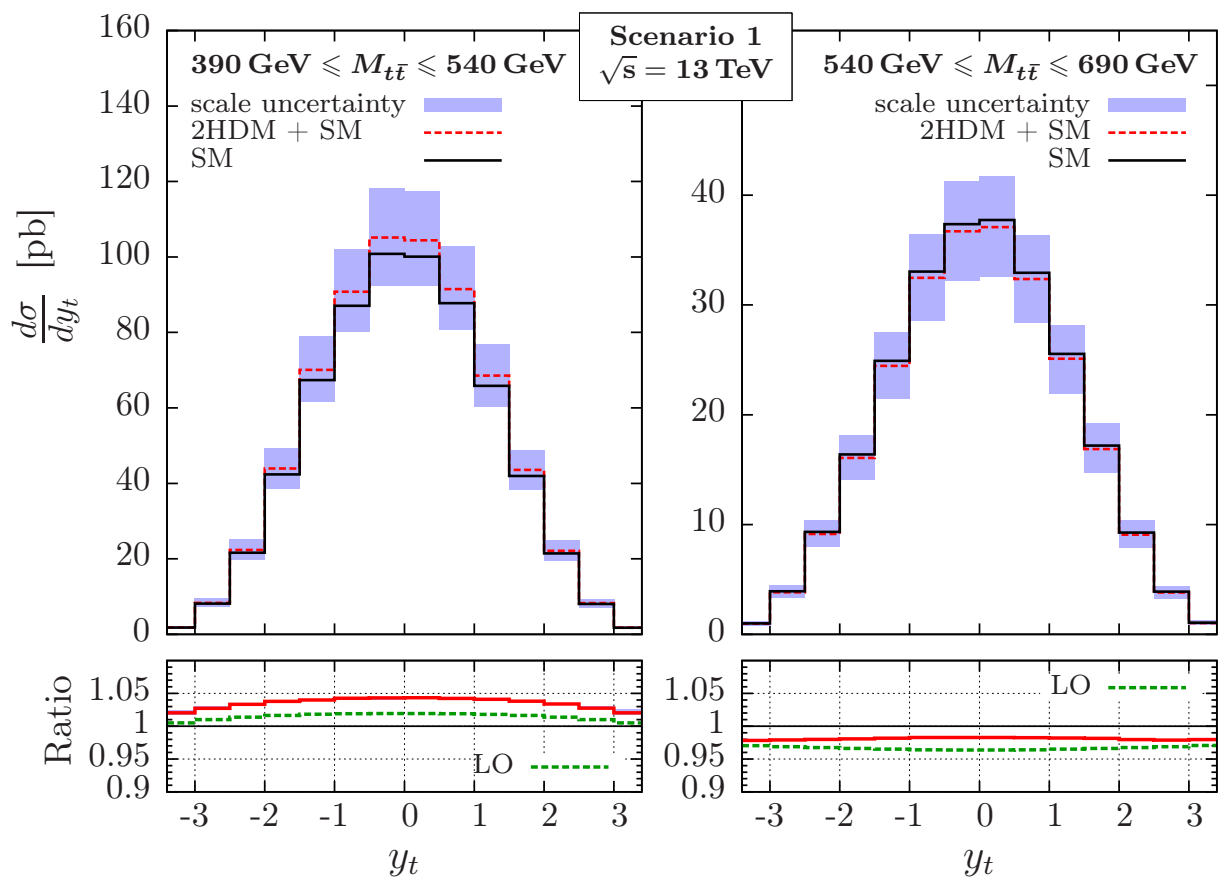

Figure 18: Top-quark rapidity distribution $y_{t}$ for scenario 1 of the $2 \mathrm{HDM}$ at NLO. The plots in the upper panes show the SM contribution (solid black) which includes NLO QCD and weak corrections. The dashed red line shows the sum of the SM contribution and 2HDM contribution at NLO QCD. The shaded blue area represents the scale uncertainty when varying $\mu_{R}=\mu_{F}=\mu_{0}$ by a factor of 2 or $1 / 2$, respectively, in the combined SM + 2HDM result. The plots in the lower panes show the sum of SM and 2HDM contributions normalized to the SM contribution at NLO (solid red) and LO (dashed green). The scale uncertainties (shaded blue) of the ratios are very small and are invisible in these plots. The plots on the left- and right-hand side correspond to different $M_{t \bar{t}}$ windows. 


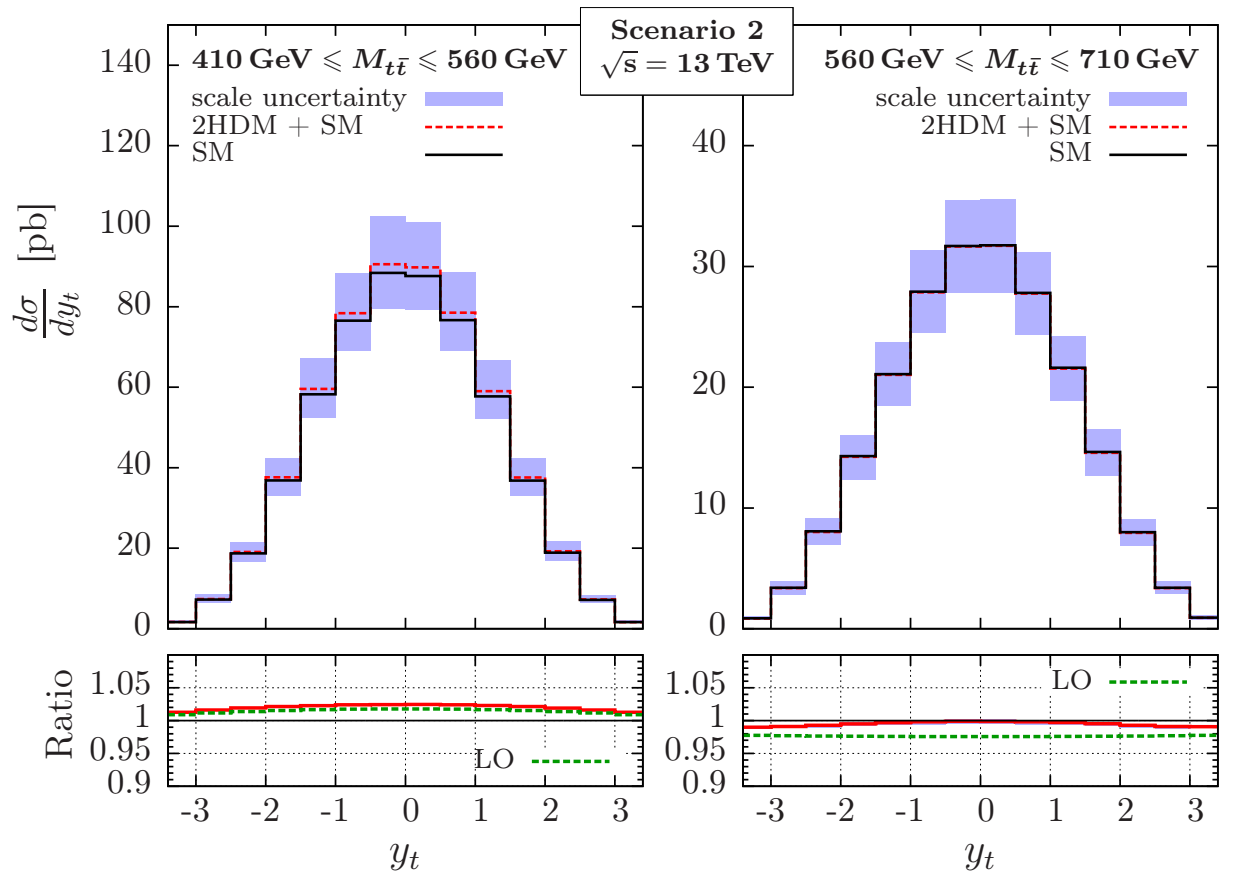

Figure 19: Same as Fig. 18 , but for scenario 2 and different $M_{t \bar{t}}$ windows.

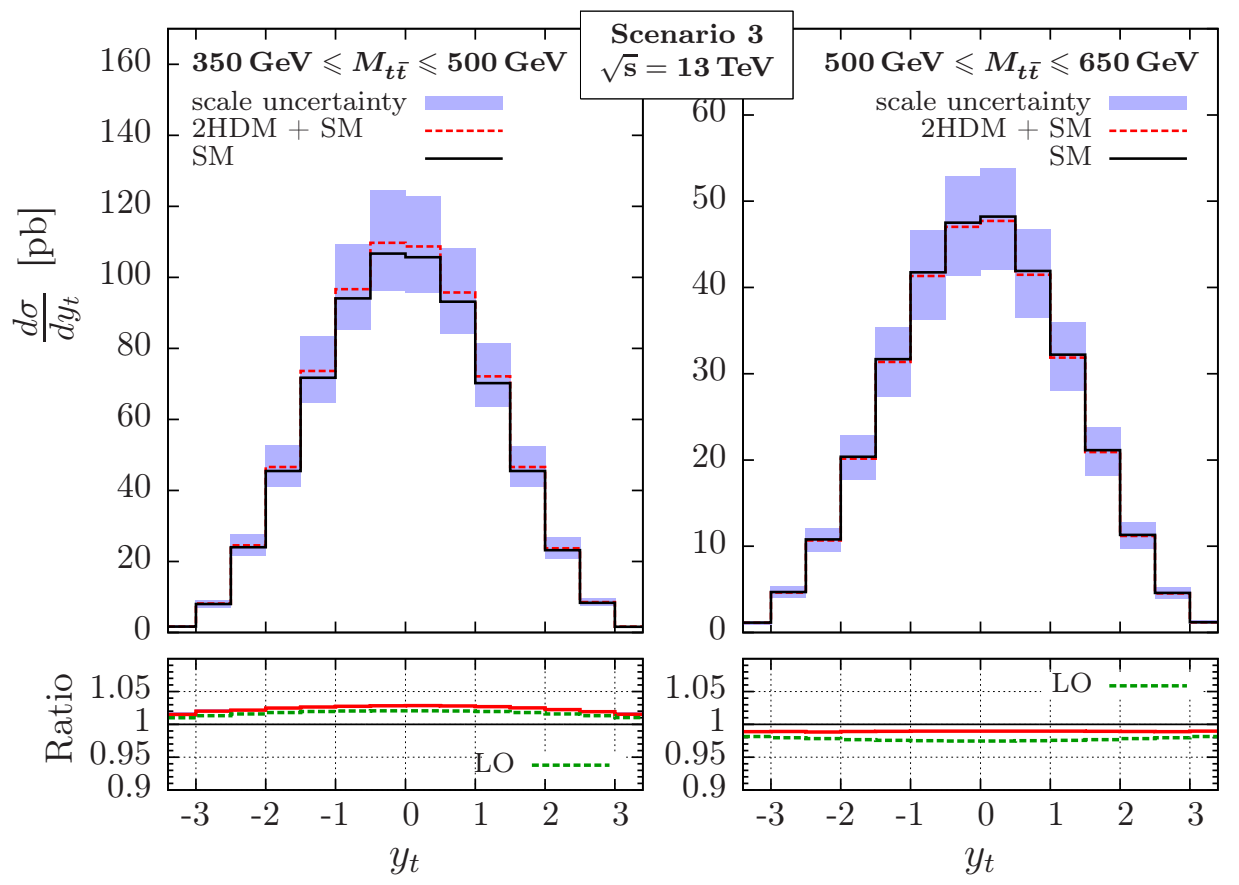

Figure 20: Same as Fig. 18 , but for scenario 3 and different $M_{t \bar{t}}$ windows. 


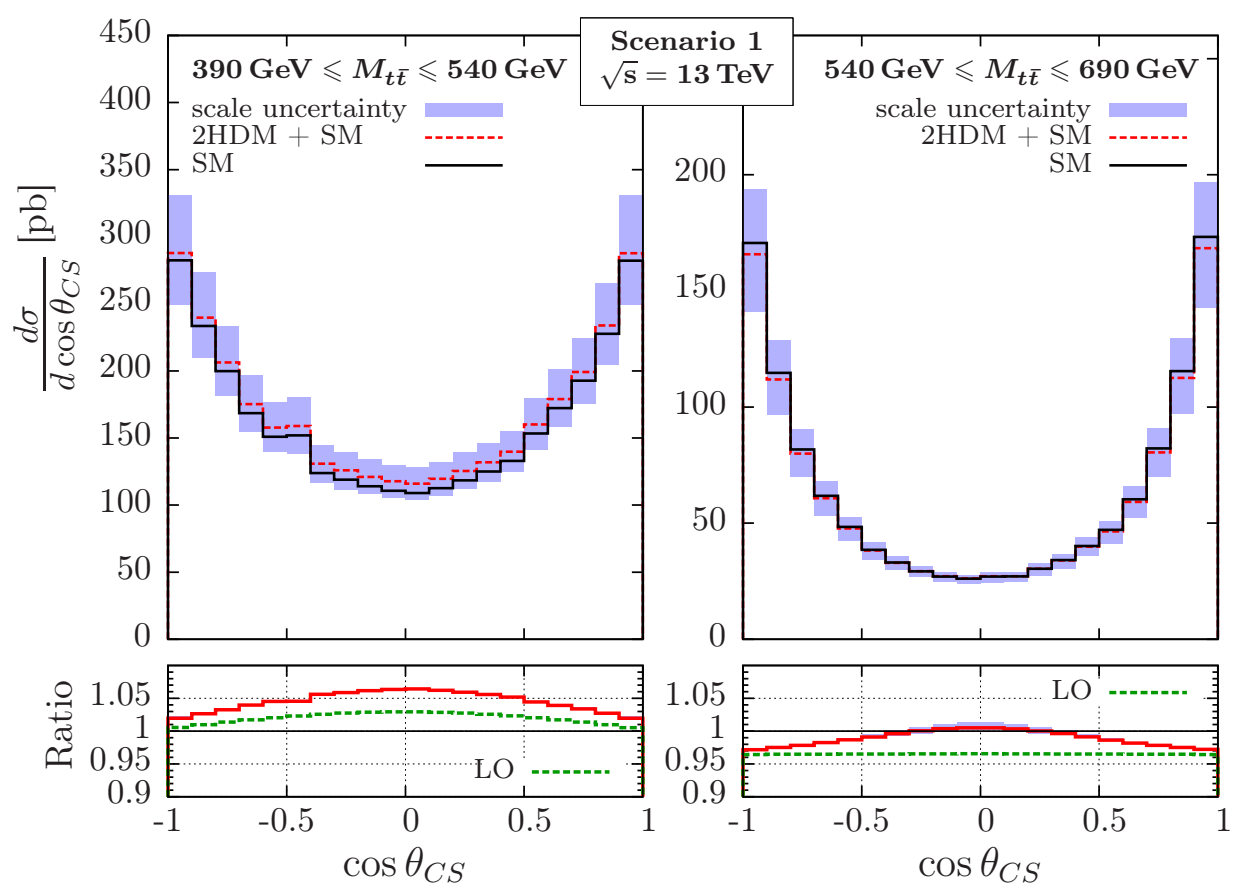

Figure 21: Distribution of the cosine of the Collins-Soper angle, $\cos \theta_{C S}$, for the top quark in scenario 1 of the $2 \mathrm{HDM}$ at NLO. The plots in the upper panes show the SM contribution (solid black) which includes NLO QCD and weak corrections. The dashed red line displays the sum of the SM contribution and 2HDM contribution at NLO QCD. The shaded blue area represents the scale uncertainty when varying $\mu_{R}=\mu_{F}=\mu_{0}$ by a factor of 2 or $1 / 2$, respectively, in the combined SM $+2 \mathrm{HDM}$ result. The plots in the lower panes show the sum of SM and 2HDM contributions normalized to the SM contribution at NLO (solid red) and LO (dashed green). The scale uncertainties (shaded blue) of the ratios are very small and are invisible for most of the bins in these plots. The plots on the left- and right-hand side correspond to different $M_{t \bar{t}}$ windows. 


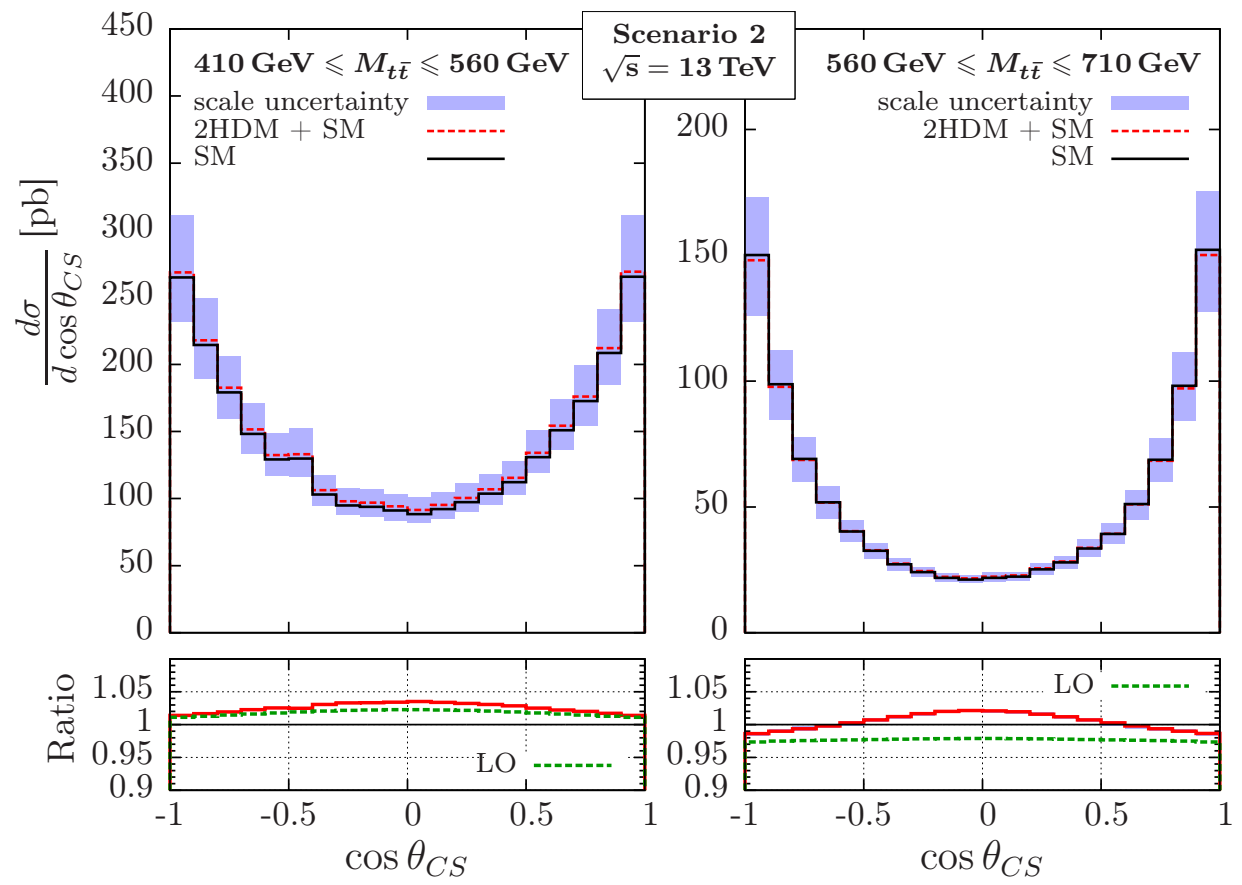

Figure 22: Same as Fig. 21, but for scenario 2 and different $M_{t \bar{t}}$ windows.

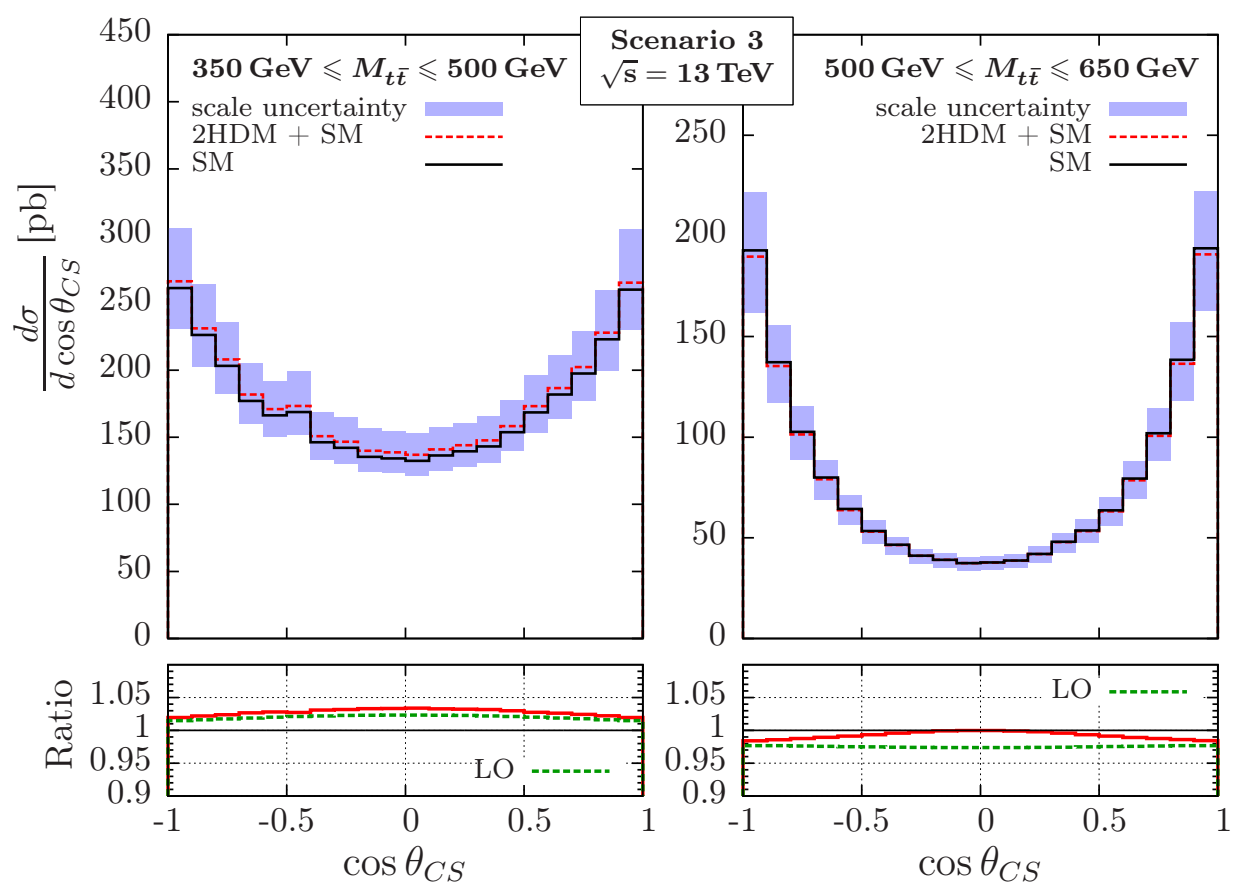

Figure 23: Same as Fig. 21, but for scenario 3 and different $M_{t \bar{t}}$ windows. 


\section{Summary and conclusions}

This paper is based on the hypothesis that in addition to the $125 \mathrm{GeV}$ Higgs boson additional heavy neutral Higgs bosons with unsuppressed Yukawa couplings to top quarks exist, which can be resonantly produced at the LHC in the $t \bar{t}$ channel. The analysis of $t \bar{t}$ data at the LHC, which so far has been rather rudimentary with regard to this possibility, requires precise theoretical predictions. We have therefore investigated effects of heavy, neutral Higgs boson resonances in $t \bar{t}$ production at the LHC at next-to-leading order in the strong coupling. A new ingredient of our analysis is the incorporation of the interferences of the heavy Higgs production amplitudes with the respective nonresonant QCD $t \bar{t}$ amplitudes at NLO QCD. The NLO corrections to heavy Higgs boson production and the Higgs-QCD interference were computed in the large $m_{t}$ limit with an effective $\mathrm{K}$-factor rescaling. The nonresonant $t \bar{t}$ background was incorporated at NLO QCD including weak-interaction corrections.

Our NLO QCD computation of heavy Higgs production and decay into $t \bar{t}$ and the interference with the nonresonant $t \bar{t}$ amplitude is in principle rather model independent. However, essential ingredients in our analysis are the total decay widths of the heavy Higgs bosons, which must be computed in a concrete model in order to maintain the unitarity of the $S$-matrix. To be specific, we used the type-II two-Higgs-doublet model where three neutral Higgs bosons appear in the physical particle spectrum. We investigated three phenomenologically viable parameter scenarios in more detail, where one of these particles is identified with the $125 \mathrm{GeV}$ Higgs boson. We considered parameter sets of this model where this boson has SM-like couplings to the quarks, leptons, and weak gauge bosons. The masses of the other two neutral Higgs bosons were assumed to lie above the $t \bar{t}$ threshold, with top-quark Yukawa couplings that are slightly larger than the SM top-quark Yukawa coupling.

For these three parameter sets we investigated heavy Higgs boson production and decay to $t \bar{t}$ at the LHC operating at $13 \mathrm{TeV}$. We computed the inclusive $t \bar{t}$ cross section and the $t \bar{t}$ invariant mass distribution with and without the two heavy Higgs resonances. Assuming an integrated luminosity of $100 \mathrm{fb}^{-1}$, we estimated, for appropriately chosen $M_{t \bar{t}}$ intervals and our three parameter scenarios, the significances for detecting a heavy Higgs signal in the $t \bar{t}$ dileptonic and lepton plus jets decay channels. In addition we computed the top-quark transverse momentum distribution and, for selected $M_{t \bar{t}}$ windows, the top-quark rapidity distribution and that of the cosine of the Collins-Soper angle. We showed also that the so-far negative results by the CMS and ATLAS experiment on searches for heavy spin-zero resonances in the $t \bar{t}$ channel at the LHC (8 TeV) do not exclude our parameter scenarios.

Because the masses of the heavy Higgs resonances are not known, we propose to scan the $t \bar{t}$ invariant mass distribution with a sliding $M_{t \bar{t}}$ window of width $\Delta M_{t \bar{t}} \sim 80 \mathrm{GeV}$. Mass bins of this size are experimentally feasible, at least for $M_{t \bar{t}} \lesssim 1 \mathrm{TeV}$. Our analysis of Sec. 5 shows that, for our parameter scenarios, the significances for detecting a heavy Higgs resonance exceed $3 \sigma$ only if the uncertainties involved in measuring $M_{t \bar{t}}$ windows and modeling the nonresonant $t \bar{t}$ background can be pushed down to a level of $\sim 1 \%$. Such a precision is unrealistic at present, but since both theoretical and experimental uncertainties in measuring the (binned) 
$t \bar{t}$ cross section have been significantly reduced in recent years, one may be optimistic and expect further improvements also in the future. Full multivariate experimental analyses, which increase the sensitivity, would also use information from other distributions, including those considered in Sec. 5. A further set of observables, which we plan to investigate in future work, includes $t \bar{t}$ spin correlation and $t, \bar{t}$ polarization observables. Such observables show, in selected $M_{t \bar{t}}$ windows, also some sensitivity to heavy Higgs resonances and potentially allow one to discriminate between a scalar, a pseudoscalar, and a $C P$ mixture.

\section{Acknowledgments}

W.B. thanks A. Brandenburg for a discussion. This work was supported in part by Deutsche Forschungsgemeinschaft (DFG) Grant No. SFB-TR09. P. G. was supported by DFG through Graduiertenkolleg Grant No. GRK 1504 and C. M. through DFG Graduiertenkolleg Grant No. GRK 1675. The work of Z.G. S. was supported by National Natural Science Foundation of China and by Natural Science Foundation of Shandong Province.

\section{A. Description of Higgs resonances}

The unstable particles which are involved in the reactions considered in this paper are the weak gauge bosons, and the three neutral Higgs bosons of the 2HDM extension of the SM. The charged Higgs boson does not play any role here-see the 2HDM scenarios considered in Sec. 3. The top quark, too, is of course unstable, but in this paper we consider reactions at the level of $t$ and $\bar{t}$ final states. The following discussion applies also if top-quark decays are incorporated into the analysis. While we take higher order QCD corrections to the parton reactions $a b \rightarrow t \bar{t}+X$ into account we emphasize that we incorporate the weak gauge boson and Yukawa interactions only to leading order. This is important to keep in mind in the following discussion.

As argued above, the top quark can be treated in the narrow width approximation. The $Z$ boson appears in $t \bar{t}+X$ production as $s$-channel resonance only far off shell, $Z^{*} \rightarrow t \bar{t}+X$. Hence its width can be safely neglected. The same statement applies to the light Higgs boson $\phi_{1}$ with ${ }^{6}$ $m_{1}=125 \mathrm{GeV}$. The $W^{ \pm}$boson is resonantly produced in $t$ and $\bar{t}$ decay, respectively. As we work to lowest order in the weak gauge couplings this can be incorporated by using the width $\Gamma_{W}$ in the $W$-boson propagator.

In the resonant production of the heavy Higgs bosons, $a b \rightarrow \phi_{2,3} \rightarrow t \bar{t}+X$, the finite width effects must be accounted for. The adequate method which respects gauge invariance also with respect to higher order electroweak corrections is the so-called complex mass scheme [55-57]. Let us illustrate this method for the case at hand, using only one $\phi$. We consider the amplitude for the process $a b \rightarrow \phi \rightarrow t \bar{t}+X$, including only factorizable QCD corrections. The

\footnotetext{
${ }^{6}$ The total width of $\phi_{1}$ is SM-like, i.e. very small in the $2 \mathrm{HDM}$ scenarios considered below, $\Gamma_{1} \sim 4 \mathrm{MeV}$.
} 
nonfactorizable QCD corrections are analyzed in Sec. 4.2 and Appendix B. In the complex mass scheme the amplitude is of the form

$$
\mathcal{M}_{i j \rightarrow f}=S_{i j}\left(k^{2}, \cdots\right) i P\left(k^{2}\right) S_{f}\left(k^{2}, \cdots\right)+\mathcal{N}, \quad P\left(k^{2}\right)=\frac{1}{k^{2}-\mu_{\phi}^{2}},
$$

where the complex mass parameter $\mu_{\phi}^{2}$ is the pole of the full $\phi$ propagator, i.e., the point in the complex $s$ plane where the renormalized Higgs boson self energy $\Pi(s)$ vanishes:

$$
\Pi\left(\mu_{\phi}^{2}\right)=0 .
$$

The symbol $\mathcal{N}$ in Eq. (76) denotes the nonresonant contribution to the scattering matrix element. The complex mass parameter must be used wherever the $\phi$ mass squared occurs in the scattering amplitude. However, as we compute (76) only to lowest order in the weak and Yukawa interactions, $\mu_{\phi}^{2}$ does not appear in the loops contributing to $S_{i j}$ and $S_{f}$, but only in $P(s)$. We parametrize

$$
\mu_{\phi}^{2}=m^{2}-i m \Gamma \text {. }
$$

The meaning of $m$ and $\Gamma$ is as follows. The relation between the renormalized and bare Higgs boson self energy is, to one-loop order in the electroweak and Yukawa couplings,

$$
\Pi\left(k^{2}\right)=\Pi_{0}\left(k^{2}\right)-\delta \mu_{\phi}^{2}+\left(k^{2}-\mu_{\phi}^{2}\right) \delta \mathcal{Z}_{\phi},
$$

where $\mathcal{Z}_{\phi}=1+\delta \mathcal{Z}_{\phi}$ is the complex Higgs wave-function renormalization constant and $\delta \mu_{\phi}^{2}$ is the complex mass counterterm. The relation between the real bare Higgs mass squared, $m_{0}^{2}$, and the complex renormalized mass squared $\mu_{\phi}^{2}$ is

$$
m_{0}^{2}=\mu_{\phi}^{2}+\delta \mu_{\phi}^{2}
$$

The condition (77) is the complex-mass-scheme generalization of the usual on-shell renormalization condition. Using (79) it implies that

$$
\delta \mu_{\phi}^{2}=\Pi_{0}\left(\mu_{\phi}^{2}\right) .
$$

Now we use the parametrization (78) and the relation $(80)$, i.e., $\delta \mu_{\phi}^{2}=m_{0}^{2}-\mu_{\phi}^{2}$. Taking the real part of this relation shows that $m$ can be interpreted as the on-shell mass of $\phi$. Taking the imaginary part gives the equation

$$
m \Gamma=\operatorname{Im} \prod_{0}\left(m^{2}-i m \Gamma\right),
$$

which in general can be solved iteratively for $\Gamma$. Expanding this relation around $m$, one gets to one-loop order in the electroweak and Yukawa couplings [56]:

$$
m \Gamma=\operatorname{Im} \Pi_{0}\left(m^{2}\right)-m \Gamma \operatorname{Re} \Pi_{0}^{\prime}\left(m^{2}\right),
$$

where the prime denotes the derivative with respect to $k^{2}$. Again, this equation may be solved iteratively for $\Gamma$. Because we work to lowest order in the weak and Yukawa couplings we can neglect the second term in 83 . Then $\Gamma$ is the total $\phi$-boson width in the usual on-shell scheme.

In summary we use (76) where $\mu_{\phi}^{2}$ is determined by the on-shell mass and total width of the respective Higgs boson $\phi_{2}, \phi_{3}$. 


\section{B. Cancellation of real and virtual nonfactorizable corrections in the soft gluon approximation}

As an example for the cancellation of real and virtual nonfactorizable corrections in the SGA we take the amplitude depicted in Fig. 6. The dotted red cut in Fig. 6 corresponds to the interference of two Feynman diagrams contributing to the real corrections and the dashed green cut indicates the interference of the one-loop diagram shown in Fig. 5] with the $s$-channel QCD Born diagram. We start with the latter interference. In the SGA the loop integral for the amplitude shown in Fig. 5 reduces to the scalar integral

$$
\int \frac{d^{4} \ell}{(2 \pi)^{4}} \frac{\mathcal{N}_{\text {virt }}}{\left[\ell^{2}+i \varepsilon\right]\left[-2 \ell \cdot p_{1}+i \varepsilon\right]\left[-2 \ell \cdot\left(p_{1}+p_{2}\right)+s-m_{\phi}^{2}+i \varepsilon\right]\left[-2 \ell \cdot k_{1}+i \varepsilon\right]},
$$

where $\ell$ is the loop momentum which is required to be soft within the SGA. The numerator $\mathcal{N}_{\text {virt }}$ in the SGA is obtained from the exact numerator by setting $\ell=0$. The momenta of the incoming gluons are denoted $p_{1}$ and $p_{2}$, and $k_{1}$ is the momentum of the outgoing top quark. In the last three square brackets of the denominator of (84) the $\ell^{2}$ terms are neglected, which is also part of the SGA. The integration over the $\ell^{0}$ component is performed in the complex $\ell^{0}$ plane using the residue theorem. Closing the contour in the lower half of the plane the residue of the pole of the first propagator in Eq. (84) is picked up. The location of the poles of the other propagators is in the upper half of the $\ell^{0}$ plane. Ignoring the numerator the following result is obtained:

$$
\begin{aligned}
& -i \int \frac{d^{3} \ell}{(2 \pi)^{3}} \frac{1}{2|\boldsymbol{\ell}|} \frac{1}{\left[-2|\boldsymbol{\ell}| p_{1}^{0}+2 \boldsymbol{\ell} \cdot \mathbf{p}_{1}+i \varepsilon\right]} \\
& \times \frac{1}{\left[-2|\boldsymbol{\ell}|\left(p_{1}^{0}+p_{2}^{0}\right)+2 \boldsymbol{\ell}\left(\mathbf{p}_{1}+\mathbf{p}_{2}\right)+s-m_{\phi}^{2}+i \varepsilon\right]\left[-2|\boldsymbol{\ell}| k_{1}^{0}+2 \boldsymbol{\ell} \cdot \mathbf{k}_{1}+i \varepsilon\right]} \\
& =i \int \frac{d^{3} \ell}{(2 \pi)^{3}} \frac{1}{2 \ell 0} \frac{1}{\left[-2 \ell \cdot p_{1}+i \varepsilon\right]\left[-2 \ell \cdot\left(p_{1}+p_{2}\right)+s-m_{\phi}^{2}+i \varepsilon\right]\left[2 \ell \cdot k_{1}-i \varepsilon\right]}
\end{aligned}
$$

with $|\boldsymbol{\ell}|=\ell^{0}$. The integration measure $d^{3} \ell /\left[(2 \pi)^{3} 2 \ell^{0}\right]$ is identical to the Lorentz invariant phase-space measure of the gluon. What remains to be done is to show that Eq. (85) including the numerator indeed corresponds to the phase-space integral over the soft gluon in the corresponding real correction contribution. Thus we analyze the interference term indicated in Fig. 6 by the dotted red cut. After applying the SGA this interference leads to the following propagator structure,

$$
\frac{1}{\left[-2 q \cdot p_{1}+i \varepsilon\right]\left[-2 q \cdot\left(p_{1}+p_{2}\right)+s-m_{\phi}^{2}+i \varepsilon\right]\left[2 q \cdot k_{1}-i \varepsilon\right]},
$$

where $q$ is the four-momentum of the (soft) gluon. Taking the phase-space integration over the soft gluon momentum $q$ into account,

$$
\int \frac{d^{3} q}{(2 \pi)^{3}} \frac{1}{2 q^{0}} \frac{1}{\left[-2 q \cdot p_{1}+i \varepsilon\right]\left[-2 q \cdot\left(p_{1}+p_{2}\right)+s-m_{\phi}^{2}+i \varepsilon\right]\left[2 q \cdot k_{1}-i \varepsilon\right]},
$$


the two expressions Eq. (85) and Eq. (87) are identical up to a factor of $i$.

So far only the denominators were investigated and the numerators of the amplitudes were ignored. It remains to show that they are identical up to a sign. The real radiation amplitude leads to

$$
\begin{aligned}
\mathcal{N}_{\text {real }} & =\operatorname{Tr}\left[\left(k_{1}+m_{t}\right) V_{\phi t t}\left(k_{2}-m_{t}\right) V_{g t t, \alpha_{2}}^{c_{2}}\left(\not k_{1}+m_{t}\right) V_{g t t}^{\rho^{\prime}, c_{3}}\right] \\
& \times V_{g g g}^{\mu^{\prime} \nu^{\prime} \alpha_{2}, a b c_{2}}\left(p_{1}, p_{2},-p_{1}-p_{2}\right) V_{g g g}^{\mu \alpha_{1} \rho, a c_{1} c_{3}}\left(p_{1},-p_{1}, 0\right) V_{g g \phi, \alpha_{1}}^{v, b c_{1}}\left(p_{2}, p_{1}\right) \\
& \times \frac{\mathcal{P}_{\mu \mu^{\prime}}\left(p_{1}, p_{2}\right) \mathcal{P}_{v \nu^{\prime}}\left(p_{2}, p_{1}\right) \mathcal{P}_{\rho \rho^{\prime}}(q, n)}{\left(p_{1}+p_{2}\right)^{2}}
\end{aligned}
$$

where

$$
\begin{aligned}
V_{\phi t t} & =-i \frac{m_{t}}{v}\left(a_{t}-i b_{t} \gamma_{5}\right), \\
V_{g t t}^{\mu, a} & =-i g_{s} t^{a} \gamma^{\mu}, \\
V_{g g g}^{\mu \nu \rho, a b c}\left(p_{1}, p_{2}, p_{3}\right) & =-g_{s} f^{a b c}\left(g^{\mu \nu}\left(p_{1}-p_{2}\right)^{\rho}+g^{\nu \rho}\left(p_{2}-p_{3}\right)^{\mu}+g^{\rho \mu}\left(p_{3}-p_{1}\right)^{v}\right), \\
V_{g g \phi}^{\mu \nu, a b}\left(p_{1}, p_{2}\right) & =-4 i \delta^{a b}\left[f_{S}^{(0)}\left(g^{\mu v} p_{1} \cdot p_{2}-p_{1}^{v} p_{2}^{\mu}\right)-2 f_{P}^{(0)} \epsilon^{\mu \nu \rho \sigma} p_{1 \rho} p_{2 \sigma}\right],
\end{aligned}
$$

and $\mathcal{P}_{\alpha \alpha^{\prime}}$ is the gluon polarization sum

$$
\mathcal{P}_{\alpha \alpha^{\prime}}(p, n)=-g_{\alpha \alpha^{\prime}}+\frac{p_{\alpha} n_{\alpha^{\prime}}+p_{\alpha^{\prime}} n_{\alpha}}{p \cdot n}, \quad n^{2}=0,
$$

where $n$ is an arbitrary lightlike vector. Instead of the polarization sum in Eq. (93) we use

$$
\mathcal{P}_{\rho \rho^{\prime}}(q, n)=-g_{\rho \rho^{\prime}},
$$

because in the present case there are no diagrams where the external soft gluon could be replaced by a ghost. With this relation we obtain:

$$
\begin{aligned}
\mathcal{N}_{\text {real }} & =-\operatorname{Tr}\left[\left(k_{1}+m_{t}\right) V_{\phi t t}\left(k_{2}-m_{t}\right) V_{g t t, \alpha_{2}}^{c_{2}}\left(\not k_{1}+m_{t}\right) V_{g t t}^{\alpha_{3}, c_{3}}\right] \\
& \times V_{g g g}^{\mu^{\prime} v^{\prime} \alpha_{2}, a b c_{2}}\left(p_{1}, p_{2},-p_{1}-p_{2}\right) V_{g g g, \alpha_{3}}^{\mu \alpha \alpha_{1} a c_{1} c_{3}}\left(p_{1},-p_{1}, 0\right) V_{g g \phi, \alpha_{1}}^{v, b c_{1}}\left(p_{2}, p_{1}\right) \\
& \times \frac{\mathcal{P}_{\mu \mu^{\prime}}\left(p_{1}, p_{2}\right) \mathcal{P}_{v v^{\prime}}\left(p_{2}, p_{1}\right)}{\left(p_{1}+p_{2}\right)^{2}} .
\end{aligned}
$$

The numerator of $\mathcal{N}_{\text {virt }}$ of the virtual correction contribution 84 is computed in straightforward fashion in the SGA. One obtains

$$
\mathcal{N}_{\text {virt }}=i \mathcal{N}_{\text {real }} \text {. }
$$

Therefore

$$
\begin{aligned}
& i \int \frac{d^{3} \ell}{(2 \pi)^{3}} \frac{1}{2 \ell^{0}} \frac{\mathcal{N}_{\text {virt }}}{\left[-2 \ell \cdot p_{1}+i \varepsilon\right]\left[-2 \ell \cdot\left(p_{1}+p_{2}\right)+s-m_{\phi}^{2}+i \varepsilon\right]\left[2 \ell \cdot k_{1}-i \varepsilon\right]} \\
& =-\int \frac{d^{3} \ell}{(2 \pi)^{3}} \frac{1}{2 \ell^{0}} \frac{\mathcal{N}_{\text {real }}}{\left[-2 \ell \cdot p_{1}+i \varepsilon\right]\left[-2 \ell \cdot\left(p_{1}+p_{2}\right)+s-m_{\phi}^{2}+i \varepsilon\right]\left[2 \ell \cdot k_{1}-i \varepsilon\right]}
\end{aligned}
$$


which exactly cancels the contribution from the real correction,

$$
\int \frac{d^{3} q}{(2 \pi)^{3}} \frac{1}{2 q^{0}} \frac{\mathcal{N}_{\text {real }}}{\left[-2 q \cdot p_{1}+i \varepsilon\right]\left[-2 q \cdot\left(p_{1}+p_{2}\right)+s-m_{\phi}^{2}+i \varepsilon\right]\left[2 q \cdot k_{1}-i \varepsilon\right]} .
$$

Note that

$$
\delta^{(4)}\left(p_{1}+p_{2}-k_{1}-k_{2}-q\right) \stackrel{\mathrm{SGA}}{\longrightarrow} \delta^{(4)}\left(p_{1}+p_{2}-k_{1}-k_{2}\right)
$$

in the phase-space integration of (98). Thus the four-momentum conservation matches the one in the phase-space integration of (97).

\section{References}

[1] ATLAS Collaboration, G. Aad et al., Observation of a new particle in the search for the Standard Model Higgs boson with the ATLAS detector at the LHC, Phys. Lett. B716 (2012) 1-29, arXiv: 1207.7214 [hep-ex].

[2] CMS Collaboration, S. Chatrchyan et al., Observation of a new boson at a mass of 125 GeV with the CMS experiment at the LHC, Phys. Lett. B716 (2012) 30-61, arXiv: 1207.7235 [hep-ex].

[3] CMS Collaboration, V. Khachatryan et al., Precise determination of the mass of the Higgs boson and tests of compatibility of its couplings with the standard model predictions using proton collisions at 7 and 8 TeV, Eur. Phys. J. C75 no. 5, (2015) 212, arXiv: 1412.8662 [hep-ex].

[4] ATLAS Collaboration, G. Aad et al., Measurements of the Higgs boson production and decay rates and coupling strengths using pp collision data at $\sqrt{s}=7$ and $8 \mathrm{TeV}$ in the ATLAS experiment, Eur. Phys. J. C76 no. 1, (2016) 6, arXiv: 1507.04548 [hep-ex].

[5] A. Djouadi, The Anatomy of electro-weak symmetry breaking. II. The Higgs bosons in the minimal supersymmetric model, Phys. Rept. 459 (2008) 1-241, arXiv:hep-ph/0503173 [hep-ph]

[6] G. C. Branco, P. M. Ferreira, L. Lavoura, M. N. Rebelo, M. Sher, and J. P. Silva, Theory and phenomenology of two-Higgs-doublet models, Phys. Rept. 516 (2012) 1-102, arXiv:1106.0034 [hep-ph].

[7] ATLAS Collaboration, Measurements of the properties of the Higgs-like boson in the four lepton decay channel with the ATLAS detector using $25 \mathrm{fb}^{-1}$ of proton-proton collision data, ATLAS-CONF-2013-013,

[8] CMS Collaboration, V. Khachatryan et al., Search for a pseudoscalar boson decaying into $a \mathrm{Z}$ boson and the $125 \mathrm{GeV}$ Higgs boson in $\ell^{+} \ell^{-} b \bar{b}$ final states, Phys. Lett. B748 (2015) 221-243, arXiv: 1504.04710 [hep-ex]. 
[9] ATLAS Collaboration, G. Aad et al., Search For Higgs Boson Pair Production in the $\gamma \gamma b \bar{b}$ Final State using pp Collision Data at $\sqrt{s}=8 \mathrm{TeV}$ from the ATLAS Detector, Phys. Rev. Lett. 114 no. 8, (2015) 081802, arXiv: 1406.5053 [hep-ex].

[10] ATLAS Collaboration, G. Aad et al., Search for a CP-odd Higgs boson decaying to Zh in pp collisions at $\sqrt{s}=8 T e V$ with the ATLAS detector, Phys. Lett. B744 (2015) 163-183, arXiv: 1502.04478 [hep-ex].

[11] CMS Collaboration, V. Khachatryan et al., Search for a Higgs Boson in the Mass Range from 145 to $1000 \mathrm{GeV}$ Decaying to a Pair of W or Z Bosons, JHEP 10 (2015) 144. arXiv: 1504.00936 [hep-ex].

[12] ATLAS, CMS Collaboration, Y. Nagai, Higgs Search in b $\bar{b}$ Signatures at ATLAS and CMS, PoS Beauty2013 (2013) 001, arXiv: 1306.1784 [hep-ex].

[13] CMS Collaboration, V. Khachatryan et al., Search for resonant pair production of Higgs bosons decaying to two bottom quarkantiquark pairs in protonproton collisions at 8 $T e V$, Phys. Lett. B749 (2015) 560-582, arXiv: 1503.04114 [hep-ex]

[14] CMS Collaboration, V. Khachatryan et al., Search for neutral MSSM Higgs bosons decaying to a pair of tau leptons in pp collisions, JHEP 10 (2014) 160. arXiv: 1408.3316 [hep-ex].

[15] ATLAS Collaboration, G. Aad et al., Search for neutral Higgs bosons of the minimal supersymmetric standard model in pp collisions at $\sqrt{s}=8 \mathrm{TeV}$ with the ATLAS detector, JHEP 11 (2014) 056, arXiv: 1409.6064 [hep-ex].

[16] K. J. F. Gaemers and F. Hoogeveen, Higgs Production and Decay Into Heavy Flavors With the Gluon Fusion Mechanism, Phys. Lett. B146 (1984) 347.

[17] D. Dicus, A. Stange, and S. Willenbrock, Higgs decay to top quarks at hadron colliders, Phys. Lett. B333 (1994) 126-131, arXiv: hep-ph/9404359 [hep-ph].

[18] W. Bernreuther and A. Brandenburg, Tracing CP violation in the production of top quark pairs by multiple TeV proton proton collisions, Phys. Rev. D49 (1994) 4481-4492, arXiv:hep-ph/9312210 [hep-ph]

[19] W. Bernreuther, M. Flesch, and P. Haberl, Signatures of Higgs bosons in the top quark decay channel at hadron colliders, Phys. Rev. D58 (1998) 114031. arXiv:hep-ph/9709284 [hep-ph].

[20] W. Bernreuther, A. Brandenburg, and M. Flesch, Effects of Higgs sector CP violation in top quark pair production at the LHC, arXiv:hep-ph/9812387 [hep-ph].

[21] V. Barger, T. Han, and D. G. E. Walker, Top Quark Pairs at High Invariant Mass: A Model-Independent Discriminator of New Physics at the LHC, Phys. Rev. Lett. 100 (2008) 031801, arXiv:hep-ph/0612016 [hep-ph].

[22] R. Frederix and F. Maltoni, Top pair invariant mass distribution: A Window on new 
physics, JHEP 01 (2009) 047, arXiv:0712.2355 [hep-ph].

[23] V. Barger, W.-Y. Keung, and B. Yencho, Azimuthal Correlations in Top Pair Decays and The Effects of New Heavy Scalars, Phys. Rev. D85 (2012) 034016, arXiv: 1112.5173 [hep-ph].

[24] N. Craig, F. D'Eramo, P. Draper, S. Thomas, and H. Zhang, The Hunt for the Rest of the Higgs Bosons, JHEP 06 (2015) 137, arXiv: 1504 . 04630 [hep-ph].

[25] S. Jung, J. Song, and Y. W. Yoon, Dip or nothingness of a Higgs resonance from the interference with a complex phase, Phys. Rev. D92 no. 5, (2015) 055009. arXiv: 1505.00291 [hep-ph].

[26] B. Bhattacherjee, A. Chakraborty, and A. Choudhury, Status of the MSSM Higgs sector using global analysis and direct search bounds, and future prospects at the High Luminosity LHC, Phys. Rev. D92 no. 9, (2015) 093007, arXiv: 1504.04308 [hep-ph].

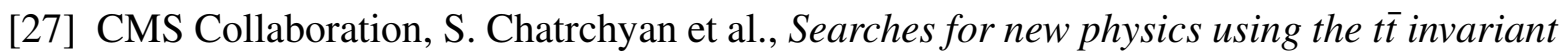
mass distribution in pp collisions at $\sqrt{s}=8 T e V$, Phys. Rev. Lett. 111 no. 21, (2013) 211804, arXiv:1309.2030 [hep-ex]. [Erratum: Phys. Rev. Lett.112,119903(2014)].

[28] ATLAS Collaboration, G. Aad et al., A search for $t \bar{t}$ resonances using lepton-plus-jets events in proton-proton collisions at $\sqrt{s}=8 \mathrm{TeV}$ with the ATLAS detector, JHEP 08 (2015) 148, arXiv: 1505.07018 [hep-ex].

[29] M. Kramer, E. Laenen, and M. Spira, Soft gluon radiation in Higgs boson production at the LHC, Nucl. Phys. B511 (1998) 523-549, arXiv: hep-ph/9611272 [hep-ph].

[30] J. C. Collins and D. E. Soper, Angular Distribution of Dileptons in High-Energy Hadron Collisions, Phys. Rev. D16 (1977) 2219.

[31] A. W. El Kaffas, W. Khater, O. M. Ogreid, and P. Osland, Consistency of the two Higgs doublet model and CP violation in top production at the LHC, Nucl. Phys. B775 (2007) 45-77, arXiv:hep-ph/0605142 [hep-ph].

[32] B. Grzadkowski, O. M. Ogreid, and P. Osland, Measuring CP violation in Two-Higgs-Doublet models in light of the LHC Higgs data, JHEP 11 (2014) 084, arXiv: 1409.7265 [hep-ph].

[33] W. Bernreuther, T. Schroder, and T. N. Pham, CP violating dipole form-factors in $e^{+} e^{-} \rightarrow t \bar{t}$, Phys. Lett. B279 (1992) 389-396.

[34] S. Inoue, M. J. Ramsey-Musolf, and Y. Zhang, CP-violating phenomenology of flavor conserving two Higgs doublet models, Phys. Rev. D89 no. 11, (2014) 115023, arXiv: 1403.4257 [hep-ph].

[35] C.-Y. Chen, S. Dawson, and Y. Zhang, Complementarity of LHC and EDMs for Exploring Higgs CP Violation, JHEP 06 (2015) 056, arXiv: 1503.01114 [hep-ph]. 
[36] C. Mellein, Doctoral Thesis, RWTH Aachen University (2015), unpublished,.

[37] F. Mahmoudi and O. Stal, Flavor constraints on the two-Higgs-doublet model with general Yukawa couplings, Phys. Rev. D81 (2010) 035016, arXiv: 0907. 1791 [hep-ph].

[38] T. Hermann, M. Misiak, and M. Steinhauser, $\bar{B} \rightarrow X_{s} \gamma$ in the Two Higgs Doublet Model up to Next-to-Next-to-Leading Order in QCD, JHEP 11 (2012) 036, arXiv: 1208.2788 [hep-ph].

[39] O. Eberhardt, U. Nierste, and M. Wiebusch, Status of the two-Higgs-doublet model of type II, JHEP 07 (2013) 118, arXiv: 1305.1649 [hep-ph].

[40] A. Djouadi, J. Kalinowski, and M. Spira, HDECAY: A Program for Higgs boson decays in the standard model and its supersymmetric extension, Comput. Phys. Commun. 108 (1998) 56-74, arXiv: hep-ph/9704448 [hep-ph]

[41] D. Eriksson, J. Rathsman, and O. Stal, 2HDMC: Two-Higgs-Doublet Model Calculator Physics and Manual, Comput. Phys. Commun. 181 (2010) 189-205. arXiv:0902.0851 [hep-ph]

[42] E. Braaten and J. P. Leveille, Higgs Boson Decay and the Running Mass, Phys. Rev. D22 (1980) 715 .

[43] M. Drees and K.-i. Hikasa, NOTE ON QCD CORRECTIONS TO HADRONIC HIGGS DECAY, Phys. Lett. B240 (1990) 455. [Erratum: Phys. Lett.B262,497(1991)].

[44] M. Spira, A. Djouadi, D. Graudenz, and P. M. Zerwas, Higgs boson production at the LHC, Nucl. Phys. B453 (1995) 17-82, arXiv: hep-ph/9504378 [hep-ph].

[45] A. Dabelstein and W. Hollik, Electroweak corrections to the fermionic decay width of the standard Higgs boson, Z. Phys. C53 (1992) 507-516.

[46] J. Fleischer and F. Jegerlehner, Radiative Corrections to Higgs Decays in the Extended Weinberg-Salam Model, Phys. Rev. D23 (1981) 2001-2026.

[47] W. Grimus, L. Lavoura, O. M. Ogreid, and P. Osland, A Precision constraint on multi-Higgs-doublet models, J. Phys. G35 (2008) 075001, arXiv: 0711.4022 [hep-ph].

[48] W. Grimus, L. Lavoura, O. M. Ogreid, and P. Osland, The Oblique parameters in multi-Higgs-doublet models, Nucl. Phys. B801 (2008) 81-96, arXiv:0802 . 4353 [hep-ph].

[49] H. E. Haber and D. O'Neil, Basis-independent methods for the two-Higgs-doublet model III: The CP-conserving limit, custodial symmetry, and the oblique parameters $S$, $T, U$, Phys. Rev. D83 (2011) 055017, arXiv: 1011.6188 [hep-ph].

[50] C. A. Baker et al., An Improved experimental limit on the electric dipole moment of the neutron, Phys. Rev. Lett. 97 (2006) 131801, arXiv:hep-ex/0602020 [hep-ex]. 
[51] ACME Collaboration, J. Baron et al., Order of Magnitude Smaller Limit on the Electric Dipole Moment of the Electron, Science 343 (2014) 269-272, arXiv: 1310. 7534 [physics.atom-ph].

[52] J. H. Kuhn, A. Scharf, and P. Uwer, Weak Interactions in Top-Quark Pair Production at Hadron Colliders: An Update, Phys. Rev. D91 no. 1, (2015) 014020, arXiv: 1305.5773 [hep-ph].

[53] P. Nason, S. Dawson, and R. K. Ellis, The Total Cross-Section for the Production of Heavy Quarks in Hadronic Collisions, Nucl. Phys. B303 (1988) 607.

[54] W. Beenakker, H. Kuijf, W. L. van Neerven, and J. Smith, QCD Corrections to Heavy Quark Production in p anti-p Collisions, Phys. Rev. D40 (1989) 54-82.

[55] A. Denner, S. Dittmaier, M. Roth, and D. Wackeroth, Predictions for all processes $e^{+} e^{-} \rightarrow 4$ fermions $+\gamma$, Nucl. Phys. B560 (1999) 33-65, arXiv: hep-ph/9904472 [hep-ph].

[56] A. Denner, S. Dittmaier, M. Roth, and L. H. Wieders, Electroweak corrections to charged-current $e^{+} e^{-} \rightarrow 4$ fermion processes: Technical details and further results, Nucl. Phys. B724 (2005) 247-294, arXiv: hep-ph/0505042 [hep-ph]. [Erratum: Nucl. Phys.B854,504(2012)].

[57] M. Nowakowski and A. Pilaftsis, On gauge invariance of Breit-Wigner propagators, Z. Phys. C60 (1993) 121-126, arXiv:hep-ph/9305321 [hep-ph].

[58] R. Harlander and P. Kant, Higgs production and decay: Analytic results at next-to-leading order QCD, JHEP 12 (2005) 015, arXiv: hep-ph/0509189 [hep-ph].

[59] A. Djouadi, M. Spira, and P. M. Zerwas, Production of Higgs bosons in proton colliders: QCD corrections, Phys. Lett. B264 (1991) 440-446.

[60] R. V. Harlander and W. B. Kilgore, Soft and virtual corrections to proton proton to $H+$ $x$ at NNLO, Phys. Rev. D64 (2001) 013015, arXiv:hep-ph/0102241 [hep-ph].

[61] K. G. Chetyrkin, B. A. Kniehl, M. Steinhauser, and W. A. Bardeen, Effective QCD interactions of CP odd Higgs bosons at three loops, Nucl. Phys. B535 (1998) 3-18, arXiv:hep-ph/9807241 [hep-ph].

[62] R. V. Harlander and W. B. Kilgore, Production of a pseudoscalar Higgs boson at hadron colliders at next-to-next-to leading order, JHEP 10 (2002) 017. arXiv:hep-ph/0208096 [hep-ph].

[63] V. S. Fadin, V. A. Khoze, and A. D. Martin, Interference radiative phenomena in the production of heavy unstable particles, Phys. Rev. D49 (1994) 2247-2256.

[64] K. Melnikov and O. I. Yakovlev, Final state interaction in the production of heavy unstable particles, Nucl. Phys. B471 (1996) 90-120, arXiv: hep-ph/9501358 


\section{[hep-ph].}

[65] W. Beenakker, A. P. Chapovsky, and F. A. Berends, Nonfactorizable corrections to $W$ pair production: Methods and analytic results, Nucl. Phys. B508 (1997) 17-63. arXiv:hep-ph/9707326 [hep-ph]

[66] S. Dittmaier, A. Huss, and C. Schwinn, Mixed QCD-electroweak $O\left(\alpha_{s} \alpha\right)$ corrections to Drell-Yan processes in the resonance region: pole approximation and non-factorizable corrections, Nucl. Phys. B885 (2014) 318-372, arXiv: 1403.3216 [hep-ph].

[67] P. Nason, S. Dawson, and R. K. Ellis, The One Particle Inclusive Differential Cross-Section for Heavy Quark Production in Hadronic Collisions, Nucl. Phys. B327 (1989) 49-92. [Erratum: Nucl. Phys.B335,260(1990)].

[68] W. Beenakker, W. L. van Neerven, R. Meng, G. A. Schuler, and J. Smith, $Q C D$ corrections to heavy quark production in hadron hadron collisions, Nucl. Phys. B351 (1991) 507-560.

[69] W. Bernreuther, A. Brandenburg, Z. G. Si, and P. Uwer, Top quark spin correlations at hadron colliders: Predictions at next-to-leading order QCD, Phys. Rev. Lett. 87 (2001) 242002, arXiv:hep-ph/0107086 [hep-ph].

[70] W. Bernreuther, A. Brandenburg, Z. G. Si, and P. Uwer, Top quark pair production and decay at hadron colliders, Nucl. Phys. B690 (2004) 81-137, arXiv: hep-ph/0403035 [hep-ph].

[71] G. Passarino and M. J. G. Veltman, One Loop Corrections for $e^{+} e^{-}$Annihilation Into $\mu^{+} \mu^{-}$in the Weinberg Model, Nucl. Phys. B160 (1979) 151.

[72] W. Bernreuther, R. Bonciani, T. Gehrmann, R. Heinesch, P. Mastrolia, and E. Remiddi, Decays of scalar and pseudoscalar Higgs bosons into fermions: Two-loop QCD corrections to the Higgs-quark-antiquark amplitude, Phys. Rev. D72 (2005) 096002, arXiv:hep-ph/0508254 [hep-ph]

[73] S. Catani and M. H. Seymour, A General algorithm for calculating jet cross-sections in NLO QCD, Nucl. Phys. B485 (1997) 291-419, arXiv: hep-ph/9605323 [hep-ph]. [Erratum: Nucl. Phys.B510,503(1998)].

[74] S. Catani, S. Dittmaier, M. H. Seymour, and Z. Trocsanyi, The Dipole formalism for next-to-leading order QCD calculations with massive partons, Nucl. Phys. B627 (2002) 189-265, arXiv:hep-ph/0201036 [hep-ph].

[75] W. Bernreuther and Z.-G. Si, Distributions and correlations for top quark pair production and decay at the Tevatron and LHC., Nucl. Phys. B837 (2010) 90-121, arXiv:1003.3926 [hep-ph].

[76] H.-L. Lai, M. Guzzi, J. Huston, Z. Li, P. M. Nadolsky, J. Pumplin, and C. P. Yuan, New parton distributions for collider physics, Phys. Rev. D82 (2010) 074024, arXiv:1007.2241 [hep-ph]. 
[77] LHC Higgs Cross Section Working Group Collaboration, S. Dittmaier et al., Handbook of LHC Higgs Cross Sections: 1. Inclusive Observables, arXiv: 1101.0593 [hep-ph].

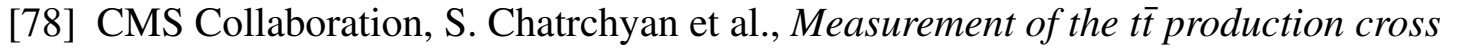
section in the dilepton channel in pp collisions at $\sqrt{s}=8 \mathrm{TeV}$, JHEP 02 (2014) 024, arXiv: 1312.7582 [hep-ex], [Erratum: JHEP02,102(2014)].

[79] CMS Collaboration, V. Khachatryan et al., Measurement of the differential cross section for top quark pair production in pp collisions at $\sqrt{s}=8 \mathrm{TeV}$, Eur. Phys. J. C75 no. 11, (2015) 542, arXiv: 1505.04480 [hep-ex].

[80] T. P. Li and Y. Q. Ma, Analysis methods for results in gamma-ray astronomy, Astrophys. J. 272 (1983) 317-324.

[81] R. D. Cousins, J. T. Linnemann, and J. Tucker, Evaluation of three methods for calculating statistical significance when incorporating a systematic uncertainty into a test of the background-only hypothesis for a Poisson process, Nucl. Instrum. Meth. A595 (2008) 480-501.

[82] M. Czakon, P. Fiedler, and A. Mitov, Total Top-Quark Pair-Production Cross Section at Hadron Colliders Through $O\left(\alpha_{s}^{4}\right)$, Phys. Rev. Lett. 110 (2013) 252004, arXiv: 1303.6254 [hep-ph].

[83] M. Czakon, D. Heymes, and A. Mitov, High-precision differential predictions for top-quark pairs at the LHC, arXiv: 1511.00549 [hep-ph].

[84] P. Uwer, EasyNData: A Simple tool to extract numerical values from published plots, arXiv:0710.2896 [physics.comp-ph]. 